\title{
A New Approach to the Nazarov Reaction via Sequential Electrocyclic Ring Opening and Ring Closure
}

\author{
Tina N. Grant and F. G. West* \\ Department of Chemistry, University of Alberta, Edmonton, Alta., Canada T6G $2 G 2$.
}

Supporting Information: Experimental procedures, physical data and NMR spectra for 5ah, 6a-e, g, h, 7f, 8b-e, g, 9a, b, g, 10h, and synthetic intermediates (57 pages).

General Information. Reactions were carried out in flame-dried glassware under a positive nitrogen atmosphere unless otherwise stated. Transfer of anhydrous solvents and reagents was accomplished with oven-dried syringes or cannulae. Solvents were distilled before use: methylene chloride from calcium hydride, tetrahydrofuran, diethylether and benzene from sodium/benzophenone ketyl, toluene from sodium metal. Thin layer chromatography was performed on glass plates precoated with $0.25 \mathrm{~mm}$ Kieselgel $60 \mathrm{~F}_{254}$ (Merck). Flash chromatography column were packed with 230-400 mesh silica gel (Silicycle). Proton nuclear magnetic resonance spectra $\left({ }^{1} \mathrm{H}\right.$ NMR) were recorded at $400 \mathrm{MHz}$ or $500 \mathrm{MHz}$ and coupling constants $(J)$ are reported in Hertz $(\mathrm{Hz})$. Carbon nuclear magnetic resonance spectra $\left({ }^{13} \mathrm{C} \mathrm{NMR}\right)$ were recorded at $100 \mathrm{MHz}$ or $125 \mathrm{MHz}$ and are reported (ppm) relative to the center line of the triplet from chloroform- $d(77.23 \mathrm{ppm})$. Infrared (IR) spectra were measured with a Mattson Galaxy Series FT-IR 3000 spectrophotometer. Mass spectra were determined on a PerSeptive Biosystems Mariner high-resolution electrospray positive ion mode spectrometer.

2-(Triisopropylsiloxy)-1,3-butadiene (5a). Methyl vinyl ketone $(0.41 \mathrm{~mL}, 5.0 \mathrm{mmol})$ was dissolved in anhydrous $\mathrm{CH}_{2} \mathrm{Cl}_{2}(40 \mathrm{~mL})$. The temperature of the solution was dropped to $78^{\circ} \mathrm{C}$. Freshly distilled 2,6-lutidine $(0.87 \mathrm{~mL}, 7.5 \mathrm{mmol})$ was added dropwise to the reaction mixture. Triisopropylsilyl trifluoromethanesulfonate $(1.5 \mathrm{~mL}, 5.5 \mathrm{mmol})$ was added dropwise and the solution was stirred at $-78^{\circ} \mathrm{C}$ for $4 \mathrm{~h}$. The reaction was allowed to warm to room temperature before being quenched with a mixture of triethylamine $(20 \mathrm{~mL})$ and $\mathrm{H}_{2} \mathrm{O}(20$ $\mathrm{mL})$. The aqueous layer was extracted with $\mathrm{CH}_{2} \mathrm{Cl}_{2}(20 \mathrm{~mL})$. The organic layer was washed with $\mathrm{H}_{2} \mathrm{O}(2 \times 20 \mathrm{~mL})$ and brine $(20 \mathrm{~mL})$ and dried $\left(\mathrm{Na}_{2} \mathrm{SO}_{4}\right)$. The solvent was removed and the crude oil purified by flash column chromatography (silica gel, hexanes:EtOAc:TEA 25:1:1) to yield 2-(triisopropylsiloxy)-1,3-butadiene (463 mg, $2.0 \mathrm{mmol}, 41 \%$ ) as a colorless oil: $\mathrm{R}_{f} 0.72$ (hexanes/EtOAc 8:1); IR (thin film) 2946, 2893, 2868, 1586, 1464, 1375, 1303, 1060, 1009, 883, $684 \mathrm{~cm}^{-1} ;{ }^{1} \mathrm{H}$ NMR $\left(500 \mathrm{MHz} \mathrm{CDCl}_{3}\right) \delta 6.23(\mathrm{dd}, J=17.2,10.8 \mathrm{~Hz}, 1 \mathrm{H})$ $5.63(\mathrm{dd}, J=17.2,2.4 \mathrm{~Hz}, 1 \mathrm{H}), 5.13$ (br d, $J=10.4 \mathrm{~Hz}, 1 \mathrm{H}), 4.38$ (s, 1H), 4.32 (s, 1H), 1.25$1.34(\mathrm{~m}, 3 \mathrm{H}), 1.16(\mathrm{~d}, J=7.0 \mathrm{~Hz}, 18 \mathrm{H}) ;{ }^{13} \mathrm{C} \mathrm{NMR}\left(125 \mathrm{MHz}, \mathrm{CDCl}_{3}\right) \delta 155.6,135.2,114.6$, 95.3, 18.3, 13.1; HRMS (EI, $\mathrm{M}^{+}$) for $\mathrm{C}_{13} \mathrm{H}_{26} \mathrm{OSi}$ calcd 226.1753, found: $\mathrm{m} / \mathrm{z} 226.1754$.

(3E)-4-Isopropyl-2-(triisopropylsiloxy)-1,3-butadiene (5b). 5-Methyl-(3E)-hexene-2-one $(0.26 \mathrm{~mL}, 2.0 \mathrm{mmol})$ was dissolved in anhydrous THF $(5.0 \mathrm{~mL})$. The temperature of the solution was dropped to $0^{\circ} \mathrm{C}$. Freshly distilled triethylamine $(0.70 \mathrm{~mL}, 5.0 \mathrm{mmol})$ was added dropwise to the reaction mixture. Triisopropylsilyl trifluoromethanesulfonate $(0.59 \mathrm{~mL}, 2.2$ mmol) was added dropwise and the solution was stirred at $0^{\circ} \mathrm{C}$ for $2 \mathrm{~h}$. The reaction was quenched with a mixture of triethylamine $(1 \mathrm{~mL})$, hexanes $(5 \mathrm{~mL})$, and saturated $\mathrm{NaHCO}_{3}$ 
solution $(5 \mathrm{~mL})$. The organic layer was washed with $\mathrm{H}_{2} \mathrm{O}(2 \times 5 \mathrm{~mL})$ and brine $(5 \mathrm{~mL})$ and dried $\left(\mathrm{MgSO}_{4}\right)$. The solvent was removed to yield (3E)-4-isopropyl-2-(triisopropylsiloxy)1,3-butadiene, 5b, (535 mg, $2.0 \mathrm{mmol}, 100 \%$ ) as a colorless oil: $\mathrm{R}_{f} 0.84$ (hexanes/EtOAc $8: 1$ ); IR (thin film) 2960, 2868, 1589, 1464, 1306, 1026, 883, $678 \mathrm{~cm}^{-1} ;{ }^{1} \mathrm{H} \mathrm{NMR} \mathrm{(500} \mathrm{MHz,}$ $\left.\mathrm{CDCl}_{3}\right) \delta 6.06(\mathrm{dd}, J=15.5,6.5 \mathrm{~Hz}, 1 \mathrm{H}), 5.82(\mathrm{dd}, J=15.5,1.0 \mathrm{~Hz}, 1 \mathrm{H}), 4.23(\mathrm{~s}, 1 \mathrm{H}), 4.19$ (s, $1 \mathrm{H}), 2.36$ (app. octet, $J=7.0 \mathrm{~Hz}, 1 \mathrm{H}), 1.20-1.28(\mathrm{~m}, 3 \mathrm{H}), 1.11(\mathrm{~d}, J=7.5 \mathrm{~Hz}, 18 \mathrm{H}), 1.02(\mathrm{~d}, J$ $=7.0 \mathrm{~Hz}, 6 \mathrm{H}) ;{ }^{13} \mathrm{C} \mathrm{NMR}\left(125 \mathrm{MHz}, \mathrm{CDCl}_{3}\right) \delta 155.6,138.5,124.9,93.1,30.5,22.2,18.0$, 12.8; HRMS (EI, $\mathrm{M}^{+}$) for $\mathrm{C}_{16} \mathrm{H}_{32} \mathrm{OSi}$ calcd 268.2222, found: $\mathrm{m} / \mathrm{z} 268.2219$.

(3E)-3-Methyl-4-phenyl-2-(triisopropylsiloxy)-1,3-butadiene (5c). $\alpha$-Methyl-transcinnamaldehyde $(1.4 \mathrm{~mL}, 10.0 \mathrm{mmol})$ was dissolved in freshly distilled THF $(25 \mathrm{~mL})$. The temperature of the reaction mixture was dropped to $-78^{\circ} \mathrm{C}$ before adding MeLi (1.6 M, 6.3 $\mathrm{mL}, 10 \mathrm{mmol}$ ) dropwise by syringe. The reaction mixture was stirred at low temperature for 3 $\mathrm{h}$ and then quenched by the addition of saturated $\mathrm{NH}_{4} \mathrm{Cl}$ solution. The organic layer was washed with $\mathrm{H}_{2} \mathrm{O}(20 \mathrm{~mL})$ and brine $(20 \mathrm{~mL})$. The aqueous layer was extracted with $\mathrm{Et}_{2} \mathrm{O}(2$ x $20 \mathrm{~mL})$ and the combined organic layers were dried $\left(\mathrm{MgSO}_{4}\right)$. The solvent was removed and the crude oil was purified by flash column chromatography (silica gel, hexanes:EtOAc 10:1) to yield 3-methyl-4-phenyl-(3E)-butene-2-ol (1.6 g, $9.8 \mathrm{mmol}, 98 \%)$ as a pale yellow oil: $\mathrm{R}_{f}$ 0.38 (hexanes/EtOAc 2:1); IR (thin film) 3347, 2974, 1947, 1806, 1600, 1443, 1074, $698 \mathrm{~cm}^{-1}$; ${ }^{1} \mathrm{H}$ NMR (500 MHz, $\mathrm{CDCl}_{3}$ ) $\delta 7.34$ (app. t, $\left.J=7.5 \mathrm{~Hz}, 2 \mathrm{H}\right), 7.28$ (app. d, $J=6.5 \mathrm{~Hz}, 2 \mathrm{H}$ ), 7.22 (app. t, $J=7.0 \mathrm{~Hz}, 1 \mathrm{H}), 6.53$ (br s, $1 \mathrm{H}), 4.39$ (q, 1H, $J=6.5 \mathrm{~Hz}$ ), 1.89 (d, 3H, $J=1.0$ $\mathrm{Hz}), 1.60($ br s, $1 \mathrm{H}), 1.38(\mathrm{~d}, 3 \mathrm{H}, J=6.5 \mathrm{~Hz}) ;{ }^{13} \mathrm{C} \mathrm{NMR}\left(125 \mathrm{MHz}, \mathrm{CDCl}_{3}\right) \delta 141.6,137.6$, 128.9, 128.1, 126.4, 124.4, 73.6, 21.8, 13.4; HRMS (EI, $\left.\mathrm{M}^{+}\right)$for $\mathrm{C}_{11} \mathrm{H}_{14} \mathrm{O}$ calcd 162.1045, found: $\mathrm{m} / \mathrm{z} 162.1045$.

3-Methyl-4-phenyl-(3E)-butene-2-ol (1.56 g, $9.6 \mathrm{mmol})$ was dissolved in freshly distilled $\mathrm{CH}_{2} \mathrm{Cl}_{2}(30 \mathrm{~mL})$. To the solution was added powdered $4 \AA$ molecular sieves $(7 \mathrm{~g})$. The temperature of the reaction mixture was dropped to $0^{\circ} \mathrm{C}$ before adding NMO $(1.7 \mathrm{~g}, 14.4$ $\mathrm{mmol})$ in one portion. At low temperature, tetrapropylammonium perruthenate $(0.17 \mathrm{~g}, 0.48$ mmol) was next added in three equal portions. The reaction was left to stir at room temperature for $3 \mathrm{~h}$ before being quenched by filtration through a silica gel plug. The solvent was removed and the crude material purified by flash column chromatography (silica gel, hexanes:EtOAc 10:1) to yield 3-methyl-4-phenyl-(3E)-butene-2-one (1.3 g, $8.1 \mathrm{mmol}, 85 \%)$ as a white solid: m.p. 36-37.5 ${ }^{\circ} \mathrm{C}$; $\mathrm{R}_{f} 0.49$ (hexanes/EtOAc 2:1); IR (thin film) 3056, 2999, 2961, 2925, 1956, 1666, 1626, 1245, $1006 \mathrm{~cm}^{-1}$; ${ }^{1} \mathrm{H}$ NMR $\left(500 \mathrm{MHz}, \mathrm{CDCl}_{3}\right) \delta 7.52(\mathrm{~s}, 1 \mathrm{H})$, 7.42-7.45 (m, 4H), 7.34-7.38 (m, 1H), $2.48(\mathrm{~s}, 3 \mathrm{H}), 2.07$ (s, 3H); ${ }^{13} \mathrm{C} \mathrm{NMR}\left(125 \mathrm{MHz}, \mathrm{CDCl}_{3}\right)$ $\delta$ 200.3, 139.6, 137.8, 135.9, 129.7, 128.5, 128.4, 25.8, 12.9; HRMS (EI, $\mathrm{M}^{+}$) for $\mathrm{C}_{11} \mathrm{H}_{12} \mathrm{O}$ calcd 160.0888, found: $\mathrm{m} / \mathrm{z}$ 160.0884; Anal. Calcd for $\mathrm{C}_{11} \mathrm{H}_{12} \mathrm{O}: \mathrm{C}, 82.46$; H, 7.55. Found: C, $82.50 ; \mathrm{H}, 7.62$.

3-Methyl-4-phenyl-(3E)-butene-2-one $(0.20 \mathrm{~g}, 1.2 \mathrm{mmol})$ was dissolved in anhydrous THF $(3.5 \mathrm{~mL})$. The temperature of the solution was dropped to $0^{\circ} \mathrm{C}$. Freshly distilled triethylamine $(0.43 \mathrm{~mL}, 3.1 \mathrm{mmol})$ was added dropwise to the reaction mixture. Triisopropylsilyl trifluoromethanesulfonate $(0.40 \mathrm{~mL}, 1.5 \mathrm{mmol})$ was added dropwise and the solution was stirred at $0^{\circ} \mathrm{C}$ for $2 \mathrm{~h}$. The reaction was quenched with a mixture of triethylamine $(0.5 \mathrm{~mL})$, hexanes $(2.5 \mathrm{~mL})$, and saturated $\mathrm{NaHCO}_{3}$ solution $(5 \mathrm{~mL})$. The organic layer was washed with $\mathrm{H}_{2} \mathrm{O}(2 \times 5 \mathrm{~mL})$ and brine $(5 \mathrm{~mL})$ and dried $\left(\mathrm{MgSO}_{4}\right)$. The solvent was removed to yield (3E)-3-methyl-4-phenyl-2-(triisopropylsiloxy)-1,3-butadiene, 5c, (371 mg, 1.17 
mmol, 94\%) as a colorless oil: $\mathrm{R}_{f} 0.75$ (hexanes/EtOAc 8:1); IR (thin film) 3022, 2944, 2866, 1600, 1589, 1463, 1124, $1021 \mathrm{~cm}^{-1}$; ${ }^{1} \mathrm{H}$ NMR (500 MHz, $\left.\mathrm{CDCl}_{3}\right) \delta 7.35(\mathrm{t}, J=7.5 \mathrm{~Hz}, 2 \mathrm{H})$, $7.29(\mathrm{~d}, J=7.0 \mathrm{~Hz}, 2 \mathrm{H}), 7.23(\mathrm{t}, J=7.0 \mathrm{~Hz}, 1 \mathrm{H}), 7.18(\mathrm{br} \mathrm{s}, 1 \mathrm{H}), 4.59$ (d, $J=1.5 \mathrm{~Hz}, 1 \mathrm{H})$, $4.44(\mathrm{~d}, J=1.0 \mathrm{~Hz}, 1 \mathrm{H}), 2.00(\mathrm{~d}, J=1.0 \mathrm{~Hz}, 3 \mathrm{H}), 1.26-1.34(\mathrm{~m}, 3 \mathrm{H}), 1.15(\mathrm{~d}, J=7.5 \mathrm{~Hz}$, $18 \mathrm{H}) ;{ }^{13} \mathrm{C}$ NMR $\left(125 \mathrm{MHz}, \mathrm{CDCl}_{3}\right) \delta 157.8,138.2,133.0,129.3,128.0,127.1,126.4,92.0$, 18.1, 14.7, 12.9; HRMS (EI, $\mathrm{M}^{+}$) for $\mathrm{C}_{20} \mathrm{H}_{32} \mathrm{OSi}$ calcd, 316.2222, found: m/z 316.2218.

1-Triisopropylsiloxy-1-(cyclohexen-1-yl)-ethene (5d). 1-Acetyl-1-cyclohexene $(0.13 \mathrm{~mL}$, $1.0 \mathrm{mmol})$ was dissolved in anhydrous THF $(2.5 \mathrm{~mL})$. The temperature of the solution was dropped to $0^{\circ} \mathrm{C}$. Freshly distilled triethylamine $(0.35 \mathrm{~mL}, 2.5 \mathrm{mmol})$ was added dropwise to the reaction mixture. Triisopropylsilyl trifluoromethanesulfonate $(0.29 \mathrm{~mL}, 1.1 \mathrm{mmol})$ was added dropwise and the solution was stirred at $0^{\circ} \mathrm{C}$ for $2 \mathrm{~h}$. The reaction was quenched with a mixture of triethylamine $(0.5 \mathrm{~mL})$, hexanes $(2.5 \mathrm{~mL})$, and saturated $\mathrm{NaHCO}_{3}$ solution $(5 \mathrm{~mL})$. The organic layer was washed with $\mathrm{H}_{2} \mathrm{O}(2 \times 5 \mathrm{~mL})$ and brine $(5 \mathrm{~mL})$ and dried $\left(\mathrm{MgSO}_{4}\right)$. The solvent was removed to yield 1-(triisopropylsiloxy)-1-(cyclohexen-1-yl)-ethene, 5d, (278 $\mathrm{mg}, 0.99 \mathrm{mmol}, 99 \%$ ) as a colorless oil: $\mathrm{R}_{f} 0.76$ (hexanes/EtOAc 8:1); IR (thin film) 2943, 2867, 1591, 1464, 1288, 1017, $883 \mathrm{~cm}^{-1} ;{ }^{1} \mathrm{H}$ NMR (500 MHz, $\mathrm{CDCl}_{3}$ ) $\delta 6.32$ (br s, 1H), 4.31 $(\mathrm{s}, 1 \mathrm{H}), 4.18(\mathrm{~s}, 1 \mathrm{H}), 2.12-2.16(\mathrm{~m}, 4 \mathrm{H}), 1.64-1.70(\mathrm{~m}, 2 \mathrm{H}), 1.56-1.60(\mathrm{~m}, 2 \mathrm{H}), 1.20-1.28(\mathrm{~m}$, $3 \mathrm{H}), 1.11(\mathrm{~d}, J=7.0 \mathrm{~Hz}, 18 \mathrm{H}) ;{ }^{13} \mathrm{C}$ NMR $\left(125 \mathrm{MHz}, \mathrm{CDCl}_{3}\right) \delta 157.1,133.1,125.1,88.6,25.5$, 25.1, 22.8, 22.1, 18.1, 12.9; HRMS $\left(\mathrm{EI}, \mathrm{M}^{+}\right.$) for $\mathrm{C}_{17} \mathrm{H}_{32} \mathrm{OSi}$ calcd 280.2222, found: $\mathrm{m} / \mathrm{z}$ 280.2220 .

1-Triisopropylsiloxy-1-(cyclopenten-1-yl)-ethene (5e). The above procedure was used in the synthesis of 5e starting with 1-acetyl-1-cyclopentene $(0.11 \mathrm{~mL}, 1.0 \mathrm{mmol})$. The reaction yielded 1-(triisopropylsiloxy)-1-(cyclopenten-1-yl)-ethene, 5e, (266 mg, $1.0 \mathrm{mmol}, 100 \%)$ as a pale yellow oil: $\mathrm{R}_{f} 0.76$ (hexanes/EtOAc 8:1); IR (thin film) 2945, 2867, 1586, 1464, 1363, 1014, $883 \mathrm{~cm}^{-1}$; ${ }^{1} \mathrm{H}$ NMR $\left(500 \mathrm{MHz}, \mathrm{CDCl}_{3}\right.$ ) $\delta 6.06$ (br s, $\left.1 \mathrm{H}\right), 4.26(\mathrm{~s}, 1 \mathrm{H}), 4.24(\mathrm{~s}, 1 \mathrm{H}), 2.44$ (app. t, $J=7.5 \mathrm{~Hz}, 4 \mathrm{H}), 1.94$ (pent, $J=7.5 \mathrm{~Hz}, 2 \mathrm{H}), 1.20-1.28(\mathrm{~m}, 3 \mathrm{H}), 1.11(\mathrm{~d}, J=7.0 \mathrm{~Hz}$, $18 \mathrm{H}) ;{ }^{13} \mathrm{C} \mathrm{NMR}\left(125 \mathrm{MHz}, \mathrm{CDCl}_{3}\right) \delta 154.3,141.3,128.5,91.6,32.9,32.2,23.6,18.1,12.8$; HRMS (EI, $\mathrm{M}^{+}$) for $\mathrm{C}_{16} \mathrm{H}_{30}$ OSi calcd 266.2066, found: m/z 266.2067.

(1E,3Z)-1-Phenyl-3-(triisopropylsiloxy)-1,3-heptadiene (5f). N-morpholino cinnamide ${ }^{1}$ $(0.23 \mathrm{~g}, 1.0 \mathrm{mmol})$ was dissolved in freshly distilled THF $(10 \mathrm{~mL})$. The temperature of the reaction mixture was dropped to $-78^{\circ} \mathrm{C}$ before adding ${ }^{\mathrm{n}} \mathrm{BuLi}(1.6 \mathrm{M}$ in hexanes, $0.72 \mathrm{~mL}, 1.1$ $\mathrm{mmol}$ ) dropwise by syringe. The reaction mixture was stirred at low temperature for $3 \mathrm{~h}$ before being quenched by the addition of saturated $\mathrm{NH}_{4} \mathrm{Cl}$ solution. The organic layer was washed with $\mathrm{H}_{2} \mathrm{O}(10 \mathrm{~mL})$ and brine $(10 \mathrm{~mL})$. The aqueous layer was extracted with $\mathrm{Et}_{2} \mathrm{O}(2$ $\mathrm{x} 10 \mathrm{~mL})$ and the combined organic layers were dried $\left(\mathrm{MgSO}_{4}\right)$. The solvent was removed and the crude oil purified by flash column chromatography (silica gel, hexanes:EtOAc 8:1) to yield 1-phenyl-( $1 E)$-heptene-3-one $(0.053 \mathrm{~g}, 0.28 \mathrm{mmol}, 27 \%$ [unoptimized]) as a white solid: m.p. 34-36 ${ }^{\circ} \mathrm{C}$; $\mathrm{R}_{f} 0.48$ (hexanes/EtOAc 8:1); IR (thin film) 3023, 2954, 2939, 2864,

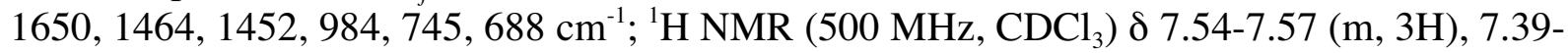
$7.41(\mathrm{~m}, 3 \mathrm{H}), 6.75(\mathrm{~d}, J=16.5 \mathrm{~Hz}, 1 \mathrm{H}), 2.67(\mathrm{t}, J=7.5 \mathrm{~Hz}, 2 \mathrm{H}), 1.67$ (pent, $J=7.5 \mathrm{~Hz}, 2 \mathrm{H}$ ), 1.39 (sextet, $J=7.5 \mathrm{~Hz}, 2 \mathrm{H}), 0.95(\mathrm{t}, J=7.0 \mathrm{~Hz}, 3 \mathrm{H}) ;{ }^{13} \mathrm{C} \mathrm{NMR}\left(125 \mathrm{MHz}, \mathrm{CDCl}_{3}\right) \delta 200.6$, 142.3, 134.6, 130.3, 128.9, 128.2, 126.3, 40.7, 26.5, 22.5, 13.9; HRMS (EI, $\mathrm{M}^{+}$) for $\mathrm{C}_{13} \mathrm{H}_{16} \mathrm{O}$ 
calcd 188.1201, found: $\mathrm{m} / \mathrm{z}$ 188.1201; Anal. Calcd for $\mathrm{C}_{13} \mathrm{H}_{16} \mathrm{O}: \mathrm{C}, 82.94 ; \mathrm{H}$, 8.57. Found: C, 82.74; H, 8.73.

1-Phenyl-(1E)-heptene-3-one $(0.086 \mathrm{~g}, 0.49 \mathrm{mmol})$ was dissolved in anhydrous THF $(1.5 \mathrm{~mL})$. The temperature of the solution was dropped to $0^{\circ} \mathrm{C}$. Freshly distilled triethylamine $(0.17 \mathrm{~mL}, 1.2 \mathrm{mmol})$ was added dropwise to the reaction mixture. Triisopropylsilyl trifluoromethanesulfonate $(0.17 \mathrm{~mL}, 0.62 \mathrm{mmol})$ was added dropwise and the solution was stirred at $0^{\circ} \mathrm{C}$ for $2 \mathrm{~h}$. The reaction was quenched with a mixture of triethylamine $(0.5 \mathrm{~mL})$, hexanes $(2.5 \mathrm{~mL})$, and saturated $\mathrm{NaHCO}_{3}$ solution $(5 \mathrm{~mL})$. The organic layer was washed with $\mathrm{H}_{2} \mathrm{O}(2 \times 5 \mathrm{~mL})$ and brine $(5 \mathrm{~mL})$ and dried $\left(\mathrm{MgSO}_{4}\right)$. The solvent was removed to yield (1E,3Z)-1-phenyl-3-(triisopropylsiloxy)-1,3-heptadiene (164 $\mathrm{mg}, 0.47 \mathrm{mmol}, 97 \%$ ) as a colorless oil: $\mathrm{R}_{f} 0.76$ (hexanes/EtOAc 8:1); IR (thin film) 3025, 2946, 2867, 1618, 1464, 1062 $\mathrm{cm}^{-1} ;{ }^{1} \mathrm{H}$ NMR $\left(500 \mathrm{MHz}, \mathrm{CDCl}_{3}\right) \delta 7.37$ (d, $\left.J=7.5 \mathrm{~Hz}, 2 \mathrm{H}\right), 7.31$ (app. t, $J=8.0 \mathrm{~Hz}, 2 \mathrm{H}$ ), $7.21(\mathrm{t}, J=7.5 \mathrm{~Hz}, 1 \mathrm{H}), 6.71(\mathrm{~d}, J=15.5 \mathrm{~Hz}, 1 \mathrm{H}), 6.56(\mathrm{~d}, J=15.5 \mathrm{~Hz}, 1 \mathrm{H}), 4.90(\mathrm{t}, J=7.5$ $\mathrm{Hz}, 1 \mathrm{H}), 2.18$ (q, $J=7.5 \mathrm{~Hz}, 2 \mathrm{H}), 1.42$ (sextet, $J=7.5 \mathrm{~Hz}, 2 \mathrm{H}), 1.22-1.30$ (m, 3H), 1.16 (d, $J$ $=7.0 \mathrm{~Hz}, 18 \mathrm{H}), 0.95(\mathrm{t}, J=7.5 \mathrm{~Hz}, 3 \mathrm{H}) ;{ }^{13} \mathrm{C} \mathrm{NMR}\left(125 \mathrm{MHz}, \mathrm{CDCl}_{3}\right) \delta 149.4,137.4,128.6$, 128.1, 127.1, 126.5, 126.3, 115.3, 28.3, 22.8, 18.1, 14.0, 13.9; HRMS (EI, $\mathrm{M}^{+}$) for $\mathrm{C}_{22} \mathrm{H}_{36} \mathrm{OSi}$ calcd, 344.2535, found: $\mathrm{m} / \mathrm{z} 344.2535$.

(1E,3Z)-2-Methyl-1-phenyl-3-(triisopropylsiloxy)-1,3-heptadiene (5g). $\alpha$-Methyl-transcinnamaldehyde $(1.4 \mathrm{~mL}, 10.0 \mathrm{mmol})$ was dissolved in freshly distilled $\mathrm{Et}_{2} \mathrm{O}(40 \mathrm{~mL})$. The temperature of the reaction mixture was dropped to $-78^{\circ} \mathrm{C}$ before adding ${ }^{n} \mathrm{BuLi}(1.45 \mathrm{M}$ in hexanes, $7.1 \mathrm{~mL}, 10.3 \mathrm{mmol}$ ) dropwise by syringe. The reaction mixture was stirred at low temperature for $4 \mathrm{~h}$ and then quenched by the addition of $0.5 \mathrm{M} \mathrm{HCl} \mathrm{(aq)} \mathrm{at} \mathrm{room} \mathrm{temperature.}$ The organic layer was washed with $\mathrm{H}_{2} \mathrm{O}(2 \times 30 \mathrm{~mL})$ and brine $(30 \mathrm{~mL})$. The aqueous layer was extracted with $\mathrm{Et}_{2} \mathrm{O}(2 \times 30 \mathrm{~mL})$ and the combined organic layers were dried $\left(\mathrm{MgSO}_{4}\right)$. The solvent was removed and the crude oil was purified by flash column chromatography (silica gel, hexanes:EtOAc 10:1) to yield 2-methyl-1-phenyl-(1E)-heptene-3-ol (1.3 g, 6.6 mmol, $66 \%$ ) as a colorless oil: $\mathrm{R}_{f} 0.24$ (hexanes/EtOAc 8:1); IR (thin film) 3349, 3024, 2956, 2860, 1446, $1011 \mathrm{~cm}^{-1}$; ${ }^{1} \mathrm{H}$ NMR $\left(500 \mathrm{MHz} \mathrm{CDCl}_{3}\right) \delta$ 7.18-7.37 (m, 5H), 6.49 (br s, $1 \mathrm{H}), 4.18(\mathrm{t}, J=6.6 \mathrm{~Hz}, 1 \mathrm{H}), 1.87(\mathrm{~d}, J=1.2 \mathrm{~Hz}, 3 \mathrm{H}), 1.65$ (app. q, $J=6.5 \mathrm{~Hz}, 2 \mathrm{H}), 1.59$ (br $\mathrm{s}, 1 \mathrm{H}), 1.24-1.44(\mathrm{~m}, 4 \mathrm{H}), 0.93(\mathrm{t}, J=7.2 \mathrm{~Hz}, 3 \mathrm{H}) ;{ }^{13} \mathrm{C} \mathrm{NMR}\left(125 \mathrm{MHz}, \mathrm{CDCl}_{3}\right) \delta 140.4$, 137.6, 128.9, 128.1, 126.4, 125.7, 78.2, 34.8, 28.0, 22.6, 14.0, 13.1; HRMS (EI, $\left.\mathrm{m}^{+}\right)$for $\mathrm{C}_{14} \mathrm{H}_{20} \mathrm{O}$ calcd 204.1514, found: $\mathrm{m} / \mathrm{z} 204.1510$.

2-Methyl-1-phenyl-(1E)-heptene-3-ol (2.1 g, $10.3 \mathrm{mmol})$ was dissolved in freshly distilled $\mathrm{CH}_{2} \mathrm{Cl}_{2}(30 \mathrm{~mL})$. To the solution was added powdered $4 \AA$ molecular sieves $(7 \mathrm{~g})$. The temperature of the reaction mixture was dropped to $0^{\circ} \mathrm{C}$ before adding NMO $(1.8 \mathrm{~g}, 31$ $\mathrm{mmol})$ in one portion. At low temperature, tetrapropylammonium perruthenate $(0.17 \mathrm{~g}, 0.50$ mmol) was next added in three equal portions. The reaction was left to stir at room temperature for $5 \mathrm{~h}$ before being quenched by filtration through a silica gel plug. The solvent was removed and the crude material purified by gradient column chromatography (silica gel, hexanes:EtOAc 50:1, 40:1, 30:1, 20:1) to yield (IE)-2-methyl-1-phenyl-heptene-3-one (1.6 g, $7.8 \mathrm{mmol}, 76 \%$ ) as a colorless oil: $\mathrm{R}_{f} 0.42$ (hexanes/EtOAc 8:1); IR (thin film) 3057, 2958, 2932, 2872, $1667 \mathrm{~cm}^{-1} ;{ }^{1} \mathrm{H}$ NMR $\left(300 \mathrm{MHz}, \mathrm{CDCl}_{3}\right) \delta 7.52$ (br q, $\left.J=1.2 \mathrm{~Hz}, 1 \mathrm{H}\right), 7.31-7.43$ $(\mathrm{m}, 5 \mathrm{H}), 2.80(\mathrm{t}, J=7.5 \mathrm{~Hz}, 2 \mathrm{H}), 2.07(\mathrm{~d}, J=1.5 \mathrm{~Hz}, 3 \mathrm{H}), 1.68$ (pent, $J=7.5 \mathrm{~Hz}, 2 \mathrm{H}), 1.40$ (sextet, $J=7.2 \mathrm{~Hz}, 2 \mathrm{H}), 0.96(\mathrm{t}, J=7.5 \mathrm{~Hz}, 3 \mathrm{H}) ;{ }^{13} \mathrm{C} \mathrm{NMR}\left(125 \mathrm{MHz}, \mathrm{CDCl}_{3}\right) \delta 202.7,138.3$, 
137.5, 136.1, 129.7, 128.4, 128.4, 37.4, 27.1, 22.5, 13.9, 13.2; HRMS (EI, $\mathrm{M}^{+}$) for $\mathrm{C}_{14} \mathrm{H}_{18} \mathrm{O}$ calcd 202.1358, found: $\mathrm{m} / \mathrm{z} 202.1363$.

(IE)-2-Methyl-1-phenyl-heptene-3-one $(0.20 \mathrm{~g}, 1.0 \mathrm{mmol})$ was dissolved in anhydrous THF $(2.5 \mathrm{~mL})$. The temperature of the solution was dropped to $0^{\circ} \mathrm{C}$. Freshly distilled triethylamine $(0.35 \mathrm{~mL}, 2.5 \mathrm{mmol})$ was added dropwise to the reaction mixture. Triisopropylsilyl trifluoromethanesulfonate $(0.32 \mathrm{~mL}, 1.2 \mathrm{mmol})$ was added dropwise and the solution was stirred at $0^{\circ} \mathrm{C}$ for $4 \mathrm{~h}$. The reaction was quenched with a mixture of triethylamine $(0.5 \mathrm{~mL})$, hexanes $(2.5 \mathrm{~mL})$, and saturated $\mathrm{NaHCO}_{3}$ solution $(5 \mathrm{~mL})$. The organic layer was washed with $\mathrm{H}_{2} \mathrm{O}(2 \times 5 \mathrm{~mL})$ and brine $(5 \mathrm{~mL})$ and dried $\left(\mathrm{MgSO}_{4}\right)$. The solvent was removed to yield (1E,3Z)-2-methyl-1-phenyl-3-(triisopropylsiloxy)-hepta-1,3-diene, 5g, (16:1, (IE,3Z):( $1 E, 3 E) ; 0.33 \mathrm{~g}, 0.93 \mathrm{mmol}, 93 \%$ ) as a pale yellow oil: $\mathrm{R}_{f} 0.75$ (hexanes/EtOAc 8:1); IR (thin film) 3022, 2958, 2867, 1622, 1464, $1090 \mathrm{~cm}^{-1}$; ${ }^{1} \mathrm{H}$ NMR $\left(500 \mathrm{MHz}, \mathrm{CDCl}_{3}\right) \delta 7.34(\mathrm{t}$, $J=7.5 \mathrm{~Hz}, 2 \mathrm{H}), 7.26-7.28(\mathrm{~m}, 2 \mathrm{H}), 7.22(\mathrm{t}, J=7.5 \mathrm{~Hz}, 1 \mathrm{H}), 6.86(\mathrm{~s}, 1 \mathrm{H}), 4.97(\mathrm{t}, J=7.0 \mathrm{~Hz}$, $1 \mathrm{H}), 2.19(\mathrm{q}, J=7.0 \mathrm{~Hz}, 2 \mathrm{H}), 1.99(\mathrm{~s}, 3 \mathrm{H}), 1.43$ (sextet, $J=7.5 \mathrm{~Hz}, 2 \mathrm{H}), 1.22-1.30(\mathrm{~m}, 3 \mathrm{H})$, $1.14(\mathrm{~d}, J=7.0 \mathrm{~Hz}, 18 \mathrm{H}), 0.96(\mathrm{t}, J=7.5 \mathrm{~Hz}, 3 \mathrm{H}) ;{ }^{13} \mathrm{C} \mathrm{NMR}\left(125 \mathrm{MHz}, \mathrm{CDCl}_{3}\right) \delta 152.0$, 138.4, 134.9, 129.1, 128.0, 126.2, 125.3, 111.1, 28.5, 23.0, 18.1, 15.5, 14.1, 13.9; HRMS (EI, $\mathrm{M}^{+}$) for $\mathrm{C}_{23} \mathrm{H}_{38} \mathrm{OSi}$ calcd, 358.2692, found: $\mathrm{m} / \mathrm{z} 358.2684$.

(3E)-2-(triisopropylsiloxy)-6-phenyl-1,3-hexadiene (5h). 1-(Triphenylphosphoranylidene)2-propanone $(0.32 \mathrm{~g}, 1.0 \mathrm{mmol})$ was dissolved in freshly distilled THF $(10 \mathrm{~mL})$. Hydrocinnamaldehyde $(0.13 \mathrm{~mL}, 1.0 \mathrm{mmol})$ was added dropwise to the reaction mixture at room temperature. The colorless solution was allowed to stir at room temperature for $18 \mathrm{~h}$ before being quenched by the addition of hexanes $(25 \mathrm{~mL})$. The addition of hexanes precipitated a white solid, which was removed by filtration. The solvent was removed and the crude oil was purified by flash column chromatography (silica gel, hexanes:EtOAc 15:1) to yield 6-phenyl-( $3 E)$-hexene-2-one $(0.09 \mathrm{~g}, 0.52 \mathrm{mmol}, 52 \%)$ as a colorless oil: $\mathrm{R}_{f} 0.22$ (hexanes/EtOAc 8:1); IR (thin film) 3028, 2928, 2858, 1697, 1675, 1627, 1360, 1255, 976, $748 \mathrm{~cm}^{-1}$; ${ }^{1} \mathrm{H}$ NMR $\left(400 \mathrm{MHz}, \mathrm{CDCl}_{3}\right) \delta$ 7.29-7.33 (m, 2H), 7.18-7.24 (m, 3H), 6.82 (dt, $J=$ $16.0,6.8 \mathrm{~Hz}, 1 \mathrm{H}), 6.10(\mathrm{dt}, J=16.0,1.6 \mathrm{~Hz}, 1 \mathrm{H}), 2.80(\mathrm{t}, J=7.6 \mathrm{~Hz}, 2 \mathrm{H}), 2.53-2.59(\mathrm{~m}, 2 \mathrm{H})$, 2.23 (s, 3H); ${ }^{13} \mathrm{C}$ NMR (125 MHz, $\left.\mathrm{CDCl}_{3}\right) \delta$ 198.5, 147.0, 140.6, 131.7, 128.5, 128.3, 126.2, 34.4, 34.1, 26.9; HRMS (EI, $\mathrm{M}^{+}$) for $\mathrm{C}_{12} \mathrm{H}_{14} \mathrm{O}$ calcd 174.1045, found: $\mathrm{m} / \mathrm{z}$ 174.1043.

6-Phenyl-( $3 E)$-hexene-2-one $(0.15 \mathrm{~g}, 0.86 \mathrm{mmol})$ was dissolved in anhydrous THF $(3.0 \mathrm{~mL})$. The temperature of the solution was dropped to $0^{\circ} \mathrm{C}$. Freshly distilled triethylamine $(0.30 \mathrm{~mL}, 2.1 \mathrm{mmol})$ was added dropwise to the reaction mixture. Triisopropylsilyl trifluoromethanesulfonate $(0.24 \mathrm{~mL}, 0.95 \mathrm{mmol})$ was added dropwise and the solution was stirred at $0^{\circ} \mathrm{C}$ for $2.5 \mathrm{~h}$. The reaction was quenched with a mixture of triethylamine $(0.5 \mathrm{~mL})$, hexanes $(2.5 \mathrm{~mL})$, and saturated $\mathrm{NaHCO}_{3}$ solution $(5 \mathrm{~mL})$. The organic layer was washed with $\mathrm{H}_{2} \mathrm{O}(2 \times 5 \mathrm{~mL})$ and brine $(5 \mathrm{~mL})$ and dried $\left(\mathrm{MgSO}_{4}\right)$. The solvent was removed to yield $(3 E)-$ 2-(triisopropylsiloxy)-6-phenyl-1,3-hexadiene, $\mathbf{5} \mathbf{h},(0.25 \mathrm{~g}, 0.84 \mathrm{mmol}, 98 \%)$ as a clear, colorless oil: $\mathrm{R}_{f} 0.82$ (hexanes:EtOAc 8:1); IR (thin film) 3028, 2944, 2867, 1589, 1464, 1321, 1028, 883, $697 \mathrm{~cm}^{-1}$; ${ }^{1} \mathrm{H}$ NMR (400 MHz, $\left.\mathrm{CDCl}_{3}\right) \delta$ 7.25-7.30 (m, 2H), 7.15-7.20 (m, $3 \mathrm{H}), 6.09(\mathrm{dt}, J=15.3,6.9 \mathrm{~Hz}, 1 \mathrm{H}), 5.90(\mathrm{dt}, J=15.3,1.2 \mathrm{~Hz}, 1 \mathrm{H}), 4.22(\mathrm{~s}, 1 \mathrm{H}), 4.17(\mathrm{~s}, 1 \mathrm{H})$, $2.73(\mathrm{t}, J=7.2 \mathrm{~Hz}, 2 \mathrm{H}), 2.42(\mathrm{dt}, J=7.5,7.5 \mathrm{~Hz}, 2 \mathrm{H}), 1.16-1.27(\mathrm{~m}, 3 \mathrm{H}), 1.10(\mathrm{~d}, J=6.6 \mathrm{~Hz}$, $18 \mathrm{H}) ;{ }^{13} \mathrm{C}$ NMR $\left(125 \mathrm{MHz}, \mathrm{CDCl}_{3}\right) \delta 155.3,141.8,130.5,128.5,128.4,128.3,125.8,93.2$, 35.7, 33.9, 18.1, 12.8; HRMS (EI, $\mathrm{M}^{+}$) for $\mathrm{C}_{21} \mathrm{H}_{34} \mathrm{OSi}$ calcd 330.2379, found: $\mathrm{m} / \mathrm{z} 330.2375$. 
1,1-Dichloro-2-(ethenyl)-2-triisopropylsilyloxycyclopropane (6a). Siloxy diene, 5a, (0.46 $\mathrm{g}, 2.0 \mathrm{mmol})$ was dissolved in $\mathrm{CHCl}_{3}(0.2 \mathrm{~mol}, 16 \mathrm{~mL})$. Benzyltriethylammonium chloride $(0.13 \mathrm{~g}, 0.60 \mathrm{mmol})$ was added to the reaction mixture. A solution of $50 \% \mathrm{NaOH}$ aq. $(0.37$ mol, $19.5 \mathrm{~mL}$ ) was then added in one portion and the reaction was vigorously stirred at room temperature for $4 \mathrm{~h}$. The reaction was quenched by dilution with $\mathrm{CH}_{2} \mathrm{Cl}_{2}(20 \mathrm{~mL})$ and $\mathrm{H}_{2} \mathrm{O}$ $(20 \mathrm{~mL})$. The organic layer was washed with $\mathrm{H}_{2} \mathrm{O}(2 \times 20 \mathrm{~mL})$. The aqueous layer was extracted with $\mathrm{CH}_{2} \mathrm{Cl}_{2}(2 \times 20 \mathrm{~mL})$ and the combined organic layers were dried $\left(\mathrm{MgSO}_{4}\right)$. The solvent was removed and the crude oil was purified by flash column chromatography (silica gel, hexanes:EtOAc 50:1) to yield 1,1-dichloro-2-(ethenyl)-2-triisopropylsiloxycyclopropane $(0.57 \mathrm{~g}, 1.86 \mathrm{mmol}, 93 \%)$ as a pale yellow oil: $\mathrm{R}_{f} 0.69$ (hexanes/EtOAc 10:1); IR (thin film) 2946, 2893, 2868, 1464, 1228, 1070, 883, $682 \mathrm{~cm}^{-1}$; ${ }^{1} \mathrm{H}$ NMR (500 MHz, $\mathrm{CDCl}_{3}$ ) $\delta 6.09$ (ddd, $J=1.0,10.5,17.0 \mathrm{~Hz}, 1 \mathrm{H}), 5.37(\mathrm{~d}, J=10.5 \mathrm{~Hz}, 1 \mathrm{H}), 5.32(\mathrm{~d}, J=17.0 \mathrm{~Hz}, 1 \mathrm{H}), 1.88(\mathrm{~d}, J=$ $8.5 \mathrm{~Hz}, 1 \mathrm{H}), 1.69(\mathrm{dd}, J=1.0,8.5 \mathrm{~Hz}, 1 \mathrm{H}), 1.07-1.16(\mathrm{~m}, 21 \mathrm{H}) ;{ }^{13} \mathrm{C} \mathrm{NMR}\left(125 \mathrm{MHz}, \mathrm{CDCl}_{3}\right)$ $\delta 135.9,118.4,65.4,64.6,32.4,18.3,18.2,13.0$; HRMS (EI, [M-Cl] $]^{+}$) for $\mathrm{C}_{14} \mathrm{H}_{26} \mathrm{OSiCl}$ calcd 273.1441, found: $\mathrm{m} / \mathrm{z} 273.1445$.

1,1-Dichloro-2-(3-methyl-[1E]-buten-1-yl)-2-triisopropylsilyloxycyclopropane (6b). The aforementioned method was also employed in the synthesis of $\mathbf{6 b}$ starting with (3E)-4isopropyl-2-(triisopropylsiloxy)-1,3-butadiene $(0.23 \mathrm{~g}, 0.84 \mathrm{mmol})$. The reaction was quenched after $1 \mathrm{~h}$ and the crude material was purified by flash column chromatography (silica gel, hexanes:EtOAc 50:1) to yield 1,1-dichloro-2-(3-methyl-trans-buten-1-yl)-2triisopropylsilyloxycyclopropane $(0.28 \mathrm{~g}, 0.80 \mathrm{mmol}, 95 \%)$ as a colorless oil: $\mathrm{R}_{f} 0.78$ (hexanes/EtOAc 20:1); IR (thin film) 2961, 2868, 1668, 1464, 1219, 1080, 970, 883, 769, 683 $\mathrm{cm}^{-1} ;{ }^{1} \mathrm{H}$ NMR $\left(500 \mathrm{MHz}, \mathrm{CDCl}_{3}\right) \delta 5.72(\mathrm{~d}, J=15.5 \mathrm{~Hz}, 1 \mathrm{H}), 5.66(\mathrm{dd}, J=15.5,6.0 \mathrm{~Hz}, 1 \mathrm{H})$, 2.38 (app. octet, $J=6.5 \mathrm{~Hz}, 1 \mathrm{H}), 1.84(\mathrm{~d}, J=8.5 \mathrm{~Hz}, 1 \mathrm{H}), 1.61(\mathrm{dd}, J=8.5,1.0 \mathrm{~Hz}, 1 \mathrm{H})$, $1.04-1.14(\mathrm{~m}, 21 \mathrm{H}), 1.02(\mathrm{~d}, J=7.0 \mathrm{~Hz}, 3 \mathrm{H}), 1.01(\mathrm{~d}, J=6.5 \mathrm{~Hz}, 3 \mathrm{H}) ;{ }^{13} \mathrm{C} \mathrm{NMR}(125 \mathrm{MHz}$, $\left.\mathrm{CDCl}_{3}\right) \delta 141.9,124.7,65.8,64.3,32.3,31.1,22.2,22.0,18.3,18.2$, 13.0; HRMS (EI, [M$\mathrm{Cl}]^{+}$) for $\mathrm{C}_{17} \mathrm{H}_{32} \mathrm{ClOSi}$ calcd 315.1911, found: $\mathrm{m} / \mathrm{z} 315.1895$.

(1E)-1,1-Dichloro-2-(1-phenylpropen-2-yl)-2-triisopropylsilyloxycyclopropane (6c). The same method was employed in the synthesis of $\mathbf{6 c}$ starting with (1E)-2-methyl-1-phenyl-3(triisopropylsiloxy)-1,3-butadiene $(0.30 \mathrm{~g}, 0.95 \mathrm{mmol})$. The reaction was quenched after $3 \mathrm{~h}$ and the crude material was purified by flash column chromatography (silica gel, hexanes:EtOAc 50:1) to yield ( 1 E)-1,1-dichloro-2-(1-phenylpropen-2-yl)-2triisopropylsilyloxycyclopropane $(0.30 \mathrm{~g}, 0.76 \mathrm{mmol}, 80 \%)$ as a colorless oil: $\mathrm{R}_{f} 0.58$ (hexanes/EtOAc 20:1); IR (thin film) 3024, 2945, 2867, 1495, 1257, 1076, 700, $690 \mathrm{~cm}^{-1}$; ${ }^{1} \mathrm{H}$ NMR $\left(500 \mathrm{MHz} \mathrm{CDCl}_{3}\right) \delta 7.36(\mathrm{t}, J=7.5 \mathrm{~Hz}, 2 \mathrm{H}), 7.24-7.28(\mathrm{~m}, 3 \mathrm{H}), 6.49(\mathrm{~s}, 1 \mathrm{H}), 2.13(\mathrm{~s}$, $3 \mathrm{H}), 2.14(\mathrm{~d}, J=8.5 \mathrm{~Hz}, 1 \mathrm{H}), 1.78(\mathrm{~d}, J=8.5 \mathrm{~Hz}, 1 \mathrm{H}), 1.06-1.18(\mathrm{~m}, 21 \mathrm{H}) ;{ }^{13} \mathrm{C} \mathrm{NMR}(125$ $\left.\mathrm{MHz}, \mathrm{CDCl}_{3}\right) \delta 136.7,135.8,129.4,128.9,128.2$, 127.0, 68.7, 64.8, 32.0, 18.0, 18.0, 16.0, 12.8; HRMS (EI, $\mathrm{M}^{+}$) for $\mathrm{C}_{21} \mathrm{H}_{32} \mathrm{Cl}_{2} \mathrm{OSi}$ calcd 398.1599, found: $\mathrm{m} / \mathrm{z} 398.1600$.

1,1-Dichloro-2-(1-cyclohexenyl)-2-triisopropylsilyloxycyclopropane (6d). The same method was employed in the synthesis of $\mathbf{6 d}$ starting with 1-triisopropylsiloxy-1-(cyclohexen1 -yl)-ethene $(0.12 \mathrm{~g}, 0.45 \mathrm{mmol})$. The reaction was quenched after $20 \mathrm{~min}$. and the crude material was purified by flash column chromatography (silica gel, hexanes:EtOAc 50:1) to yield 1,1-dichloro-2-(1-cyclohexenyl)-2-triisopropylsilyloxycyclopropane (0.16 g, $0.43 \mathrm{mmol}$, 
$96 \%$ ) as a colorless oil: $\mathrm{R}_{f} 0.63$ (hexanes/EtOAc 20:1); IR (thin film) 2943, 2867, 1464, 1249, 1099, 1081, 1069, 883, $682 \mathrm{~cm}^{-1} ;{ }^{1} \mathrm{H} \mathrm{NMR}\left(400 \mathrm{MHz}, \mathrm{CDCl}_{3}\right) \delta 5.70$ (app. septet, $J=$ $2.0 \mathrm{~Hz}, 1 \mathrm{H}), 2.38-2.45(\mathrm{~m}, 1 \mathrm{H}), 2.11-2.19(\mathrm{~m}, 1 \mathrm{H}), 2.04-2.09(\mathrm{~m}, 2 \mathrm{H}), 1.92(\mathrm{~d}, J=8.0 \mathrm{~Hz}$, $1 \mathrm{H}), 1.64-1.71(\mathrm{~m}, 2 \mathrm{H}), 1.62(\mathrm{~d}, J=8.0 \mathrm{~Hz}, 1 \mathrm{H}), 1.56-1.64(\mathrm{~m}, 2 \mathrm{H}), 1.07-1.16(\mathrm{~m}, 21 \mathrm{H}) ;{ }^{13} \mathrm{C}$ NMR $\left(125 \mathrm{MHz}, \mathrm{CDCl}_{3}\right) \delta 135.8,127.4,67.3,65.1,31.5,26.0,25.3,22.6,22.3,18.2,18.2$, 13.0; HRMS (EI, $\mathrm{M}^{+}$) for $\mathrm{C}_{17} \mathrm{H}_{32} \mathrm{OSiCl}_{2}$ calcd 362.1599, found: $\mathrm{m} / \mathrm{z} 362.1599$.

1,1-Dichloro-2-(1-cyclopentenyl)-2-triisopropylsilyloxycyclopropane (6e). The same method was employed in the synthesis of $6 \mathbf{e}$ starting with 1-triisopropylsiloxy-1-(cyclopenten1 -yl)-ethene $(0.15 \mathrm{~g}, 0.58 \mathrm{mmol})$. The reaction was quenched after $20 \mathrm{~min}$. and the crude material was purified by flash column chromatography (silica gel, hexanes:EtOAc 50:1) to yield 1,1-dichloro-2-(1-cyclopentenyl)-2-triisopropylsilyloxycyclopropane $\left(\begin{array}{lll}0.20 & \mathrm{~g}, \quad 0.57\end{array}\right.$ mmol, $97 \%$ ) as a colorless oil: $\mathrm{R}_{f} 0.63$ (hexanes/EtOAc 20:1); IR (thin film) 2946, 2868, 1464, 1265, 1243, 1099, 1068, 883, $683 \mathrm{~cm}^{-1}$; ${ }^{1} \mathrm{H}$ NMR $\left(500 \mathrm{MHz}, \mathrm{CDCl}_{3}\right) \delta 5.68$ (app. pent., $J=2.0 \mathrm{~Hz}, 1 \mathrm{H}), 2.58-2.65(\mathrm{~m}, 1 \mathrm{H}), 2.42-2.48(\mathrm{~m}, 1 \mathrm{H}), 2.34-2.39(\mathrm{~m}, 2 \mathrm{H}), 1.88-2.00(\mathrm{~m}, 2 \mathrm{H})$, $1.92(\mathrm{~d}, J=8.0 \mathrm{~Hz}, 1 \mathrm{H}), 1.70(\mathrm{~d}, J=8.0 \mathrm{~Hz}, 1 \mathrm{H}), 1.05-1.15(\mathrm{~m}, 21 \mathrm{H}) ;{ }^{13} \mathrm{C} \mathrm{NMR}(125 \mathrm{MHz}$, $\left.\mathrm{CDCl}_{3}\right) \delta 142.6,131.0,65.3,63.0,33.0,32.8,32.4,23.9,18.2,18.2,13.0$; HRMS (EI, $[\mathrm{M}-\mathrm{Cl}]^{+}$) for $\mathrm{C}_{17} \mathrm{H}_{30} \mathrm{OSiCl}$ calcd 313.1754, found: $\mathrm{m} / \mathrm{z} 313.1755$.

cis-1,1-Dichloro-2-(1-phenyl-[1E]-propen-2-yl)-3-propyl-2-

triisopropylsilyloxycyclopropane $(\mathbf{6 g})$. The same method was employed in the synthesis of 6g starting with (1E,3Z)-1-phenyl-3-(triisopropylsiloxy)-1,3-heptadiene (0.10 g, $0.28 \mathrm{mmol})$. The reaction was quenched after $1.5 \mathrm{~h}$, and the crude material was purified by flash column chromatography (silica gel, hexanes:EtOAc 50:1) to yield (1E)-1,1-dichloro-2-(1-phenylpropen-2-yl)-3-propyl-2-triisoprolylsilyloxycyclopropane $(0.11 \mathrm{~g}, 0.24 \mathrm{mmol}, 87 \%)$ as a colorless oil: $\mathrm{R}_{f} 0.69$ (hexanes/EtOAc 25:1); IR (thin film) 3027, 2947, 2868, 1630, 1464, 1257, 884, $682 \mathrm{~cm}^{-1} ;{ }^{1} \mathrm{H}$ NMR $\left(500 \mathrm{MHz} \mathrm{CDCl}_{3}\right) \delta 7.36(\mathrm{t}, J=7.5 \mathrm{~Hz}, 2 \mathrm{H}), 7.25-7.29(\mathrm{~m}$, $3 \mathrm{H}), 6.61(\mathrm{~s}, 1 \mathrm{H}), 2.11(\mathrm{~d}, J=1.5 \mathrm{~Hz}, 3 \mathrm{H}), 1.86-1.88(\mathrm{~m}, 1 \mathrm{H}), 1.57-1.65(\mathrm{~m}, 4 \mathrm{H}), 1.08-1.14$ $(\mathrm{m}, 21 \mathrm{H}), 1.03(\mathrm{t}, J=7.0 \mathrm{~Hz}, 3 \mathrm{H}) ;{ }^{13} \mathrm{C} \mathrm{NMR}\left(125 \mathrm{MHz}, \mathrm{CDCl}_{3}\right) \delta 136.8,136.2,131.4,128.9$, 128.3, 127.0, 70.8, 69.2, 38.8, 26.8, 21.6, 18.3, 18.1, 16.8, 14.0, 13.9; HRMS (EI, $\left.\mathrm{M}^{+}\right)$for $\mathrm{C}_{24} \mathrm{H}_{38} \mathrm{Cl}_{2} \mathrm{OSi}$ calcd 440.2069, found: $\mathrm{m} / \mathrm{z} 440.2078$.

(1E)-1,1-Dichloro-2-(4-phenyl-1-butenyl)-2-triisopropylsilyloxycyclopropane $(6 \mathrm{~h})$. The same method was employed in the synthesis of $\mathbf{6 h}$ starting with (1E)-2-(triisopropylsiloxy)-6phenyl-1,3-hexadiene $(0.26 \mathrm{~g}, 0.80 \mathrm{mmol})$. The reaction was quenched after $2 \mathrm{~h}$ and the crude material was purified by flash column chromatography (silica gel, hexanes:EtOAc 50:1) to yield (IE)-1,1-dichloro-2-(4-phenyl-1-butenyl)-2-triisopropylsilyloxycyclopropane $(0.27 \mathrm{~g}$, $0.65 \mathrm{mmol}, 72 \%$ ) as a colorless oil: $\mathrm{R}_{f} 0.70$ (hexanes/EtOAc 20:1); IR (thin film) 3027, 2944, 2867, 1463, 1222, 1083, 883, 697, $683 \mathrm{~cm}^{-1} ;{ }^{1} \mathrm{H}$ NMR (500 MHz, $\left.\mathrm{CDCl}_{3}\right) \delta$ 7.27-7.30 $(\mathrm{m}, 2 \mathrm{H}), 7.17-7.21(\mathrm{~m}, 3 \mathrm{H}), 5.82(\mathrm{~d}, J=15.5 \mathrm{~Hz}, 1 \mathrm{H}), 5.75(\mathrm{dt}, J=15.0,6.5 \mathrm{~Hz}, 1 \mathrm{H}), 2.73(\mathrm{t}, J$ $=9.0 \mathrm{~Hz}, 2 \mathrm{H}), 1.46(\mathrm{dt}, J=7.0,7.0 \mathrm{~Hz}, 2 \mathrm{H}), 1.81(\mathrm{~d}, J=8.5 \mathrm{~Hz}, 1 \mathrm{H}), 1.61(\mathrm{~d}, J=8.5 \mathrm{~Hz}$, $1 \mathrm{H}), 1.04-1.10(\mathrm{~m}, 21 \mathrm{H}) ;{ }^{13} \mathrm{C} \mathrm{NMR}\left(125 \mathrm{MHz}, \mathrm{CDCl}_{3}\right) \delta 141.6,134.3,128.7,128.6,128.3$, 126.2, 65.7, 64.2, 35.4, 33.9, 32.5, 18.3, 18.2, 13.0; HRMS (EI, [M-Cl] $]^{+}$) for $\mathrm{C}_{22} \mathrm{H}_{34} \mathrm{OSiCl}$ calcd 377.2068, found: $\mathrm{m} / \mathrm{z} 377.2066$. 
1-Phenyl-4-chloro-octa-1,4-dien-3-one (7f). The 2-siloxy-diene, 5f, $(0.12 \mathrm{~g}, 0.35 \mathrm{mmol})$ was dissolved in anhydrous toluene $(0.1 \mathrm{M}, 3.5 \mathrm{~mL}) . \mathrm{CHCl}_{3}(0.11 \mathrm{~mL}, 1.43 \mathrm{mmol})$ was then added and the temperature was dropped to $0^{\circ} \mathrm{C}$. Potassium tert-butoxide $(0.16 \mathrm{~g}, 1.4 \mathrm{mmol})$ was added in portions at $0^{\circ} \mathrm{C}$. The reaction was allowed to stir at low temperature for $1 \mathrm{~h}$ before $\mathrm{CHCl}_{3}(0.057 \mathrm{~mL}, 0.71 \mathrm{mmol})$ and potassium tert-butoxide $(0.08 \mathrm{~g}, 0.70 \mathrm{mmol})$ were added once again. The reaction was quenched by pouring into ice water after $1 \mathrm{~h}$ stirring at room temperature. The aqueous layer was extracted with $\mathrm{CH}_{2} \mathrm{Cl}_{2}(2 \times 10 \mathrm{~mL})$. The organic layer was washed with $\mathrm{H}_{2} \mathrm{O}(2 \times 10 \mathrm{~mL})$ and dried $\left(\mathrm{MgSO}_{4}\right)$. The crude material was purified by flash column chromatography (silica gel, hexanes:EtOAc 10:1) to yield 1-phenyl-4-chloroocta-1,4-dien-3-one $(0.045 \mathrm{~g}, 0.19 \mathrm{mmol}, 55 \%)$ as a white solid: m.p. $69-70^{\circ} \mathrm{C} ; \mathrm{R}_{f} 0.40$ (hexanes/EtOAc 8:1); IR (thin film) 3028, 2962, 2932, 2873, 1666, 1617, 1576, 1330, $759 \mathrm{~cm}^{-}$ ${ }^{1}$; ${ }^{1} \mathrm{H}$ NMR $\left(300 \mathrm{MHz}, \mathrm{CDCl}_{3}\right) \delta 7.83(\mathrm{~d}, J=15.6 \mathrm{~Hz}, 1 \mathrm{H}), 7.66-7.69(\mathrm{~m}, 2 \mathrm{H}), 7.44-7.49(\mathrm{~m}$, $4 \mathrm{H}), 7.12(\mathrm{t}, J=7.2 \mathrm{~Hz}, 1 \mathrm{H}), 2.49(\mathrm{q}, J=7.2 \mathrm{~Hz}, 2 \mathrm{H}), 1.65$ (sextet, $J=7.5 \mathrm{~Hz}, 2 \mathrm{H}), 1.07$ (t, $J$ $=7.5 \mathrm{~Hz}, 3 \mathrm{H}) ;{ }^{13} \mathrm{C} \mathrm{NMR}\left(125 \mathrm{MHz}, \mathrm{CDCl}_{3}\right) \delta 184.1,145.3,141.0,134.6,134.0,130.7,128.9$, 128.5, 120.5, 31.7, 21.2, 13.9; HRMS (EI, $\mathrm{M}^{+}$) for $\mathrm{C}_{14} \mathrm{H}_{15} \mathrm{ClO}$ calcd, 234.0811, found: $\mathrm{m} / \mathrm{z}$ 234.0805; Anal. Calcd for $\mathrm{C}_{14} \mathrm{H}_{15} \mathrm{ClO}: \mathrm{C}, 71.64 ; \mathrm{H}, 6.44$. Found: C, 71.31; H, 6.49.

2-Chloro-cyclopentenone (9a). 1,1-Dichloro-2-(ethenyl)-2triisopropylsilyloxycyclopropane, $6 \mathbf{a},(0.10 \mathrm{~g}, 0.35 \mathrm{mmol})$ was dissolved in $\mathrm{CH}_{2} \mathrm{Cl}_{2}(0.05 \mathrm{M}$, $7.0 \mathrm{~mL}) . \mathrm{AgBF}_{4}(0.10 \mathrm{~g}, 0.52 \mathrm{mmol})$ was added in one portion and the reaction mixture stirred at room temperature. The reaction was quenched after $24 \mathrm{~h}$ stirring by filtration through a pad of celite and silica gel. This compound was only observed as the major product in a crude reaction mixture. The product was not isolated since it readily decomposed during attempted purification by silica gel or alumina chromatography. Its presence was implied by partial spectral data: $\mathrm{R}_{f} 0.12$ (hexanes/EtOAc 8:1); ${ }^{1} \mathrm{H}$ NMR $\left(500 \mathrm{MHz}, \mathrm{CDCl}_{3}\right) \delta 7.60$ (t, $J=$ $3.0 \mathrm{~Hz}, 1 \mathrm{H}), 2.68-2.71(\mathrm{~m}, 2 \mathrm{H}), 2.53-2.58(\mathrm{~m}, 2 \mathrm{H})$.

5-Chloro-3-isopropyl-cyclopentenone (8b) and 2-Chloro-4-isopropyl-cyclopentenone (9b). 1,1-Dichloro-2-(3-methyl-trans-buten-1-yl)-2-triisopropylsilyloxycyclopropane, $\mathbf{6 b}$, $(0.12 \mathrm{~g}, 0.33 \mathrm{mmol})$ was dissolved in $\mathrm{CH}_{2} \mathrm{Cl}_{2}(0.05 \mathrm{M}, 6.6 \mathrm{~mL}) . \mathrm{AgBF}_{4}(0.096 \mathrm{~g}, 0.50 \mathrm{mmol})$ was added in one portion and the reaction mixture stirred at room temperature. The reaction was quenched after $4 \mathrm{~h}$ stirring at room temperature, by filtration through a pad of celite and silica gel. The crude material was purified by gradient column chromatography (silica gel, hexanes:EtOAc 50:1, 30:1, 25:1, 15:1, 10:1) to yield 5-chloro-3-isopropyl-cyclopentenone, $\mathbf{8 b},(0.017 \mathrm{~g}, 0.11 \mathrm{mmol}, 33 \%)$, 2-chloro-4-isopropyl-cyclopentenone, 9b, (0.010 g, 0.06 mmol, $19 \%$ ) and small amounts of 2-hydroxy4-I-propylcyclopentenone ${ }^{3}$ (presumed to result from capture of the Nazarov 2-siloxycyclopentenyl cation by adventitious water).

5-Chloro-3-isopropyl-cyclopentenone (8b). This compound was isolated as a pale yellow oil: $\mathrm{R}_{f} 0.28$ (hexanes/EtOAc 8:1); IR (thin film) 2968, 2933, 2875, 1719, 1610, 1174, 878, $859,757 \mathrm{~cm}^{-1} ;{ }^{1} \mathrm{H}$ NMR $\left(500 \mathrm{MHz}, \mathrm{CDCl}_{3}\right) \delta 6.01(\mathrm{dd}, J=3.0,2.0 \mathrm{~Hz}, 1 \mathrm{H}), 4.28(\mathrm{dd}, J=7.0$, $3.0 \mathrm{~Hz}, 1 \mathrm{H}$ ), 3.22 (dddd, $J=19.0,7.0,2.0,1.0 \mathrm{~Hz}, 1 \mathrm{H}$ ), 2.78 (dddd, $J=19.0,2.5,2.0,1.0 \mathrm{~Hz}$, $1 \mathrm{H}), 2.65$ (sept., $J=7.0 \mathrm{~Hz}, 1 \mathrm{H}), 1.20(\mathrm{~d}, J=7.0 \mathrm{~Hz}, 6 \mathrm{H}) ;{ }^{13} \mathrm{C}$ NMR $\left(125 \mathrm{MHz}, \mathrm{CDCl}_{3}\right) \delta$ 202.3, 185.6, 125.5, 54.2, 40.7, 32.4, 20.7; HRMS (EI, $\mathrm{M}^{+}$) for $\mathrm{C}_{8} \mathrm{H}_{11} \mathrm{ClO}$ calcd 158.0498, found: $\mathrm{m} / \mathrm{z} 158.0500$. 
2-Chloro-4-isopropyl-cyclopentenone (9b). This compound was isolated as a colorless oil: $\mathrm{R}_{f} 0.39$ (hexanes/EtOAc 8:1); IR (thin film) 2962, 2930, 2873, 1725, 1625, 1597, 1466, 1296, $1273,959,884 \mathrm{~cm}^{-1}$; ${ }^{1} \mathrm{H}$ NMR $\left(500 \mathrm{MHz}, \mathrm{CDCl}_{3}\right) \delta 7.55(\mathrm{~d}, J=2.5 \mathrm{~Hz}, 1 \mathrm{H}), 2.76$ (dddd, $J=$ 6.5, 6.0, 2.0, 2.0 Hz, 1H), $2.60(\mathrm{dd}, J=19.0,6.5 \mathrm{~Hz}, 1 \mathrm{H}), 2.24(\mathrm{dd}, J=19.0,2.0 \mathrm{~Hz}, 1 \mathrm{H}), 1.79$ (app. octet, $J=7.0 \mathrm{~Hz}, 1 \mathrm{H}), 0.98(\mathrm{~d}, J=7.0 \mathrm{~Hz}, 3 \mathrm{H}), 0.96(\mathrm{~d}, J=6.5 \mathrm{~Hz}, 3 \mathrm{H}) ;{ }^{13} \mathrm{C}$ NMR $(125$ $\mathrm{MHz}, \mathrm{CDCl}_{3}$ ) $\delta$ 200.7, 159.9, 135.8, 44.9, 37.5, 31.8, 19.8, 19.7; HRMS (EI, $\mathrm{M}^{+}$) for $\mathrm{C}_{8} \mathrm{H}_{11} \mathrm{ClO}$ calcd 158.0498 , found: $\mathrm{m} / \mathrm{z} 158.0494$.

2-Hydroxy-4-isopropyl-2-cyclopentenone. This compound was isolated in variable low yields under the previously mentioned reaction conditions: $\mathrm{R}_{f} 0.14$ (hexanes/EtOAc 8:1); IR (thin film) 3351, 2960, 2931, 2873, 1699, 1652, 1396, 1240, 1199, $1105 \mathrm{~cm}^{-1} ;{ }^{1} \mathrm{H}$ NMR (500 $\mathrm{MHz}, \mathrm{CDCl}_{3}$ ) $\delta 6.53$ (d, $J=3.5 \mathrm{~Hz}, 1 \mathrm{H}$ ), 5.49 (br. s, $1 \mathrm{H}$ ), 2.63 (dddd, $J=6.0,6.0,3.0,1.5 \mathrm{~Hz}$, $1 \mathrm{H}), 2.51$ (dd, $J=19.5,6.0 \mathrm{~Hz}, 1 \mathrm{H}), 2.14$ (dd, $J=19.5,1.5 \mathrm{~Hz}, 1 \mathrm{H}$ ), 1.70 (app. octet, $J=6.5$ $\mathrm{Hz}, 1 \mathrm{H}), 0.95(\mathrm{~d}, J=6.5 \mathrm{~Hz}, 3 \mathrm{H}), 0.93(\mathrm{~d}, J=7.0 \mathrm{~Hz}, 3 \mathrm{H}) ;{ }^{13} \mathrm{C}$ NMR $\left(125 \mathrm{MHz}, \mathrm{CDCl}_{3}\right) \delta$ 203.4, 152.6, 132.0, 41.3, 36.7, 32.2, 19.8, 19.7; HRMS (EI, $\mathrm{M}^{+}$) for $\mathrm{C}_{8} \mathrm{H}_{12} \mathrm{O}_{2}$ calcd 140.0837, found: $\mathrm{m} / \mathrm{z} 140.0838$.

5-Chloro-2-methyl-3-phenyl-cyclopentenone (8c). The above procedure was used in the preparation of $\mathbf{8 ~ c}$ starting with (l E)-1,1-dichloro-2-(1-phenylpropen-2-yl)-2triisoprolylsilyloxycyclopropane, $\mathbf{6 c},(0.08 \mathrm{~g}, 0.20 \mathrm{mmol})$. The reaction was quenched after $48 \mathrm{~h}$ stirring at room temperature. The crude material was purified by gradient column chromatography (silica gel, hexanes:EtOAc 20:1, 15:1, 10:1, 8:1, 5:1) to yield 5-chloro-2methyl-3-phenyl-cyclopentenone $(0.04 \mathrm{~g}, 0.17 \mathrm{mmol}, 87 \%)$ as an off-white solid: m.p. 99$100^{\circ} \mathrm{C} ; \mathrm{R}_{f} 0.26$ (hexanes/EtOAc 8:1); IR (thin film) 3056, 2943, 1702, 1615, 1352, 762, 698 $\mathrm{cm}^{-1}$; ${ }^{1} \mathrm{H}$ NMR $\left(400 \mathrm{MHz}, \mathrm{CDCl}_{3}\right) \delta$ 7.47-7.55 (m, $\left.5 \mathrm{H}\right), 4.43(\mathrm{dd}, J=7.2,2.8 \mathrm{~Hz}, 1 \mathrm{H}), 3.51$ (ddq, $J=18.0,6.8,2.0 \mathrm{~Hz}, 1 \mathrm{H}), 3.14(\mathrm{ddq}, J=18.4,2.0,2.0 \mathrm{~Hz}, 1 \mathrm{H}), 2.05(\mathrm{t}, J=2.0 \mathrm{~Hz}, 3 \mathrm{H}$ ); ${ }^{13} \mathrm{C}$ NMR $\left(125 \mathrm{MHz}, \mathrm{CDCl}_{3}\right) \delta 202.5,163.8,135.3,134.7,130.6,129.1,128.0,53.2,40.7$, 10.6; HRMS (EI, $\mathrm{M}^{+}$) for $\mathrm{C}_{12} \mathrm{H}_{11} \mathrm{ClO}$ calcd 208.0469, found: $\mathrm{m} / \mathrm{z}$ 208.0467; Anal. Calcd for $\mathrm{C}_{12} \mathrm{H}_{11} \mathrm{ClO}: \mathrm{C}, 69.74 ; \mathrm{H}, 5.36$. Found: $\mathrm{C}, 69.75 ; \mathrm{H}, 5.50$.

$\Delta^{1,6}$-8-Chloro-bicyclo[4.3.0]nonen-7-one (8d). The above procedure was used in the preparation of $\mathbf{8 d}$ starting from 1,1-dichloro-2-(1-cyclohexenyl)-2triisopropylsilyloxycyclopropane, $\mathbf{6 d},(0.11 \mathrm{~g}, 0.29 \mathrm{mmol})$. The reaction was quenched after $30 \mathrm{~h}$ stirring at room temperature. The crude material was purified by gradient column chromatography (silica gel, hexanes:EtOAc 50:1, 30:1, 25:1, 20:1, 10:1) to yield $\Delta^{1,6}-8$ chloro-bicyclo[4.3.0]nonen-7-one $(0.022 \mathrm{~g}, 0.13 \mathrm{mmol}, 45 \%)$ as a colorless oil: $\mathrm{R}_{f} 0.13$ (hexanes/EtOAc 8:1); IR (thin film) 2933, 2863, 1713, 1643, 1399, 1279, $731 \mathrm{~cm}^{-1}$; ${ }^{1} \mathrm{H}$ NMR $\left(500 \mathrm{MHz}, \mathrm{CDCl}_{3}\right) \delta 4.26$ (dd, $J=7.0,2.5 \mathrm{~Hz}, 1 \mathrm{H}$ ), 3.12 (app. dd, $J=18.5,7.0 \mathrm{~Hz}, 1 \mathrm{H}$ ), 2.68 (app. d, $J=19.0 \mathrm{~Hz} 1 \mathrm{H}), 2.26-2.41(\mathrm{~m}, 2 \mathrm{H}), 2.14-2.24(\mathrm{~m}, 2 \mathrm{H}), 1.74-1.79(\mathrm{~m}, 2 \mathrm{H}), 1.66-1.72$ (m, 2H); ${ }^{13} \mathrm{C} \mathrm{NMR}\left(125 \mathrm{MHz}, \mathrm{CDCl}_{3}\right) \delta$ 203.1, 171.3, 137.2, 53.6, 41.6, 28.6, 22.1, 21.7, 20.4; HRMS (EI, $\mathrm{M}^{+}$) for $\mathrm{C}_{9} \mathrm{H}_{11} \mathrm{OCl}$ calcd 172.0469, found: $\mathrm{m} / \mathrm{z} 172.0466$.

$\Delta^{1,5}$-3-Chloro-bicyclo[3.3.0]octen-2-one (8e). The above procedure was used in the preparation of $\mathbf{8 e}$ starting from 1,1-dichloro-2-(1-cyclopentenyl)-2triisopropylsilyloxycyclopropane, $6 \mathbf{e},(0.12 \mathrm{~g}, 0.33 \mathrm{mmol})$. The reaction was quenched after $48 \mathrm{~h}$ stirring at room temperature. The crude material was purified by gradient column 
chromatography (silica gel, hexanes:EtOAc 50:1, 30:1, 25:1, 20:1, 10:1) to yield $\Delta^{1,5}-3$ chloro-bicyclo[3.3.0]octen-2-one $(0.038 \mathrm{~g}, 0.24 \mathrm{mmol}, 73 \%)$ as a colorless oil: $\mathrm{R}_{f} 0.11$ (hexanes/EtOAc 8:1); IR (thin film) 2925, 2855, 1709, 1631, 1387, $732 \mathrm{~cm}^{-1} ;{ }^{1} \mathrm{H}$ NMR (400 $\left.\mathrm{MHz}, \mathrm{CDCl}_{3}\right) \delta 5.54$ (dd, $J=6.5,2.5 \mathrm{~Hz}, 1 \mathrm{H}$ ), 3.20 (app. dd, $J=18.5,6.4 \mathrm{~Hz}, 1 \mathrm{H}$ ), 2.71 (app. $\mathrm{d}, J=19.0 \mathrm{~Hz}, 1 \mathrm{H}), 2.56-2.61(\mathrm{~m}, 2 \mathrm{H}), 2.43-2.48(\mathrm{~m}, 2 \mathrm{H}), 2.38$ (app. pent, $J=6.4 \mathrm{~Hz}, 2 \mathrm{H}$ ); ${ }^{13} \mathrm{C}$ NMR $\left(125 \mathrm{MHz}, \mathrm{CDCl}_{3}\right) \delta 195.9,184.1,146.7,59.3,37.8,32.5,27.3,25.2$; HRMS (EI, $\mathrm{M}^{+}$) for $\mathrm{C}_{8} \mathrm{H}_{9} \mathrm{OCl}$ calcd 156.0342, found: $\mathrm{m} / \mathrm{z} 156.0341$.

5-Chloro-2-methyl-3-phenyl-4-propyl-cyclopentenone $(8 \mathrm{~g})$ and 2-Chloro-5-methyl-4phenyl-3-propyl-cyclopentenone $(9 \mathrm{~g})$. The above procedure was used in the preparation of $\mathbf{8 g}$ and $\mathbf{9 g}$ starting from ( $1 E$ )-1,1-dichloro-2-(1-phenyl-propen-2-yl)-3-propyl-2triisopropylsilyloxycyclopropane, $\mathbf{6 g},(0.10 \mathrm{~g}, 0.23 \mathrm{mmol})$. The reaction was quenched after 1 $\mathrm{h}$ stirring at room temperature. Purification by gradient column chromatography (silica gel, hexanes:EtOAc 20:1, 15:1, 10:1, 8:1, 5:1) yielded $\mathbf{8 g}$ (cis) : 9g(cis) : $\mathbf{8 g}$ (trans) : 9g(trans) (7.7 $: 4.5: 3: 1,87 \%){ }^{3}$ Products $\mathbf{9 g}($ cis $)$ and $\mathbf{8 g}$ (trans) were inseparable by standard techniques.

Major product $\mathbf{8 g}($ cis $)$ could be converted to the more stable $\mathbf{8 g}$ (trans) by stirring in $\mathrm{Et}_{2} \mathrm{O}$ in the presence of DBU to give a 12:1 mixture in favor of the trans diastereomer.

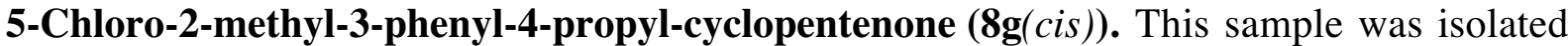
as a colorless oil: $\mathrm{R}_{f} 0.29$ (hexanes/EtOAc 8:1); IR (thin film) 3059, 2959, 2932, 2872, 1713, 1624, 1346, $699 \mathrm{~cm}^{-1} ;{ }^{1} \mathrm{H}$ NMR (500 MHz, $\left.\mathrm{CDCl}_{3}\right) \delta$ 7.44-7.50 (m, 3H), 7.34-7.40 (m, 2H), $4.64(\mathrm{~d}, J=6.5 \mathrm{~Hz}, 1 \mathrm{H}), 3.55-3.58(\mathrm{~m}, 1 \mathrm{H}), 1.92(\mathrm{~d}, J=2.0 \mathrm{~Hz}, 3 \mathrm{H}), 1.56-1.62(\mathrm{~m}, 2 \mathrm{H}), 1.19$ $1.26(\mathrm{~m}, 2 \mathrm{H}), 0.80(\mathrm{t}, J=7.0 \mathrm{~Hz}, 3 \mathrm{H}) ;{ }^{13} \mathrm{C} \mathrm{NMR}\left(125 \mathrm{MHz}, \mathrm{CDCl}_{3}\right) \delta 202.9,168.5,134.9$, 134.5, 129.5, 128.7, 127.4, 59.9, 45.3, 32.0, 20.1, 13.8, 9.8; HRMS (EI, $\mathrm{M}^{+}$) for $\mathrm{C}_{15} \mathrm{H}_{17} \mathrm{ClO}$ calcd 248.0968, found: $\mathrm{m} / \mathrm{z} 248.0968$.

2-Chloro-5-methyl-4-phenyl-3-propyl-cyclopentenone $(9 \mathrm{~g}($ cis $))$. The sample was contaminated with $(\mathbf{8 g}($ trans $))$, thus complete spectral data could not be reported: $\mathrm{R}_{f} 0.35$ (hexanes/EtOAc 8:1); Partial ${ }^{1} \mathrm{H}$ NMR (500 MHz, $\left.\mathrm{CDCl}_{3}\right) \delta 4.20(\mathrm{~d}, J=7.0 \mathrm{~Hz}, 1 \mathrm{H}), 2.86$ (pent, $J=7.5 \mathrm{~Hz}, 1 \mathrm{H}), 2.64$ (ddd, $J=14.0,9.5,7.0 \mathrm{~Hz}$ ), 2.13 (ddd, $J=14.5,9.5,5.5 \mathrm{~Hz}, 1 \mathrm{H}$ ), $1.39-1.56(\mathrm{~m}, 2 \mathrm{H}), 0.91(\mathrm{t}, J=7.5 \mathrm{~Hz}, 3 \mathrm{H}), 0.75(\mathrm{~d}, J=7.5 \mathrm{~Hz}, 3 \mathrm{H}) ;{ }^{13} \mathrm{C}$ NMR $(125 \mathrm{MHz}$, $\left.\mathrm{CDCl}_{3}\right) \delta 203.1,172.4,137.2,132.6,128.7,127.8,127.6,51.5,44.3,31.6,19.9,14.1,12.2$.

5-Chloro-2-methyl-3-phenyl-4-propyl-cyclopentenone $(\mathbf{8 g}($ trans $))$. The sample was contaminated with $(\mathbf{9 g}($ cis $))$, thus complete spectral data could not be reported: $\mathrm{R}_{f} 0.35$ (hexanes/EtOAc 8:1); ${ }^{1} \mathrm{H}$ NMR (500 MHz, $\left.\mathrm{CDCl}_{3}\right) \delta$ 7.43-7.51 (m, 3H), 7.35-7.37 (m, 2H), $4.05(\mathrm{~d}, J=2.5 \mathrm{~Hz}, 1 \mathrm{H}), 3.41$ (app. d, $J=10.0 \mathrm{~Hz} 1 \mathrm{H}), 1.91$ (d, $J=2.0 \mathrm{~Hz}, 3 \mathrm{H}), 1.66$ (dddd, $J$ $=14.0,10.5,6.5,3.0 \mathrm{~Hz}, 1 \mathrm{H}), 1.35-1.50(\mathrm{~m}, 2 \mathrm{H}), 1.20-1.30(\mathrm{~m}, 1 \mathrm{H}), 0.88(\mathrm{t}, J=7.0 \mathrm{~Hz}, 3 \mathrm{H})$; ${ }^{13} \mathrm{C}$ NMR $\left(125 \mathrm{MHz}, \mathrm{CDCl}_{3}\right) \delta$ 201.7, 169.3, 134.8, 134.4, 129.7, 128.7, 127.8, 58.6, 51.9, 34.6, 20.0, 13.9, 9.8 .

2-Chloro-5-methyl-4-phenyl-3-propyl-cyclopentenone (9g(trans)). This sample was isolated as a colorless oil: $\mathrm{R}_{f} 0.41$ (hexanes/EtOAc 8:1); IR (thin film) 3027, 2962, 2931, 2873, 1723, 1618, 1455, $703 \mathrm{~cm}^{-1}$; ${ }^{1} \mathrm{H}$ NMR (500 MHz, $\left.\mathrm{CDCl}_{3}\right) \delta$ 7.34-7.37 (m, 2H), 7.28$7.32(\mathrm{~m}, 1 \mathrm{H}), 7.11-7.13(\mathrm{~m}, 2 \mathrm{H}), 3.49(\mathrm{~d}, J=3.0 \mathrm{~Hz}, 3 \mathrm{H}), 2.58(\mathrm{ddd}, J=13.5,9.5,7.5 \mathrm{~Hz}$, 
$1 \mathrm{H}), 2.44(\mathrm{qd}, J=7.5,2.5 \mathrm{~Hz}, 1 \mathrm{H}), 2.07$ (ddd, $J=14.5,9.0,5.5 \mathrm{~Hz}, 1 \mathrm{H}), 1.49-1.56(\mathrm{~m}, 1 \mathrm{H})$, $1.38-1.44(\mathrm{~m}, 1 \mathrm{H}), 1.29(\mathrm{~d}, J=7.5 \mathrm{~Hz}, 3 \mathrm{H}), 0.89(\mathrm{t}, J=7.0 \mathrm{~Hz}, 3 \mathrm{H}) ;{ }^{13} \mathrm{C} \mathrm{NMR}(125 \mathrm{MHz}$, $\left.\mathrm{CDCl}_{3}\right) \delta 203.1,173.0,140.3,131.8,129.4,127.9,127.8,56.0,50.1,31.3,20.3,15.5,14.2$; HRMS (EI, $\mathrm{M}^{+}$) for $\mathrm{C}_{15} \mathrm{H}_{17} \mathrm{ClO}$ calcd 248.0968, found: $\mathrm{m} / \mathrm{z} 248.0973$.

2,3,4,5-Tetrahydrocyclopenta[ $[\boldsymbol{a}]$ napthalen-1-one (10h). The above procedure was used in the preparation of $\mathbf{1 0 h}$ from ( $1 E$ )-1,1-dichloro-2-(4-phenyl-1-buten-1-yl)-2triisopropylsilyloxycyclopropane, $6 \mathbf{h},(0.09 \mathrm{~g}, 0.22 \mathrm{mmol})$. The reaction was quenched after $18 \mathrm{~h}$ stirring at room temperature. The crude material was purified by gradient column chromatography (silica gel, hexanes:EtOAc 20:1, 15:1, 10:1, 8:1, 5:1) to yield 2,3,4,5tetrahydrocyclopenta $[a]$ napthalen-1-one $(0.028 \mathrm{~g}, 0.15 \mathrm{mmol}, 63 \%)$ as an off-white solid: m.p. 102-104 ${ }^{\circ}$; $\mathrm{R}_{f} 0.07$ (hexanes/EtOAc 8:1); IR (thin film) 3067, 2947, 2933, 2906, 2841, $1683,1629,1435,765 \mathrm{~cm}^{-1}$; ${ }^{1} \mathrm{H}$ NMR $\left(500 \mathrm{MHz}, \mathrm{CDCl}_{3}\right) \delta 8.24(\mathrm{~d}, J=7.5 \mathrm{~Hz}, 1 \mathrm{H}), 7.17-7.25$ $(\mathrm{m}, 3 \mathrm{H}), 2.96(\mathrm{t}, J=8.0 \mathrm{~Hz}, 2 \mathrm{H}), 2.68-2.70(\mathrm{~m}, 2 \mathrm{H}), 2.66(\mathrm{t}, J=8.0 \mathrm{~Hz}, 2 \mathrm{H}), 2.57-2.59(\mathrm{~m}$, $2 \mathrm{H}) ;{ }^{13} \mathrm{C}$ NMR $\left(125 \mathrm{MHz}, \mathrm{CDCl}_{3}\right) \delta$ 202.8, 174.8, 134.9, 134.4, 129.1, 127.8, 127.5, 126.7, 123.9, 35.9, 29.2, 27.6, 27.1; HRMS (EI, $\mathrm{M}^{+}$) for $\mathrm{C}_{13} \mathrm{H}_{12} \mathrm{O}$ calcd 184.0888, found: $\mathrm{m} / \mathrm{z}$ 184.0886. (Data are in accord with previously published literature values. ${ }^{4}$ )

\section{$\underline{\text { References }}$}

1. Badioli, M.; Ballini, R.; Bartolacci, M.; Bosica, G.; Torregiani, E.; Marcantoni, E. J. Org. Chem. 2002, 67, 8938-8942.

2. $\quad$ Carter, C. A. G.; Casty, G. L.; Stryker, J. M. Synlett 2001, 1046-1049.

3. Stereochemical assignments were based on analysis of $\mathrm{H}_{4}-\mathrm{H}_{5}$ coupling constants, in analogy to the work of Noyori et al.: Hayakawa, Y.; Yokoyama, K.; Noyori, R. J. Am. Chem. Soc. 1978, 100, 1799-1806.

4. Gagnier, S. V.; Larock, R. C. J. Am. Chem. Soc. 2003, 125, 4904-4807. 


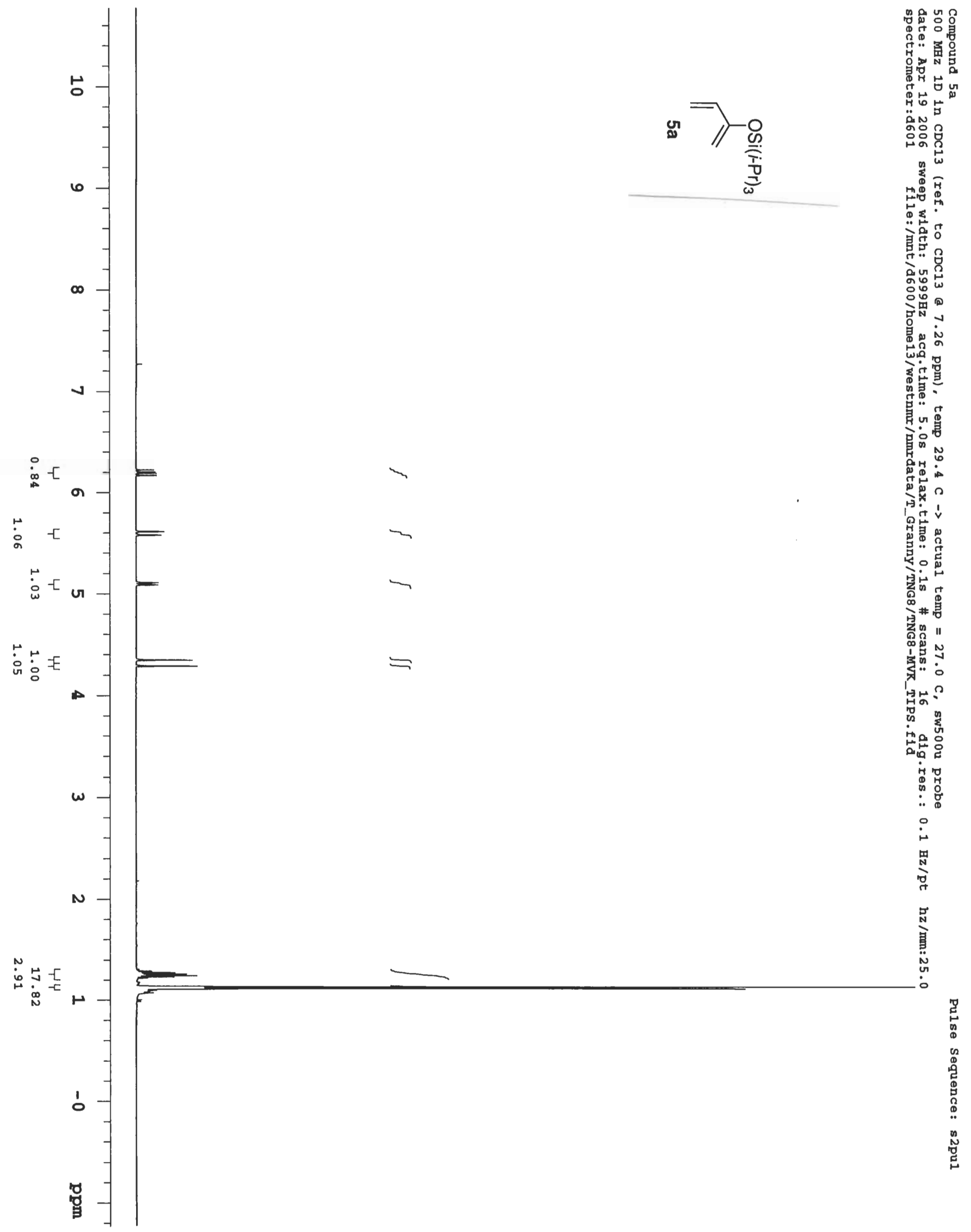




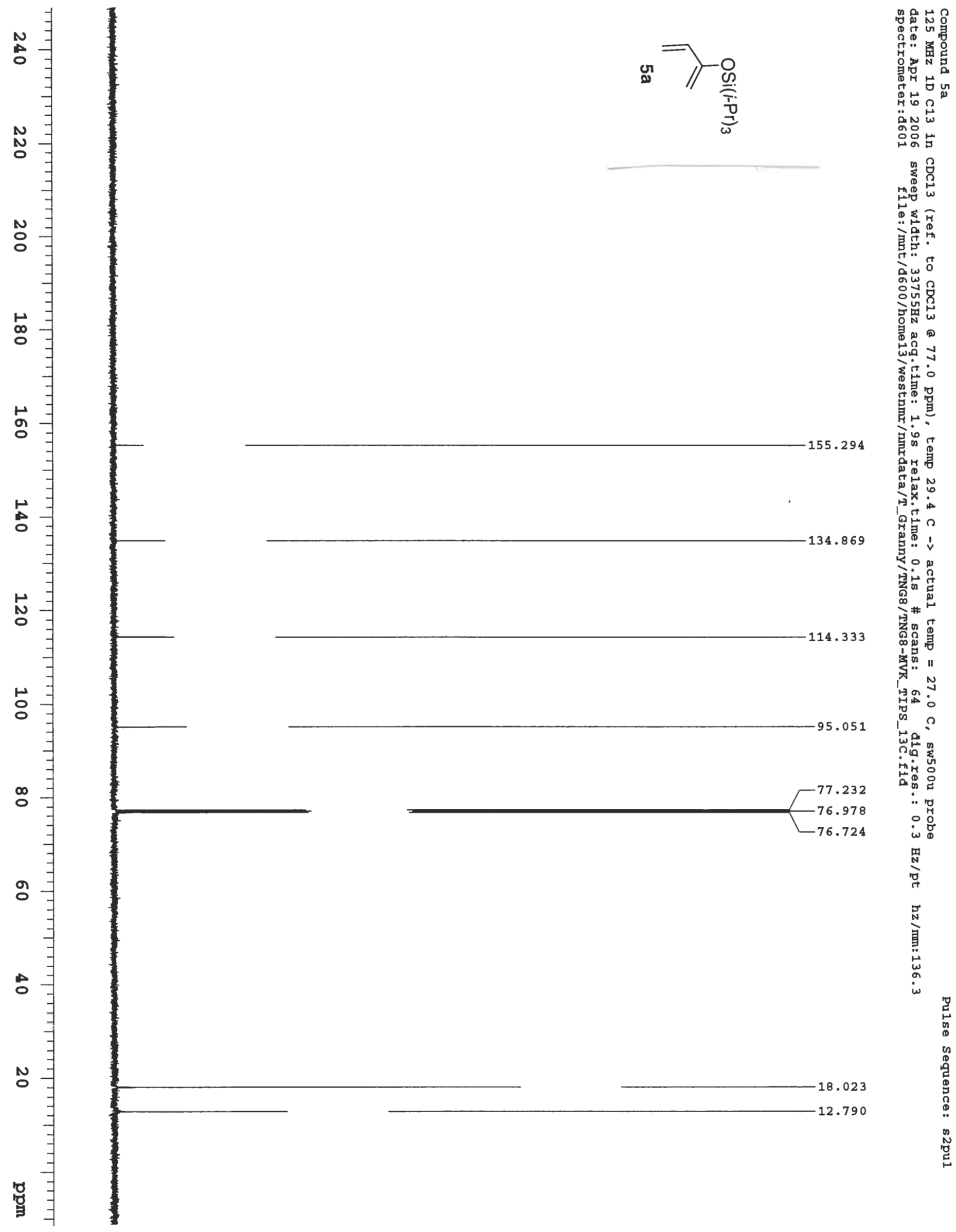



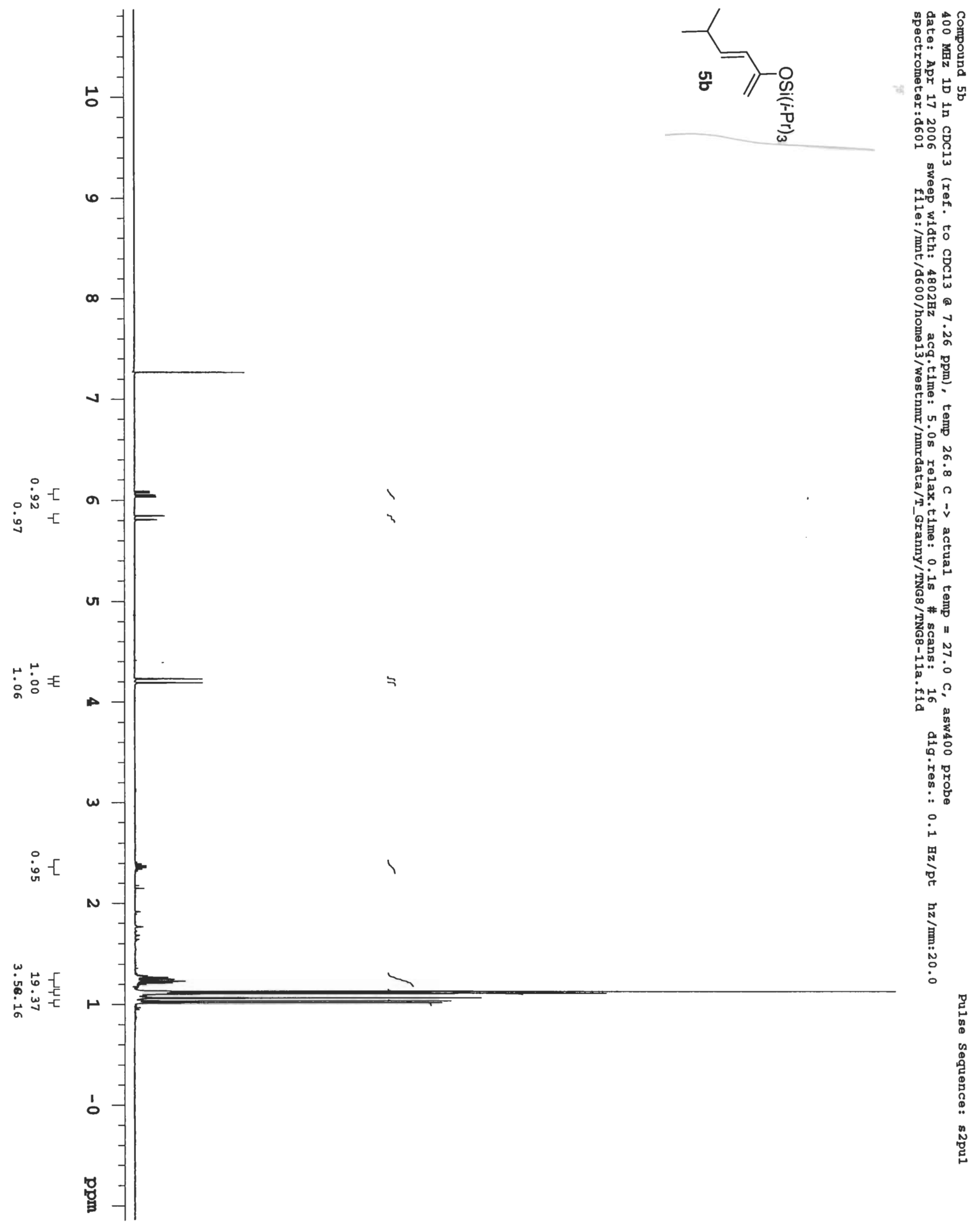

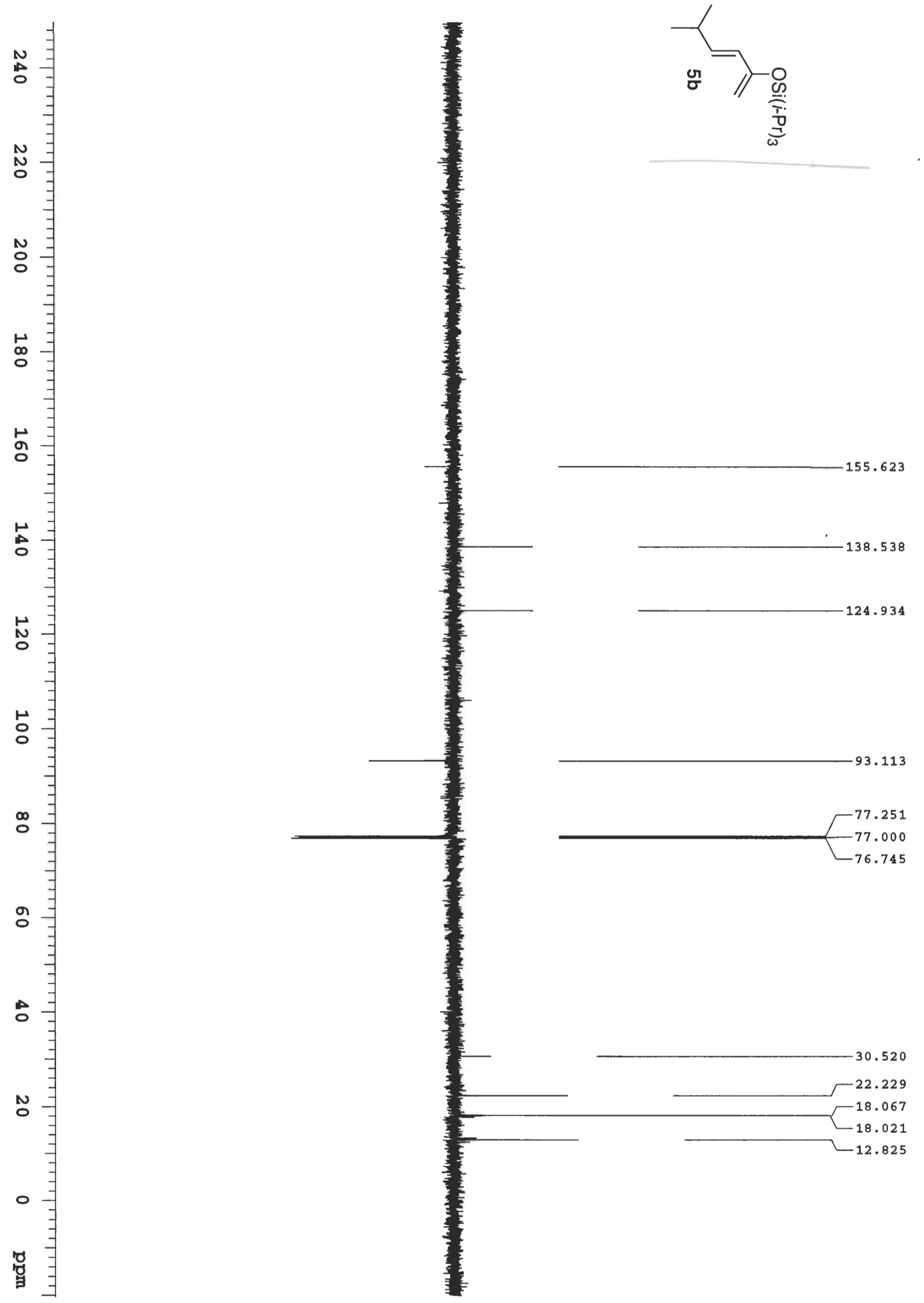

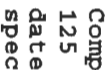

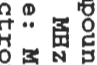

管

क

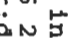

응

म

嵒 $\omega$

的要

क्ष?

官守

त.

ôw

象 $\omega$

引田 ه

건

要然:

玉े

on

政

毠

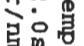

西

政

?

1

然

灵“踝

$-124.934$

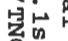

葛的

穴*

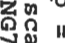

谓

ํํำ

No

苟?
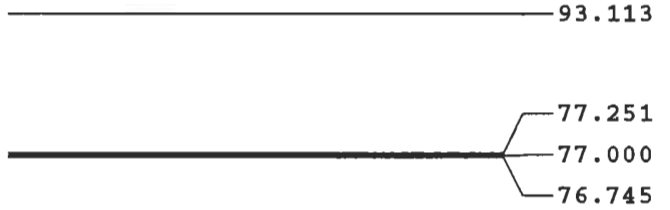

in

它乌

藏

i

思 


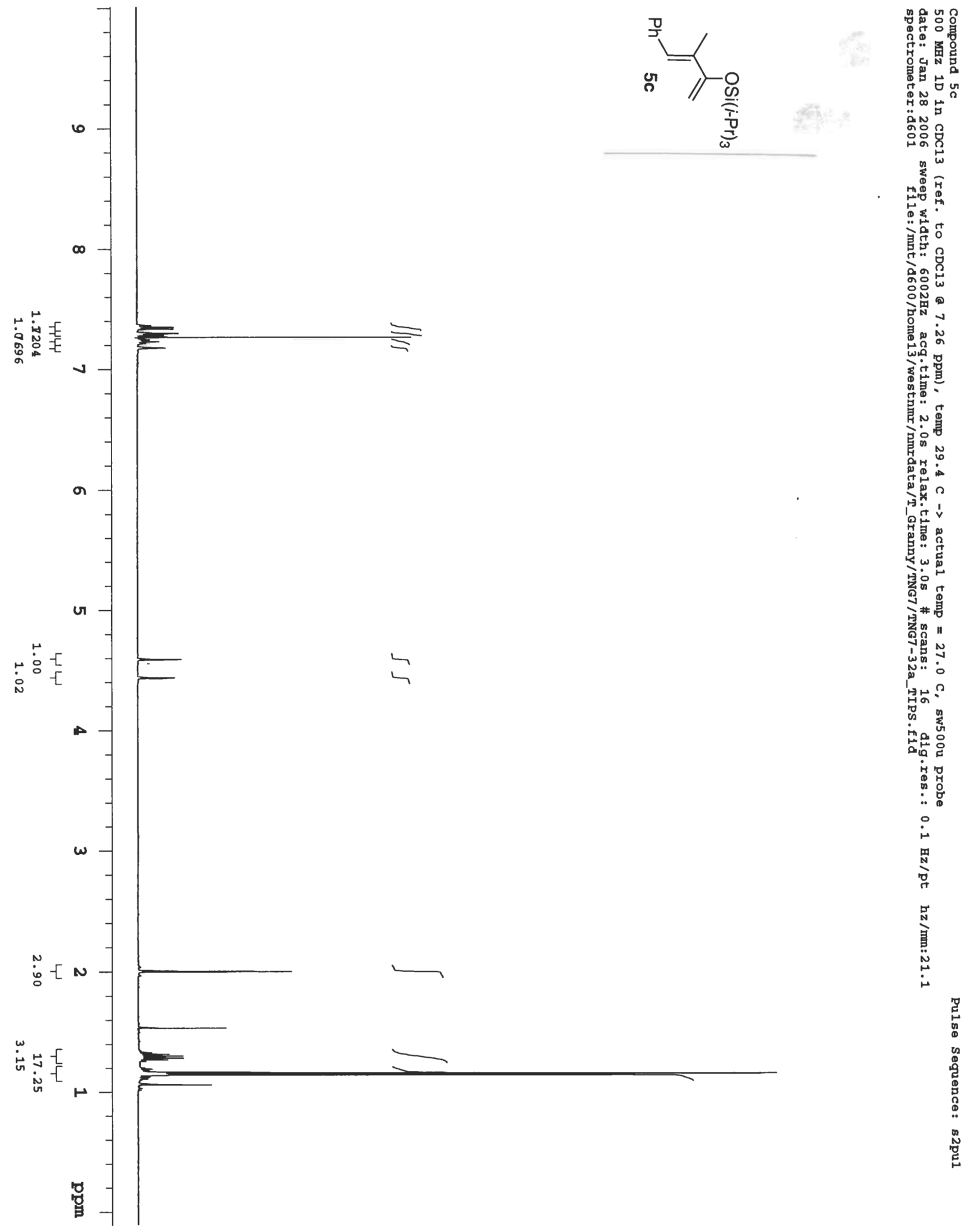




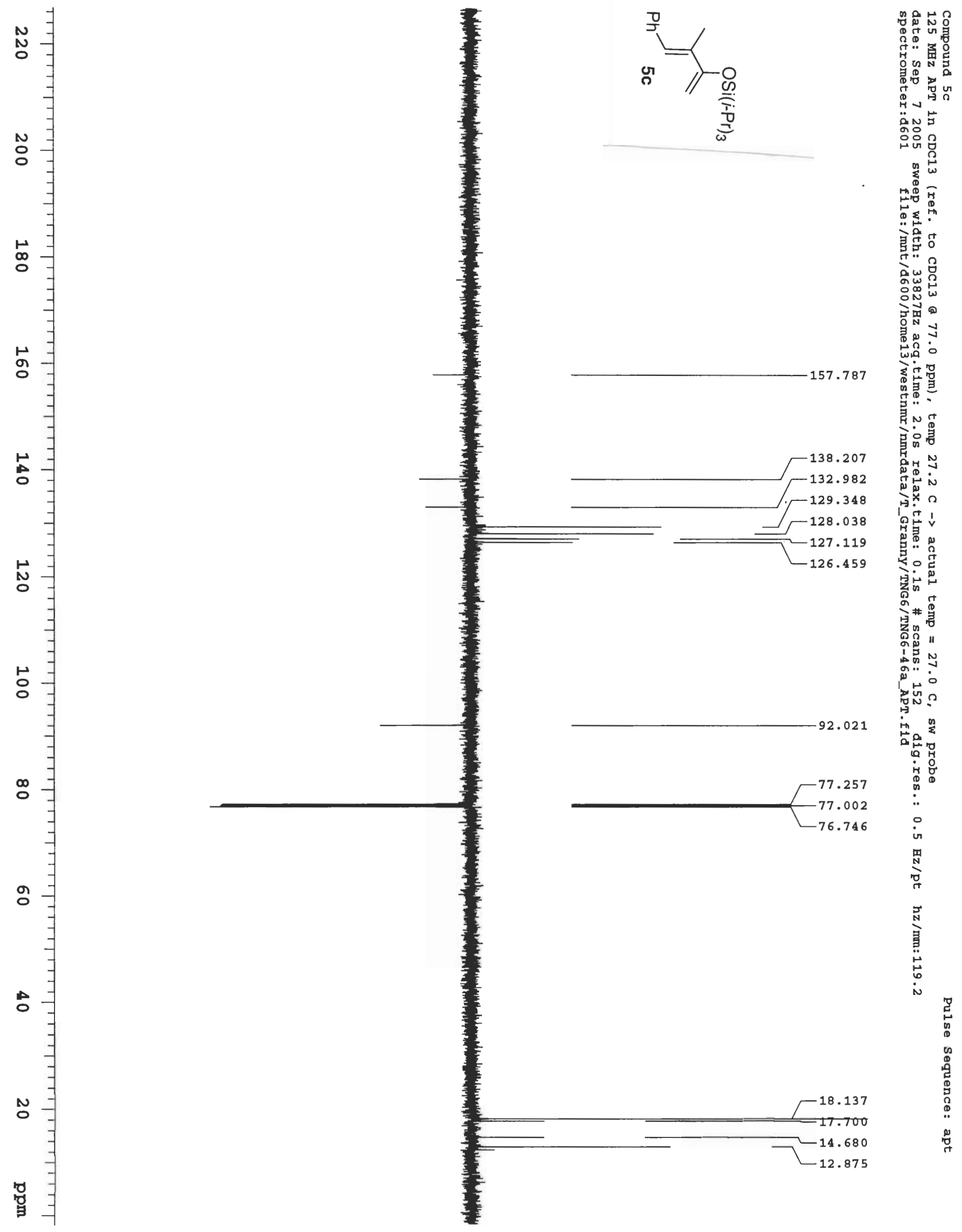




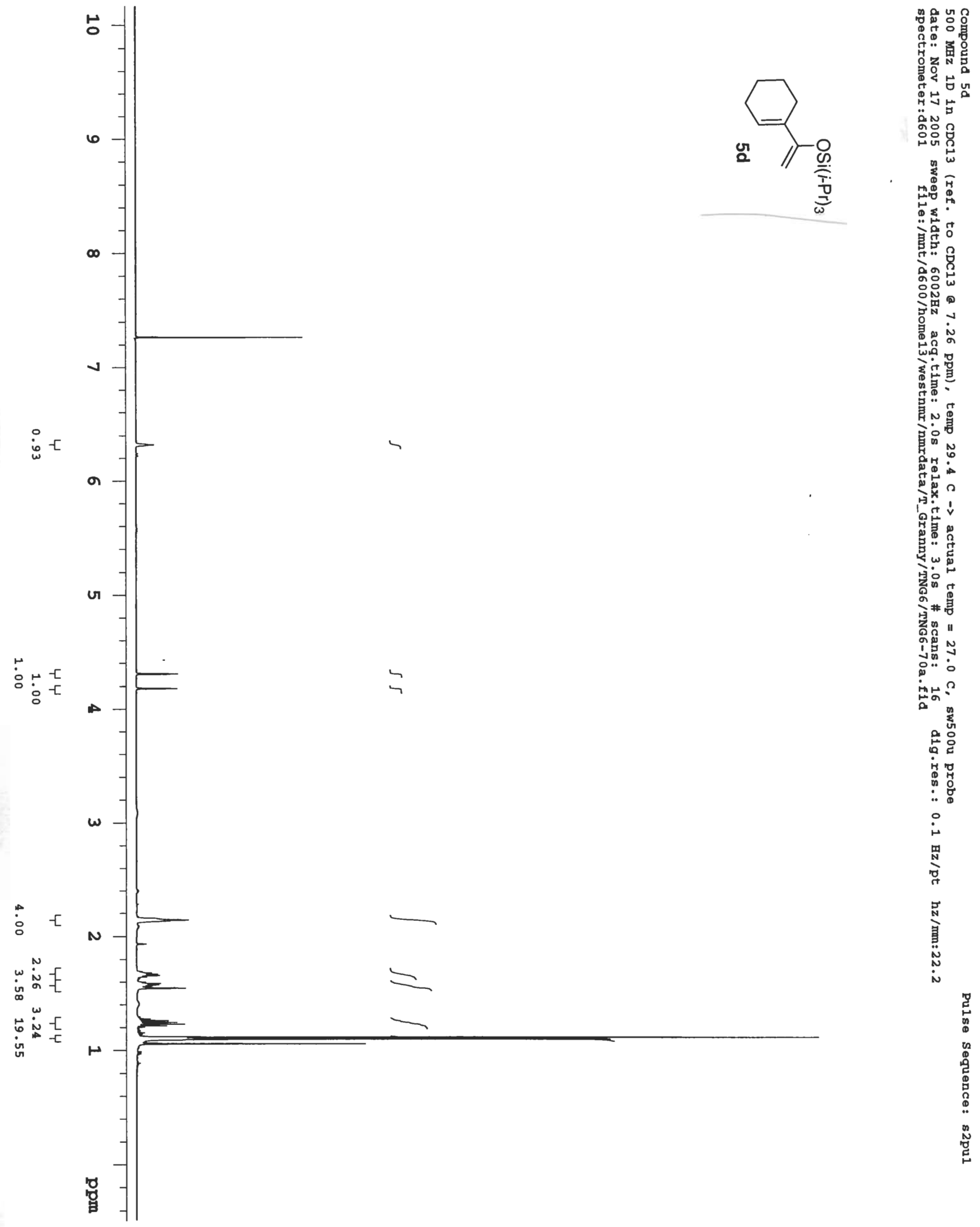




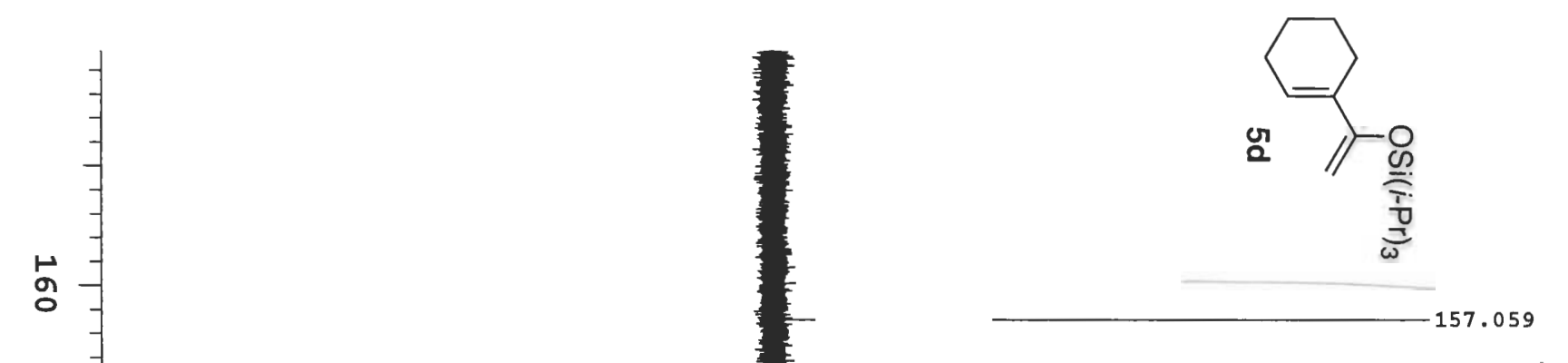

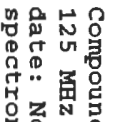

品然

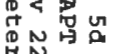

䒺占

ㅇํㅇㅇㅛ

哇

禺思

क्ष:

氛然

홍

133.156

空

วNํํํ

要

害思

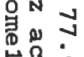

ผึ:

125.139

농

$-88.571$

$\stackrel{\infty}{\circ}$

77.246

$-76.991$

76.760

$-76.736$

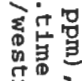

最,

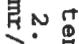

官品星

渵

2

它

웅

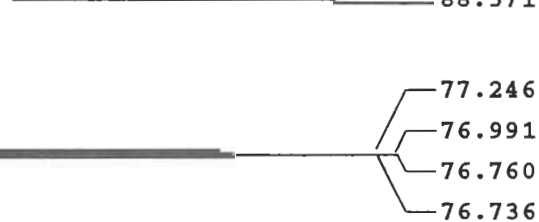

.

웅

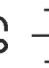

(

$\therefore$

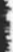

蛋。虽

案品

范

雾琞

ลूก "

正

"ूo

密品?

in

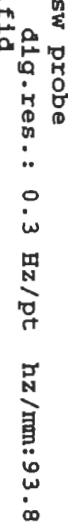

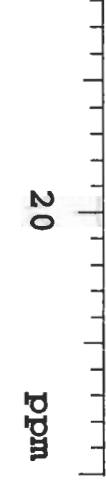

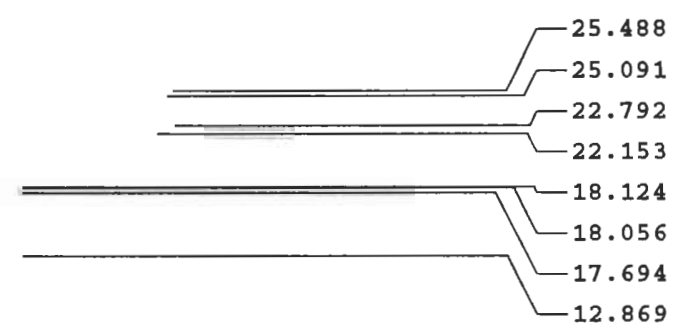

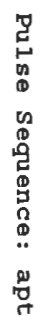

S-19 


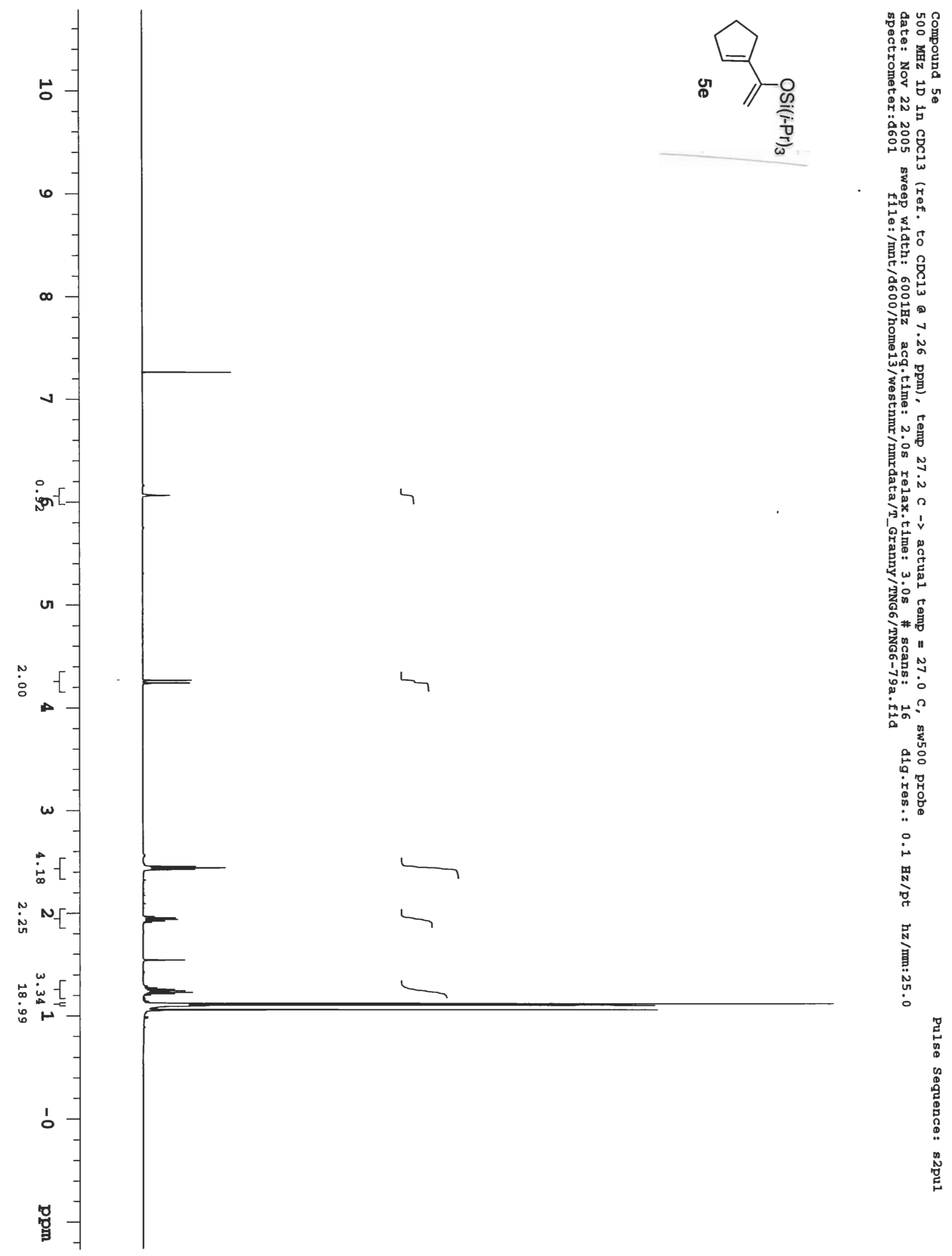




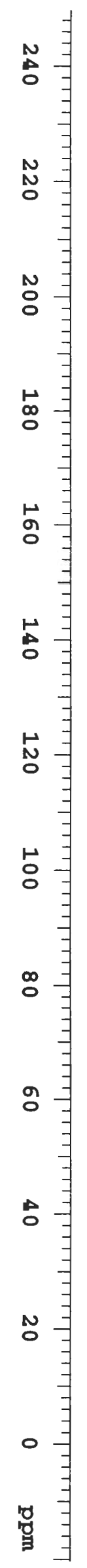

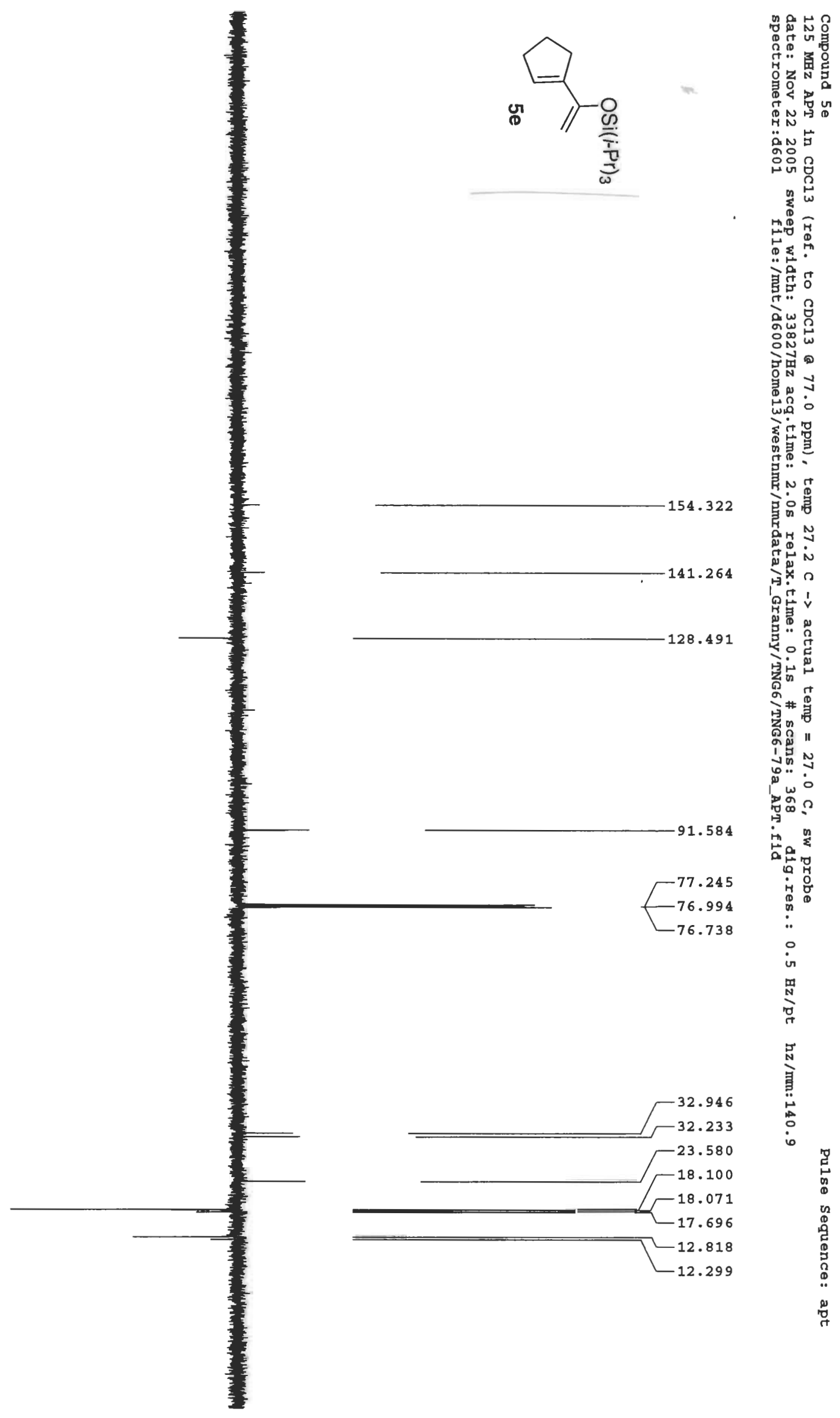




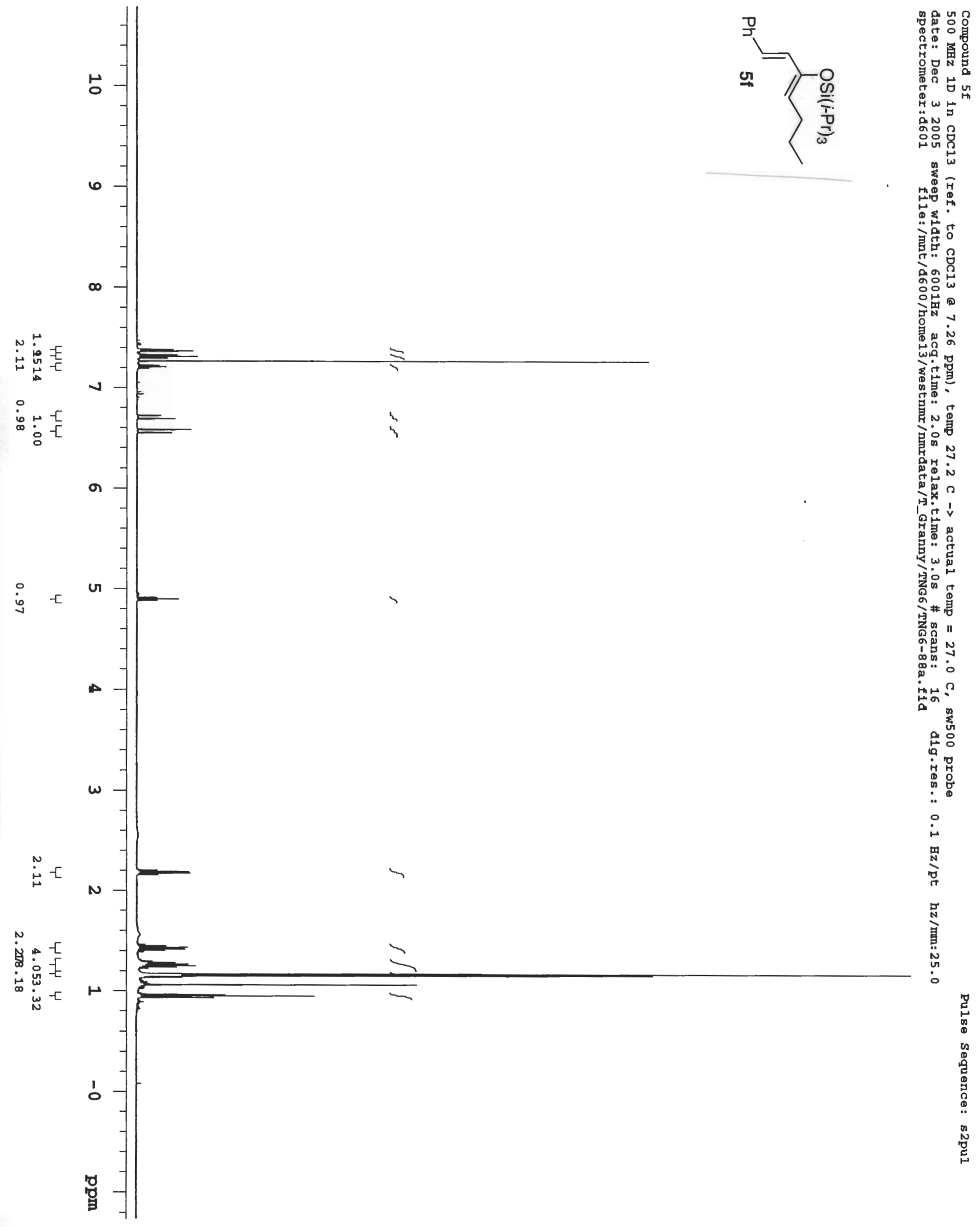




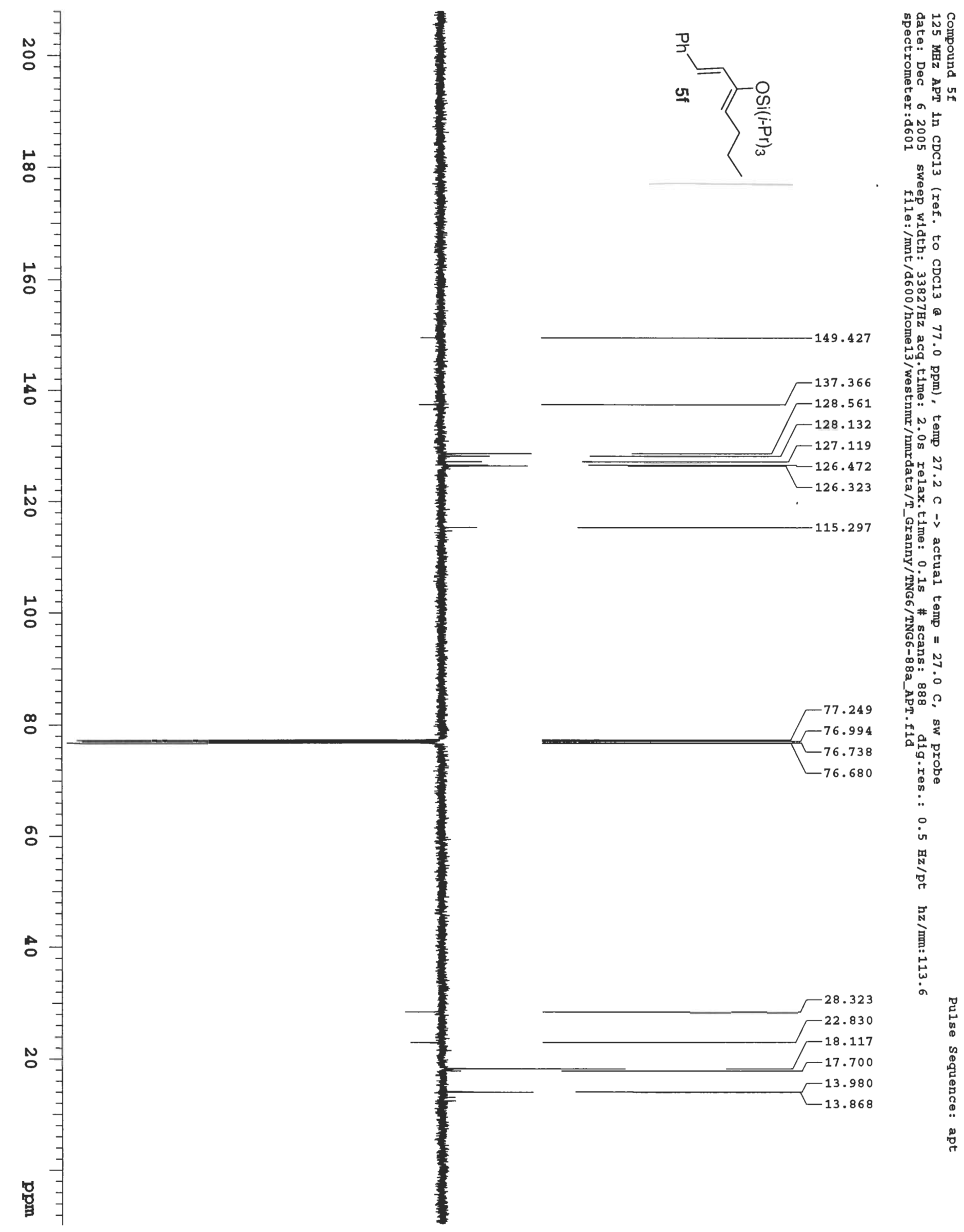




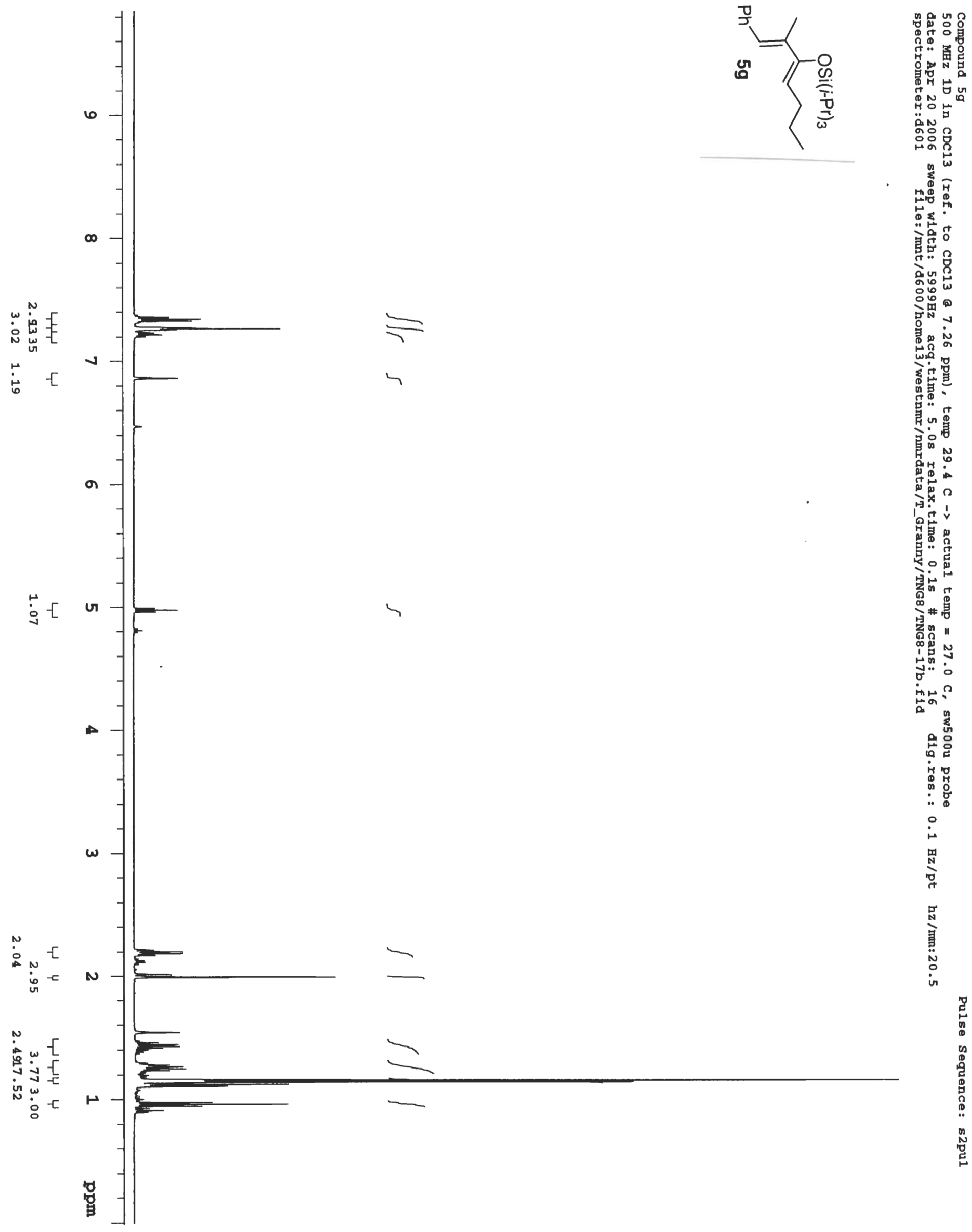




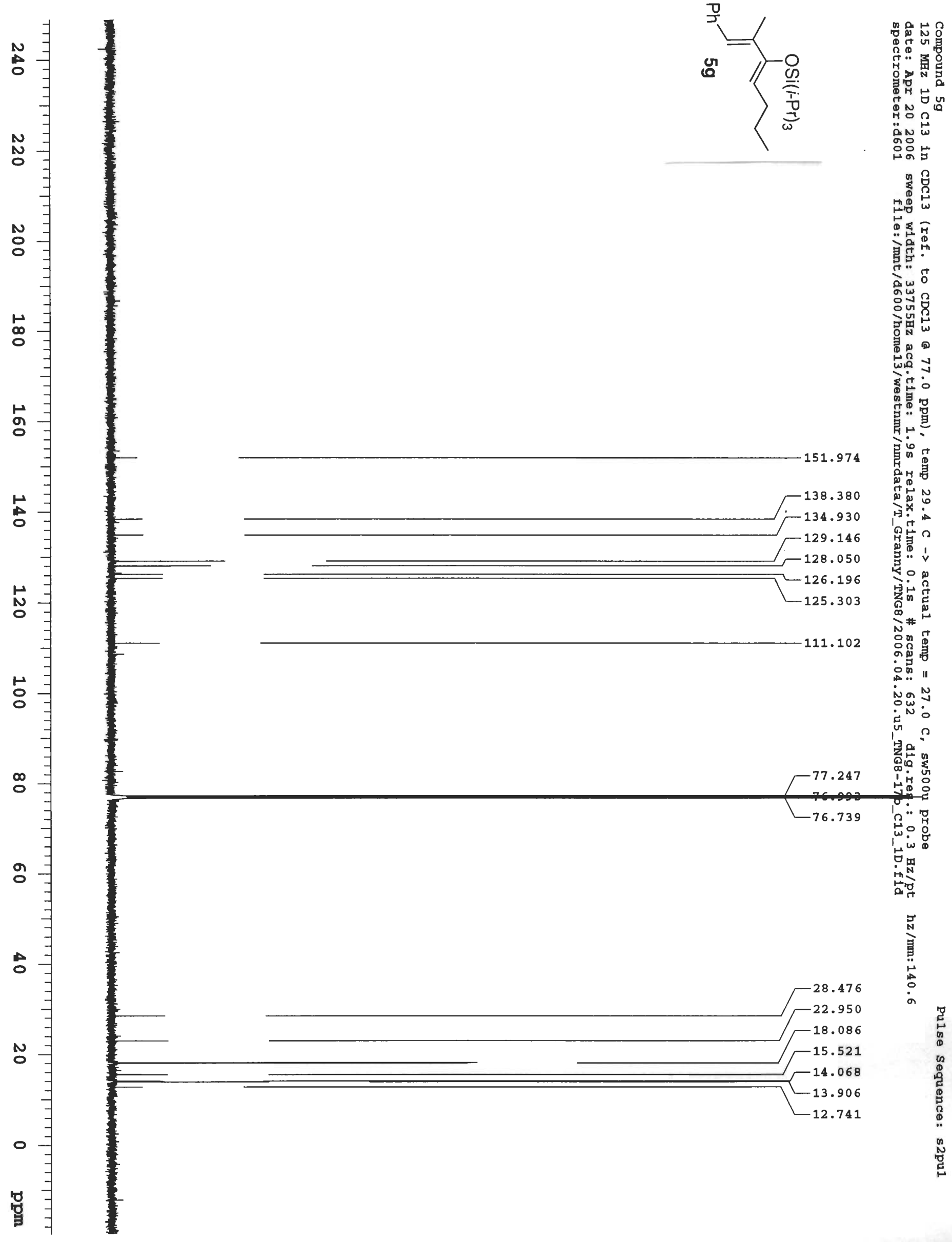




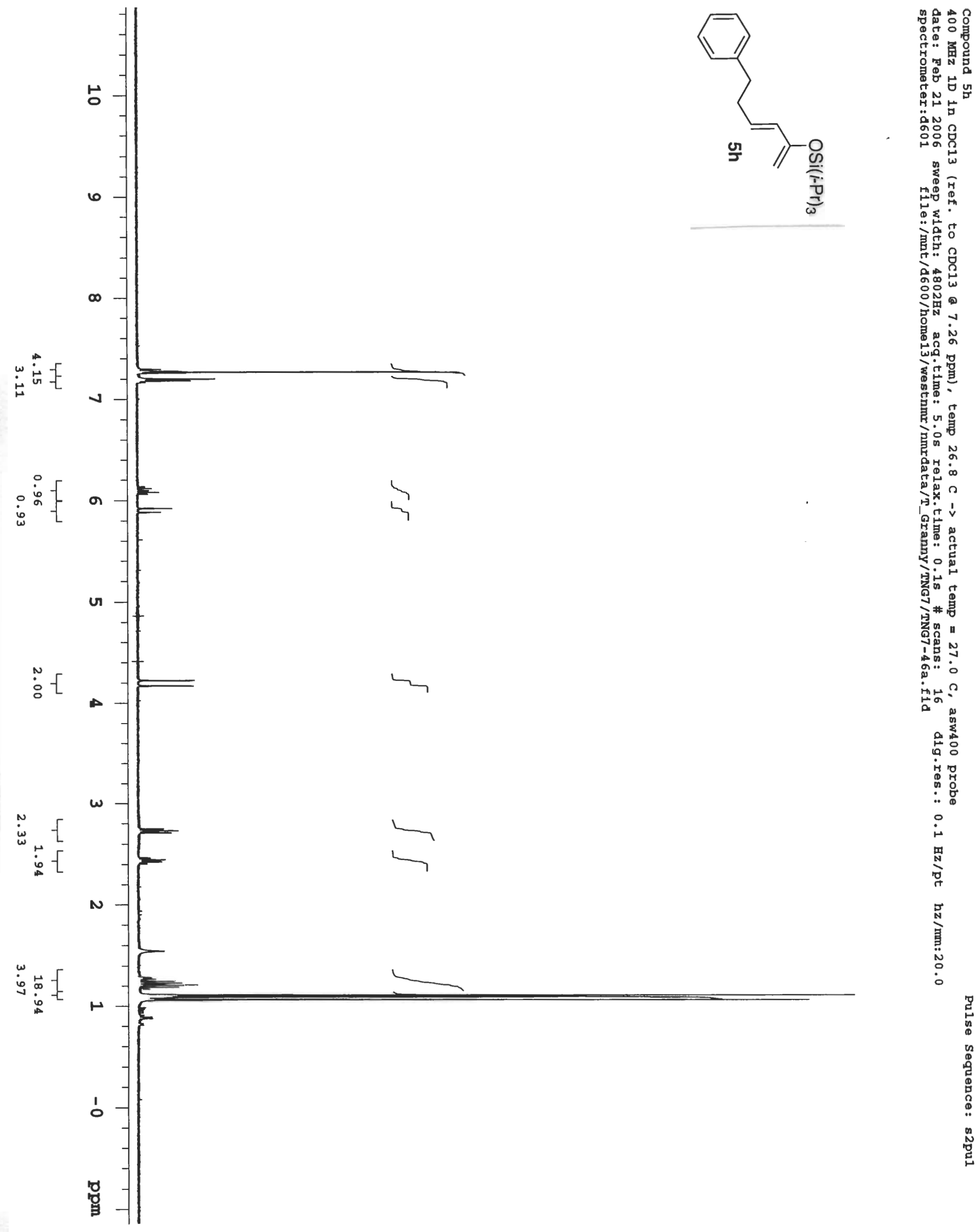




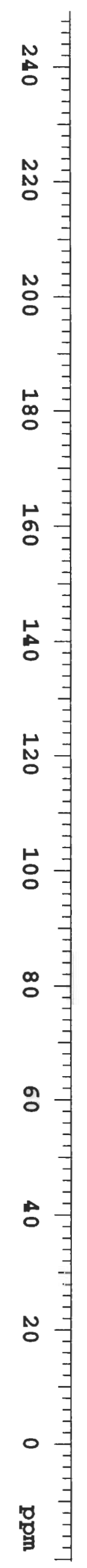

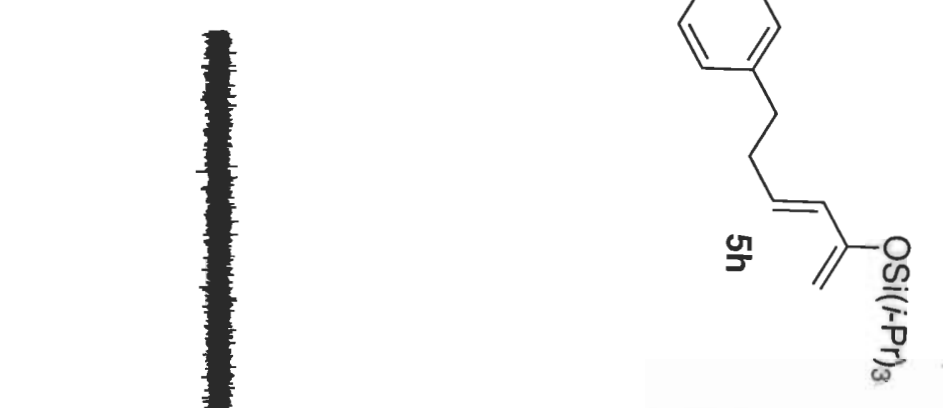

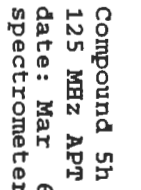

䓪菏

ㅇํㅇ을

虽

点点

เ्ะ

官宾敌

it

مू

w

贯

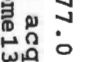

空它

品最

睤

155.257

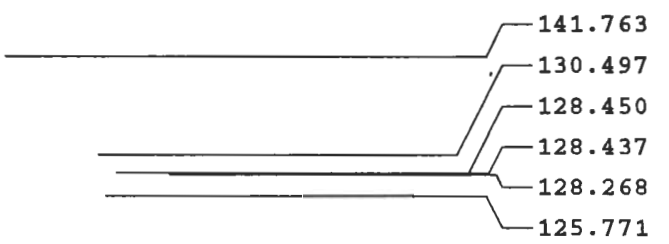

द

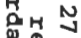

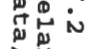

的。

원

灵茴。哭

实的总

党

舁界

คูก 11

会 N

.

ขू.

in

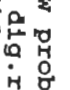

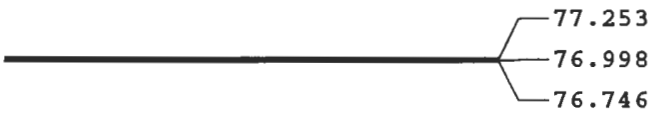

$\therefore$

in

思

竞 


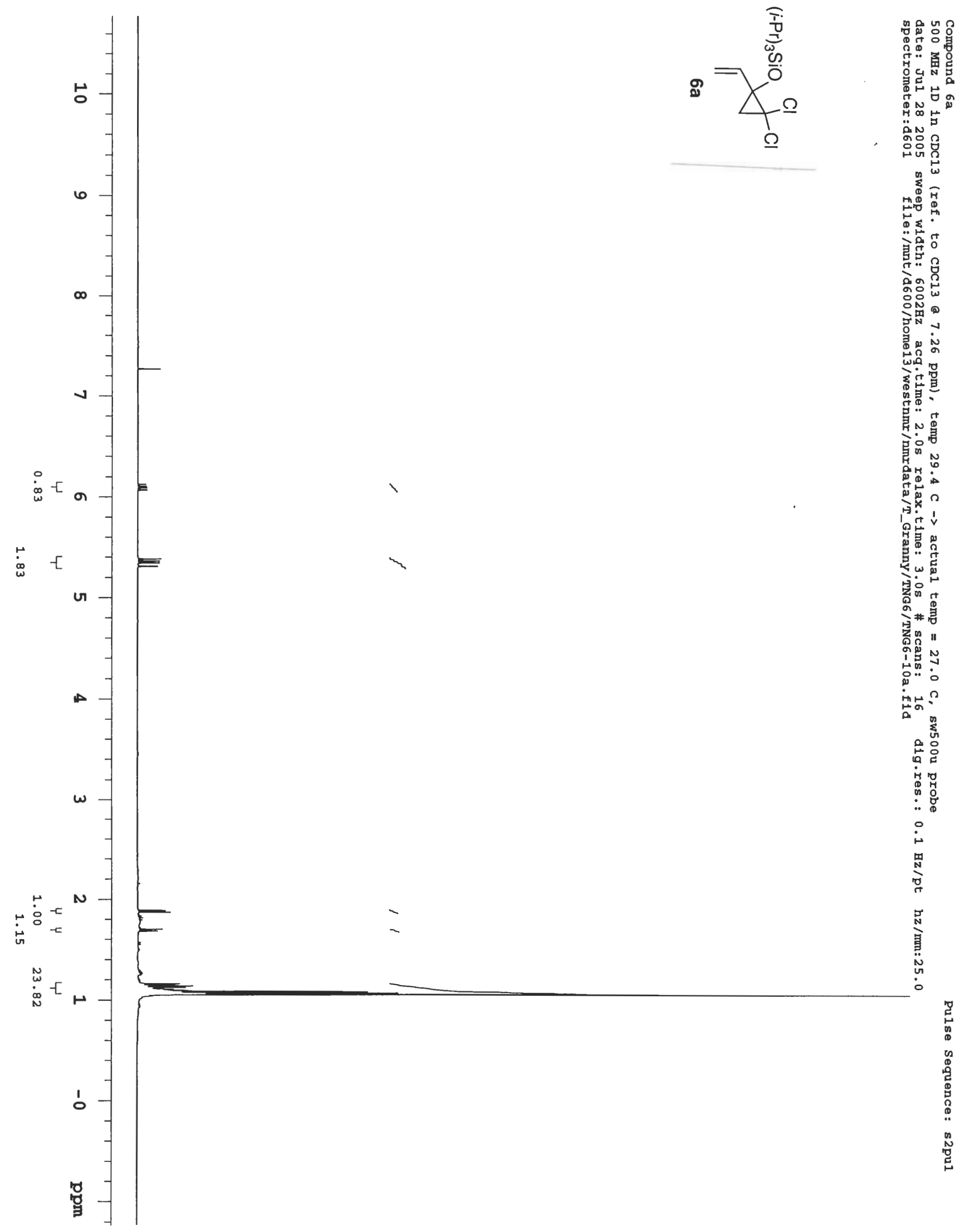




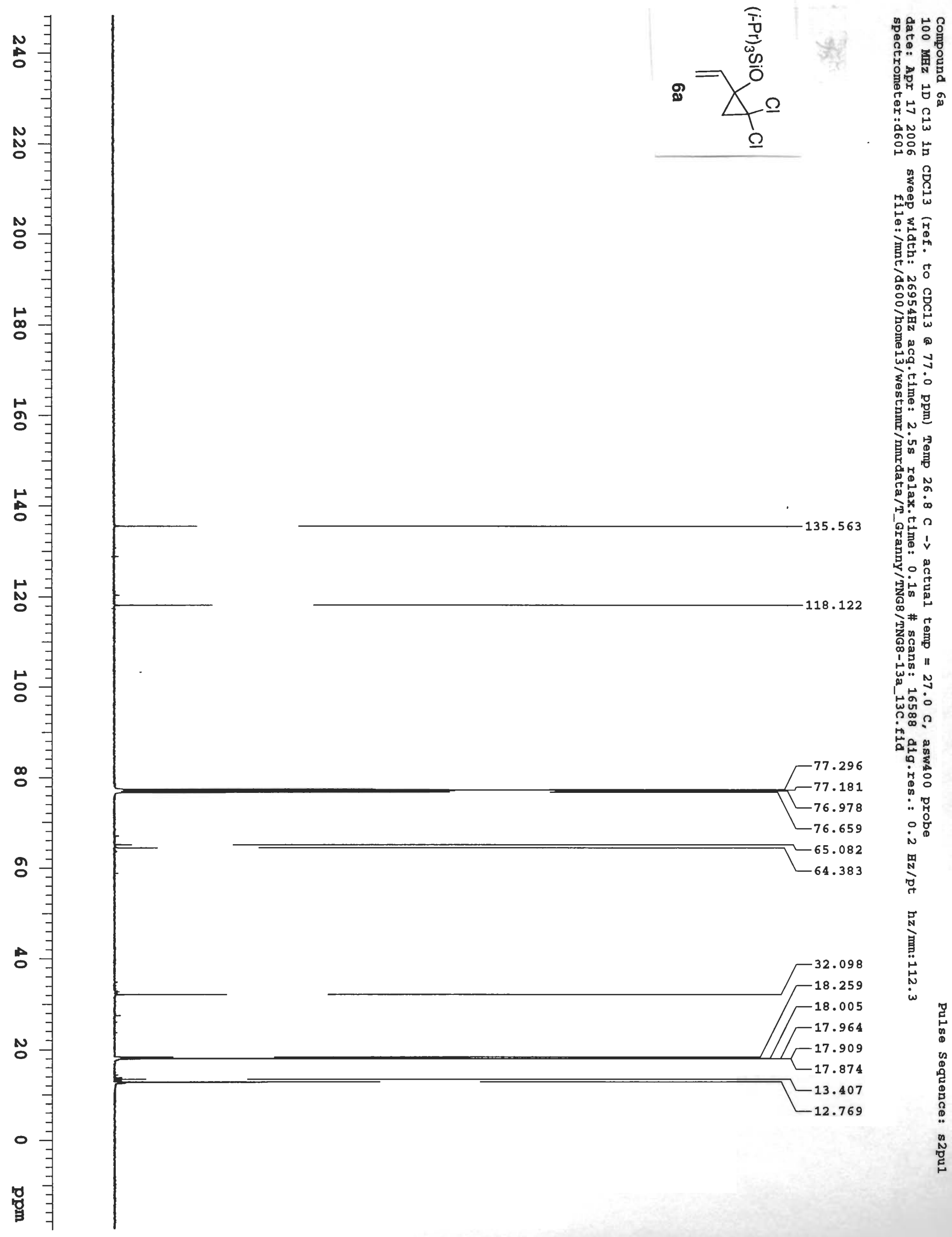




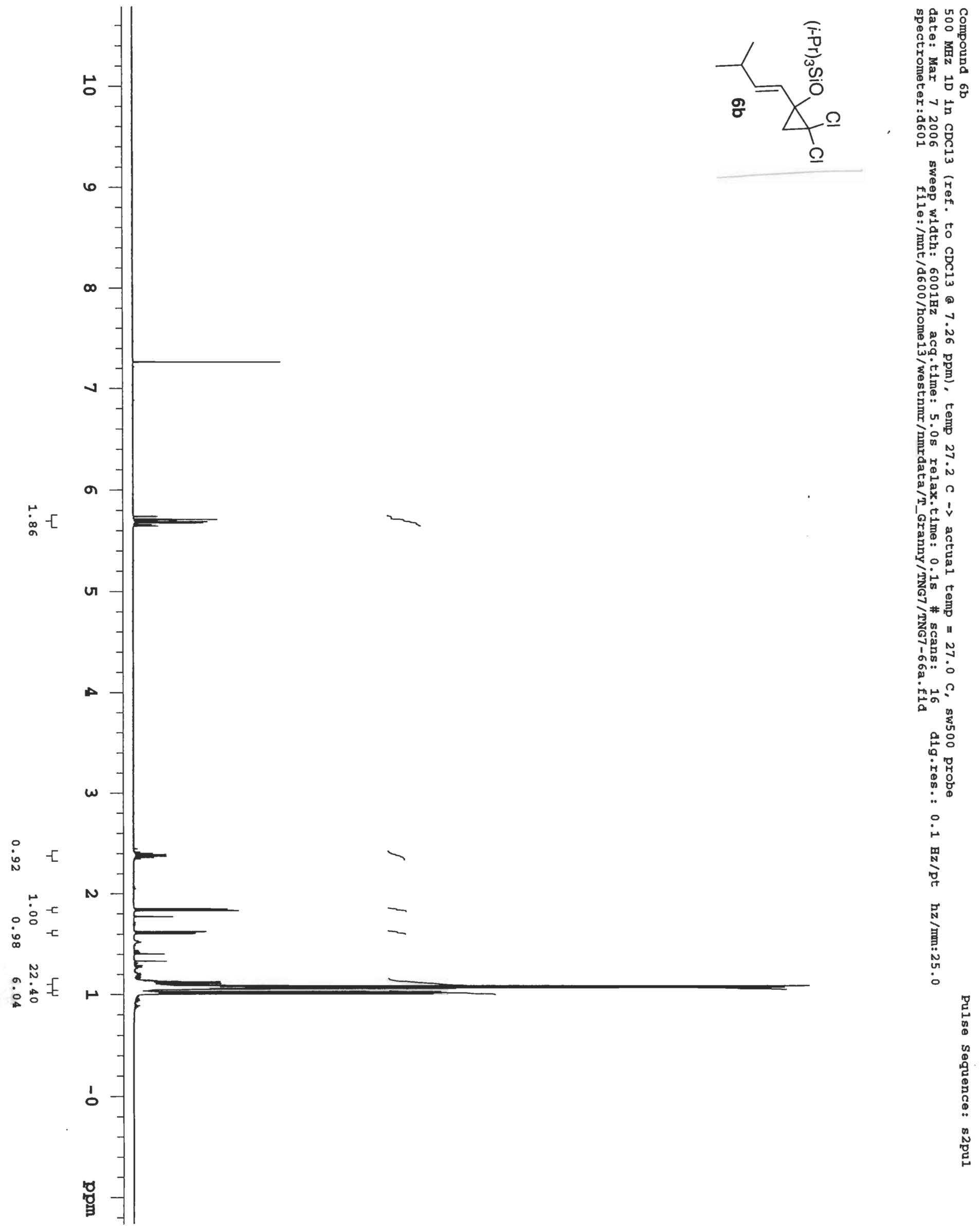




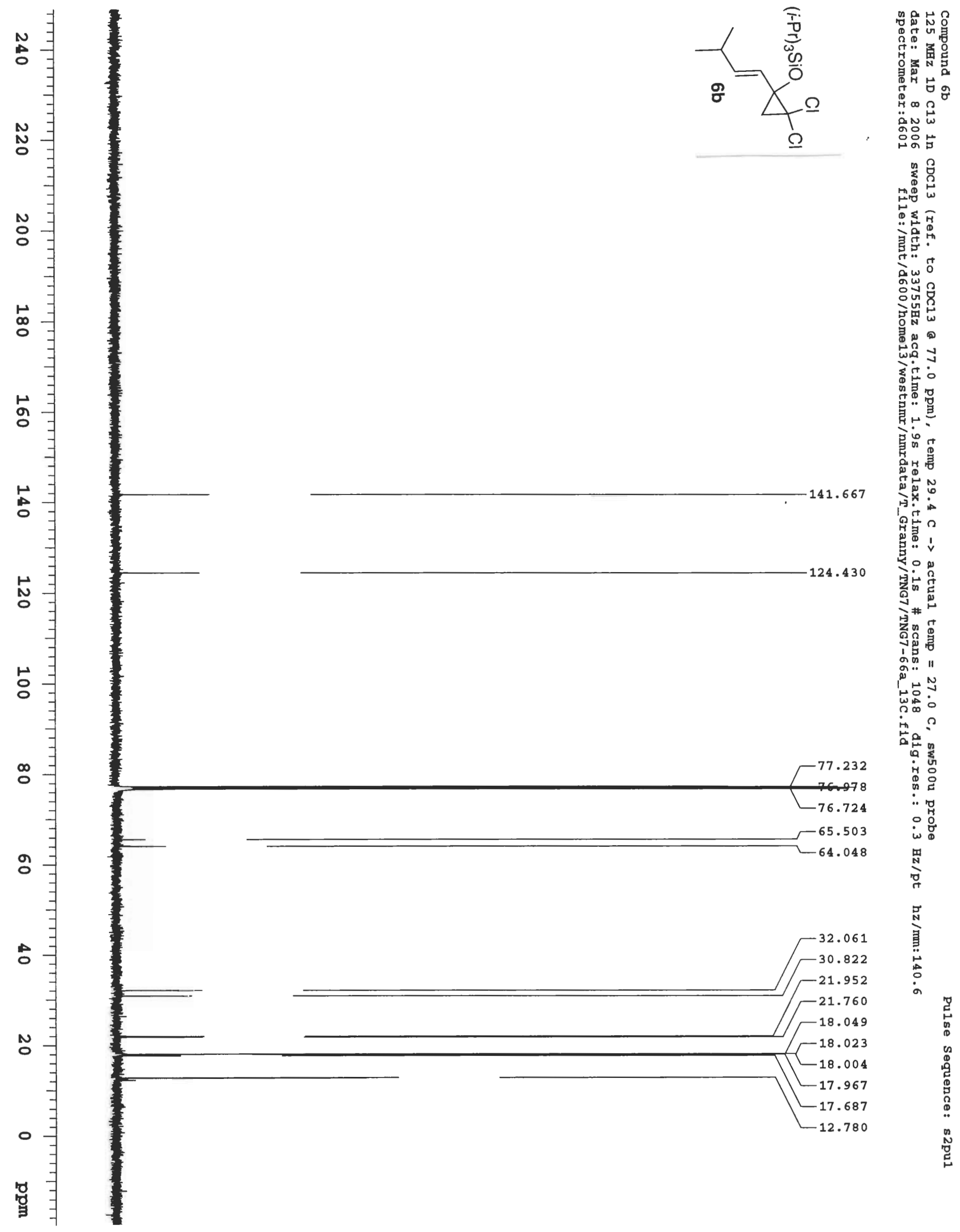




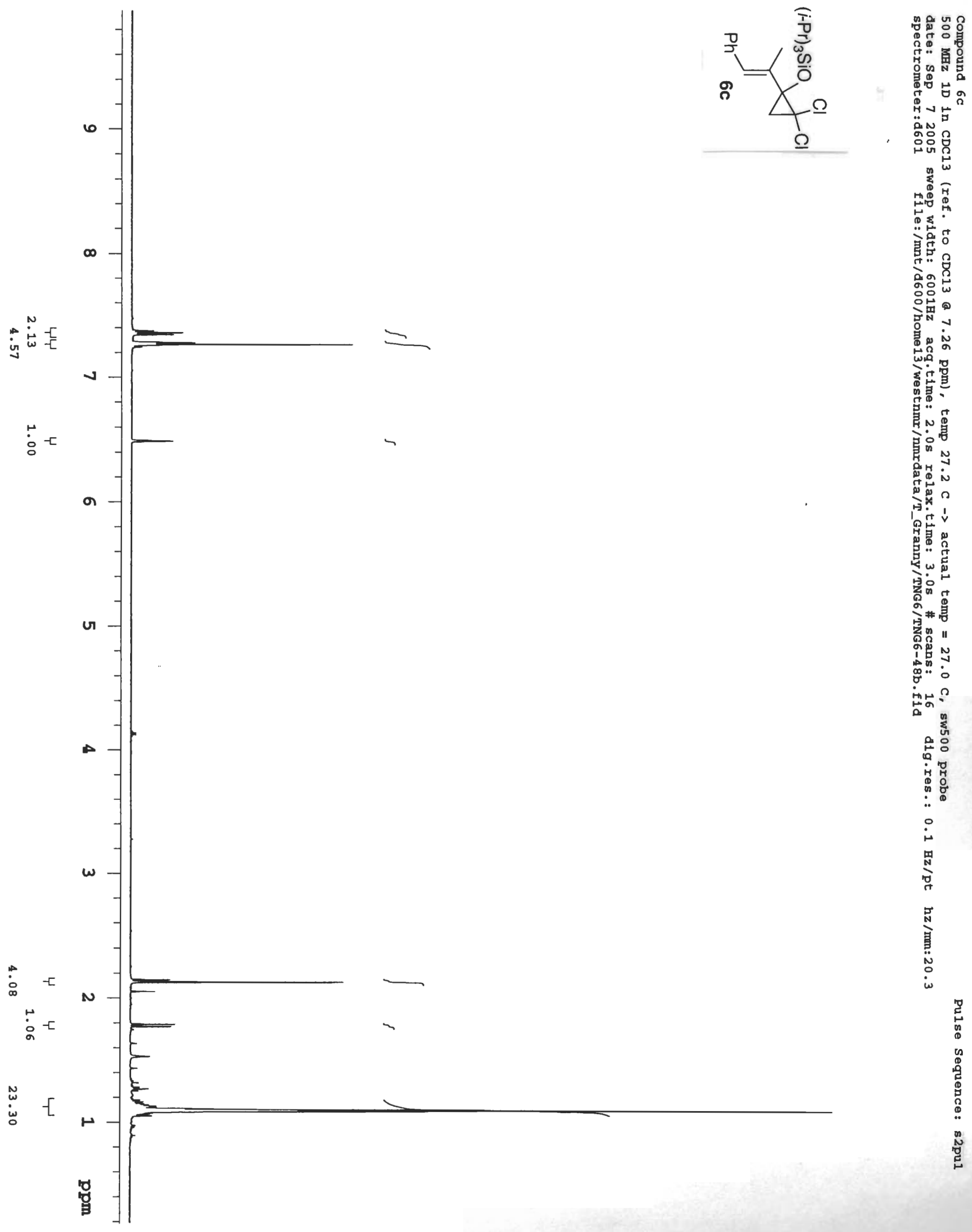




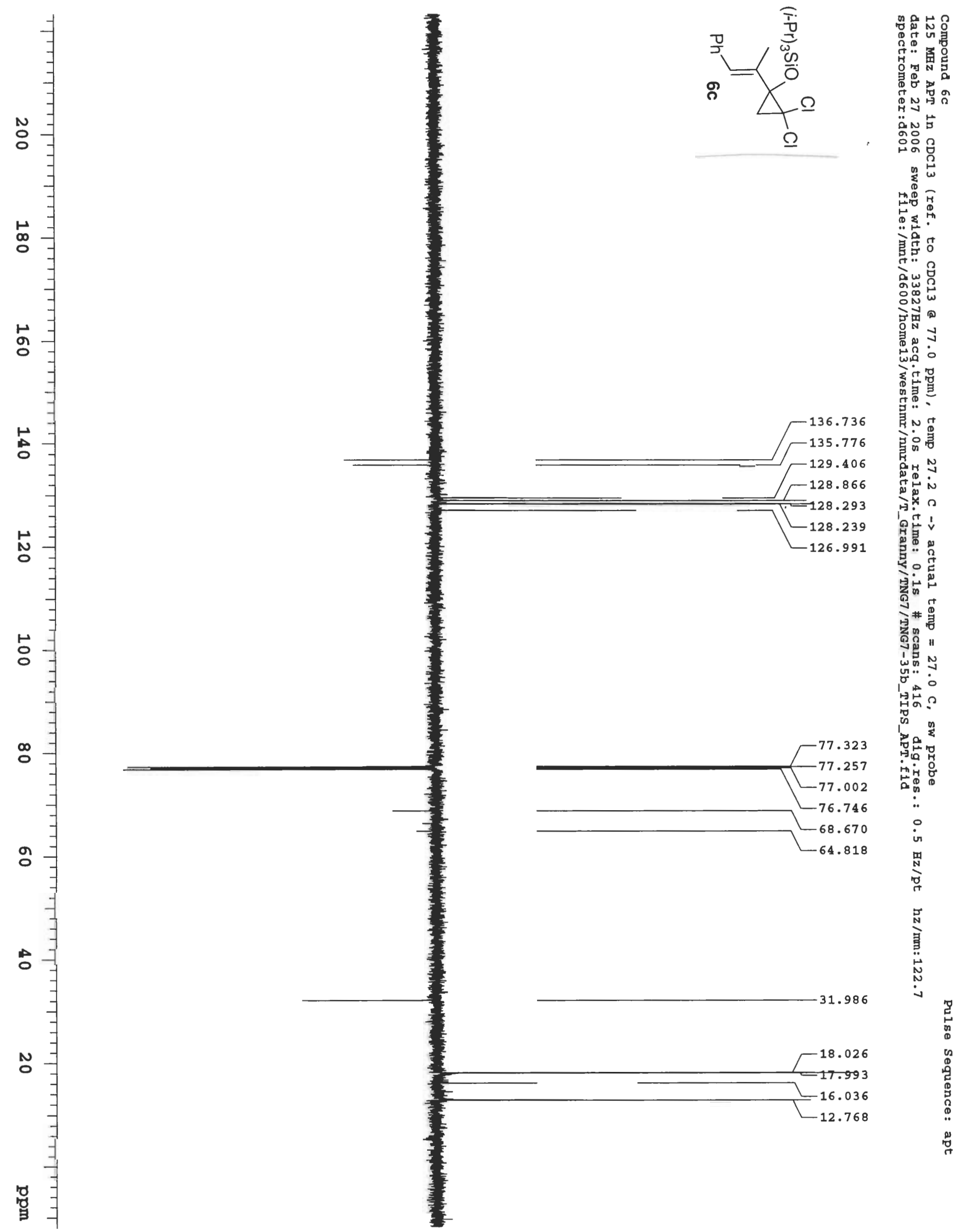




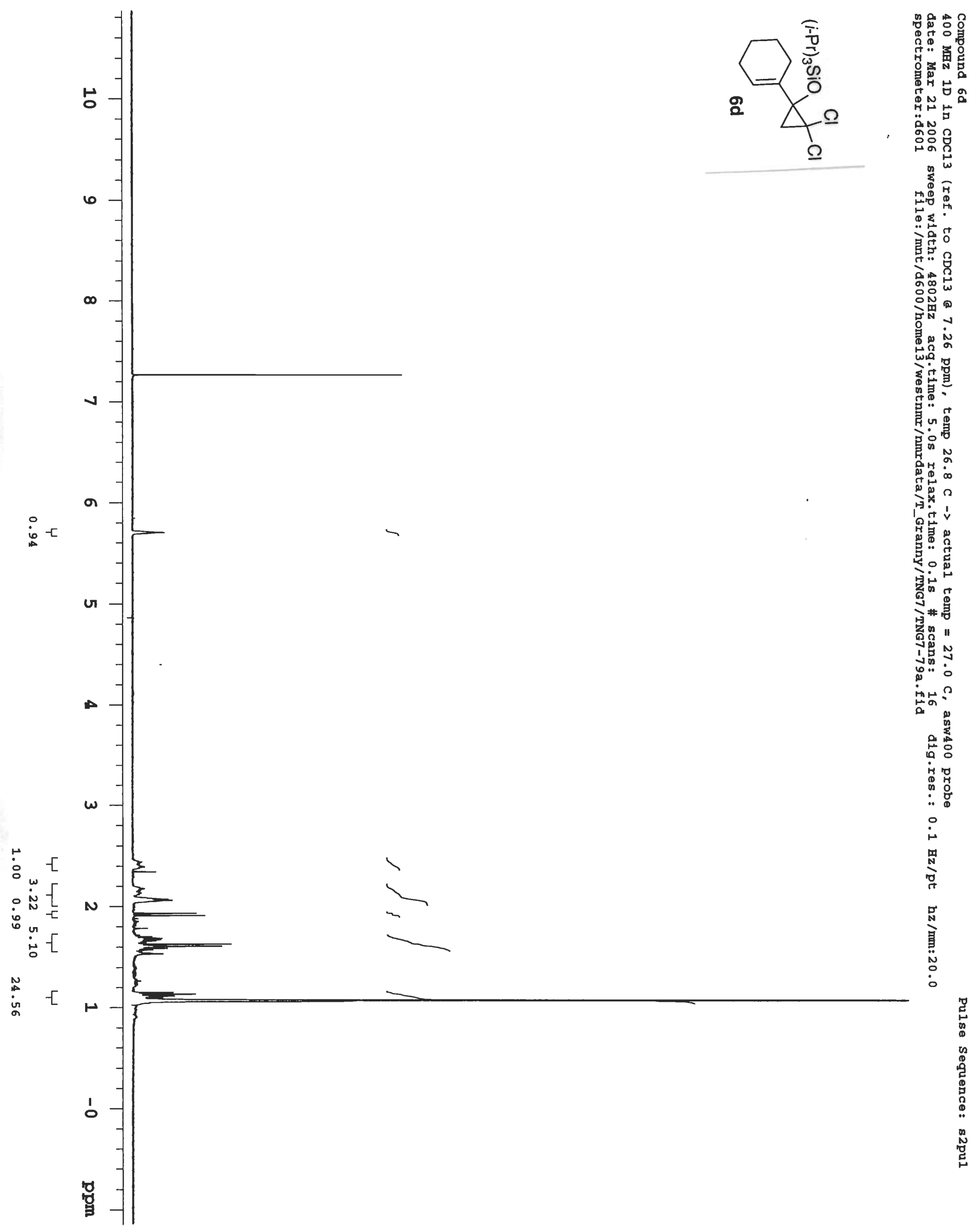

S-34 


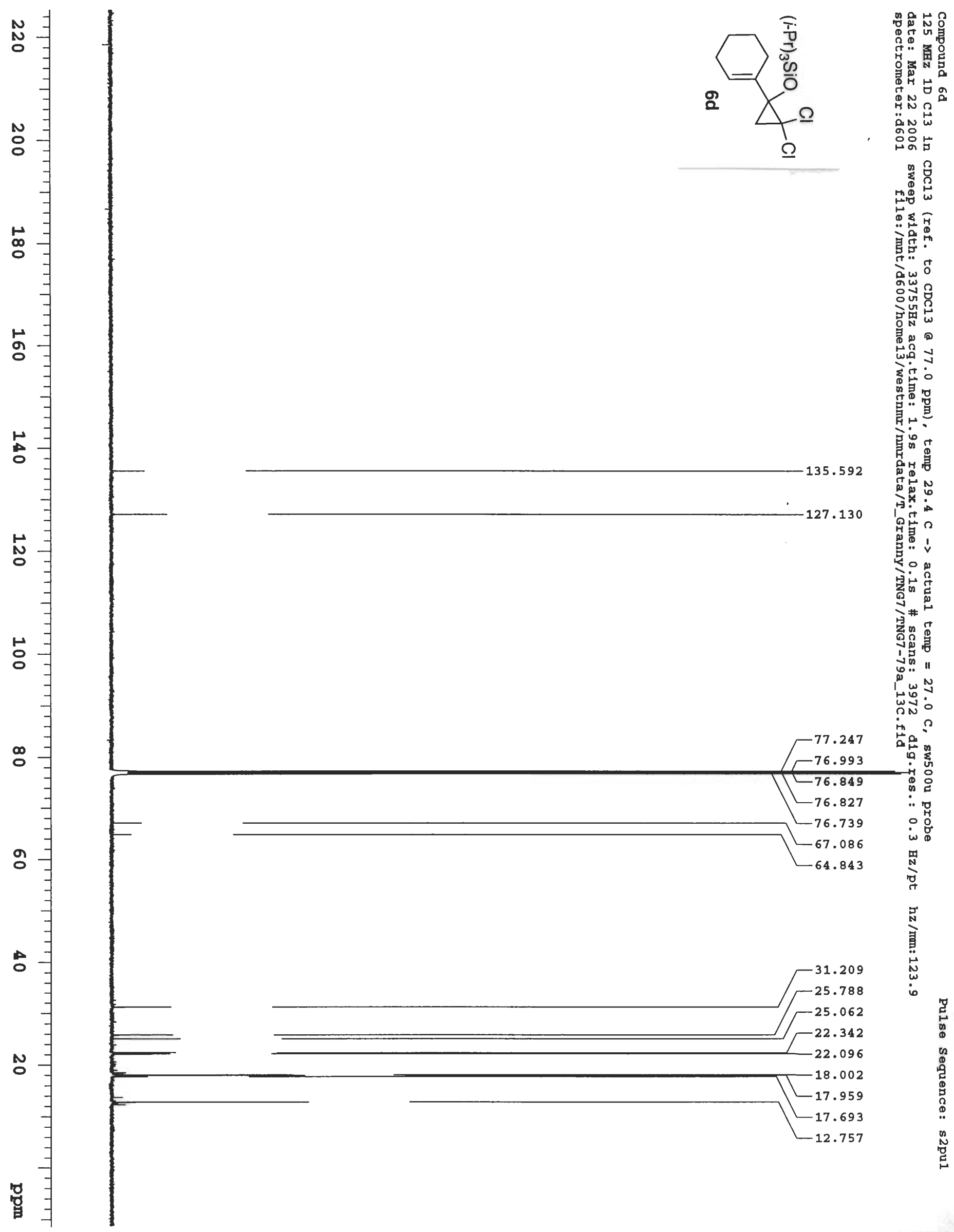




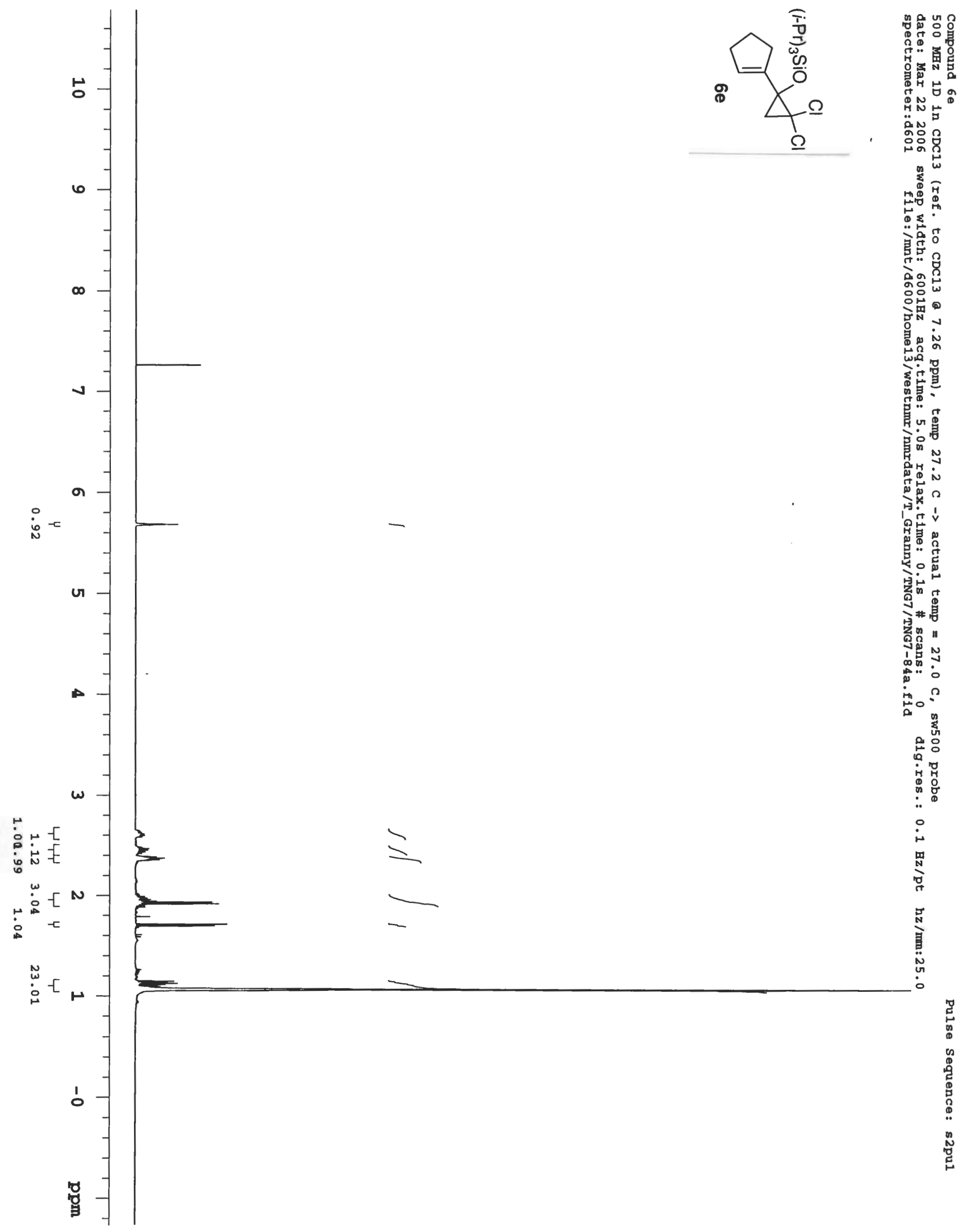




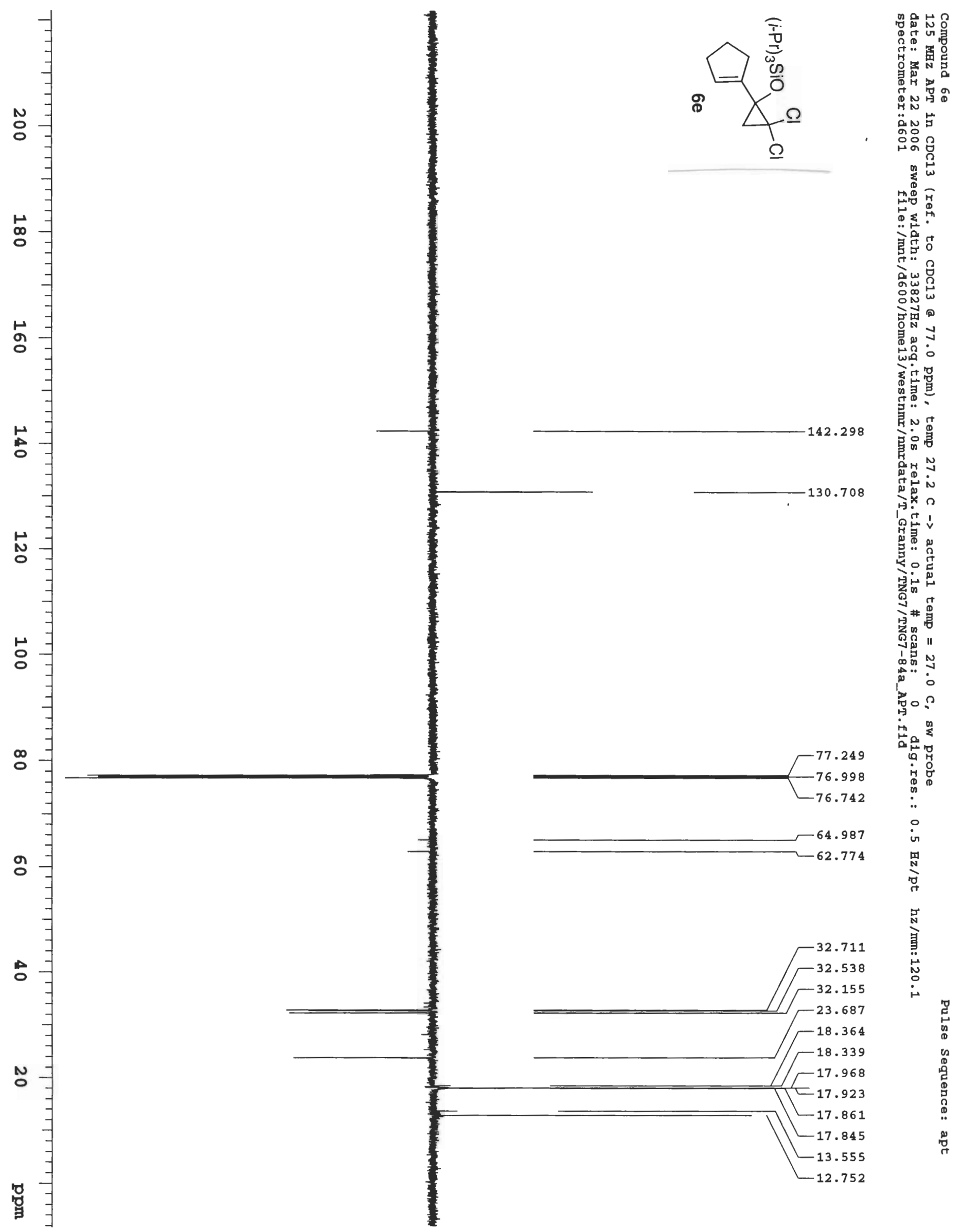



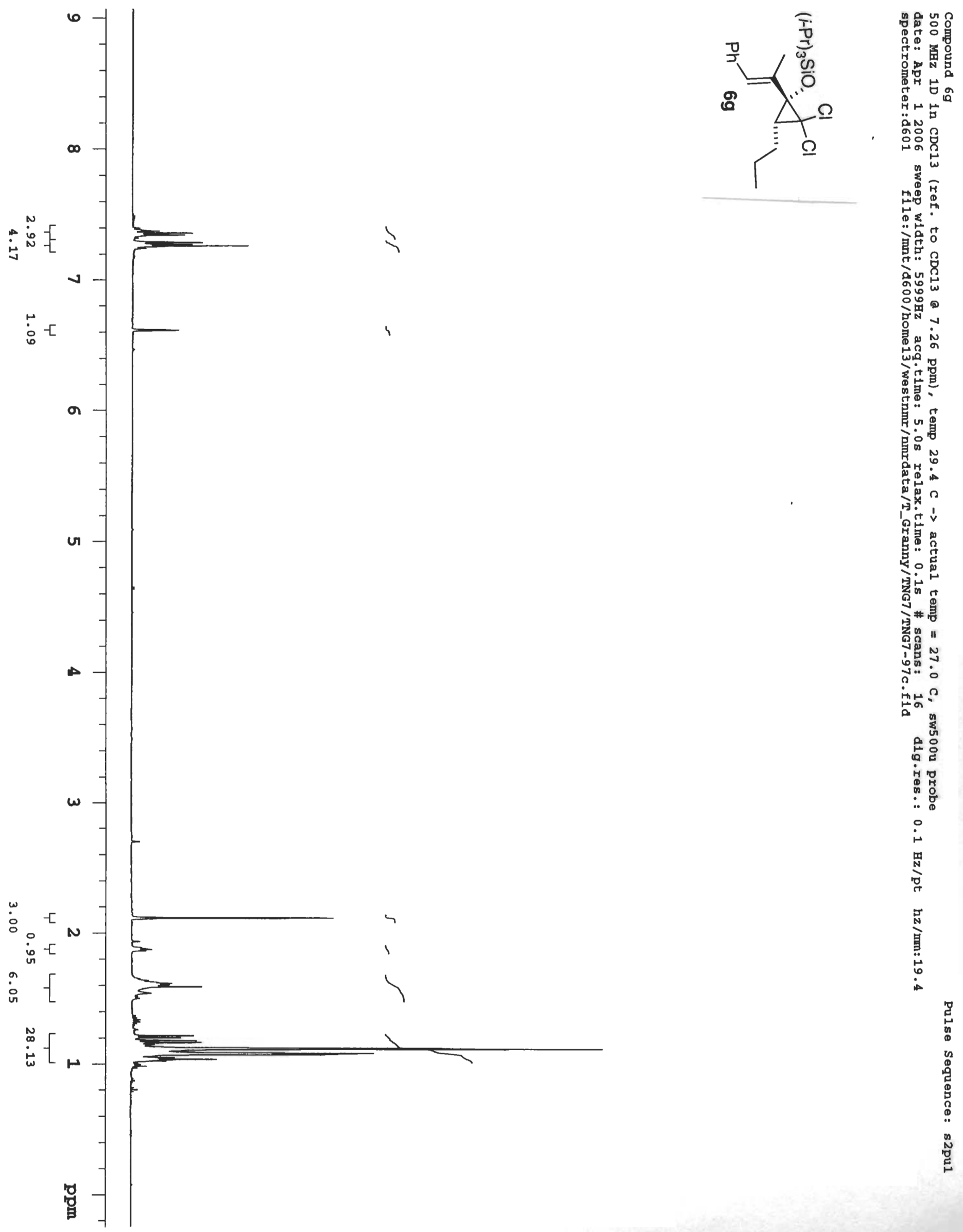


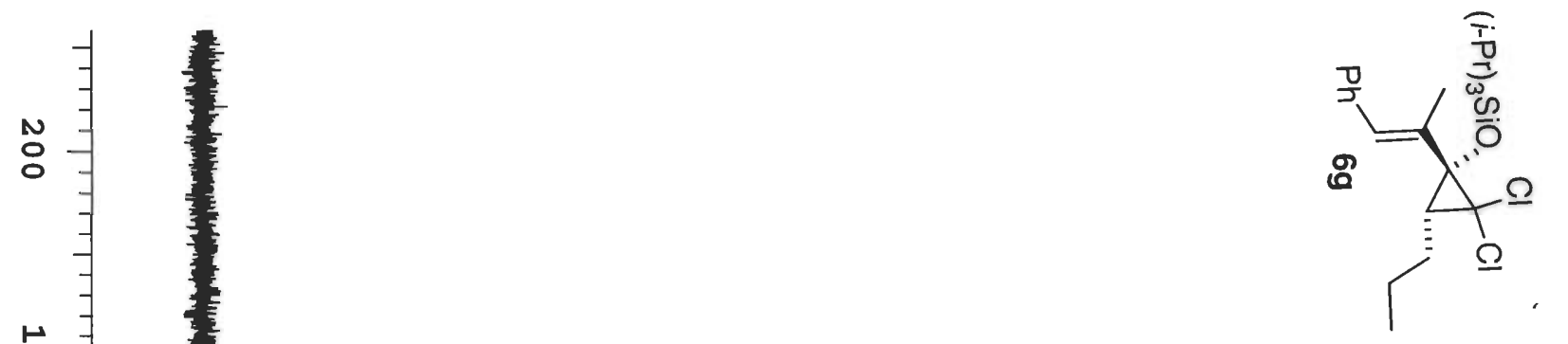

$n$
0
0
0
0
0
0
0
0
0
0
0
0
0
0
0
0

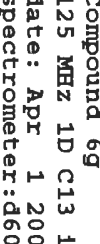

응음

ะี

思离

옹

官要.

茣

홍

$\stackrel{⿱ ⺊ 口}{\circ}$

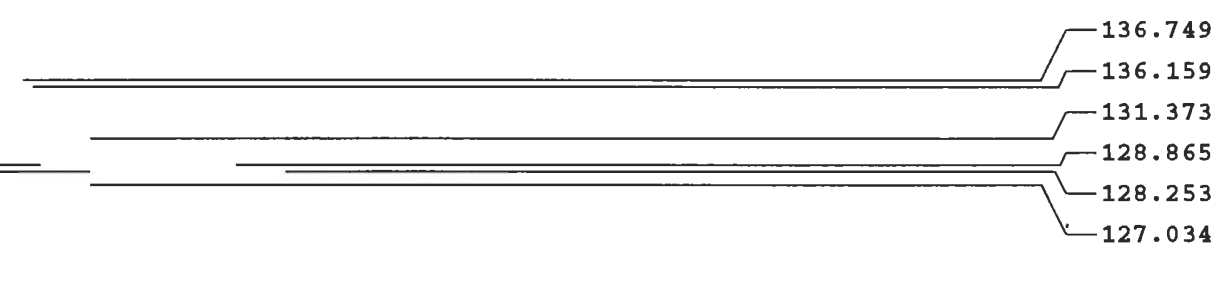

숭

0
0
0
0
0
0
0
0 .

$\infty$

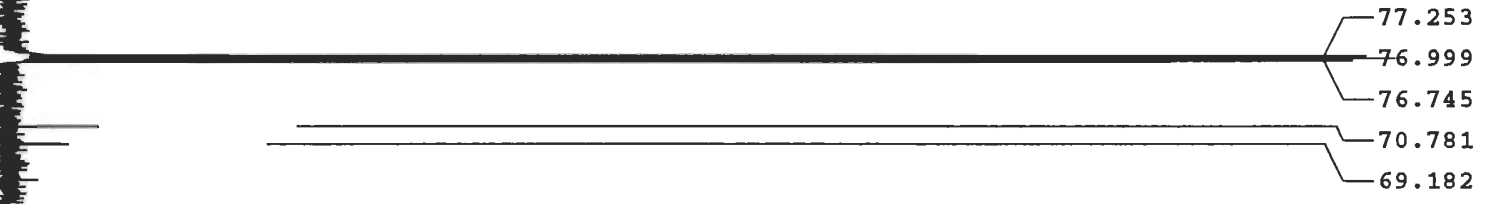

a

○

.

-

N

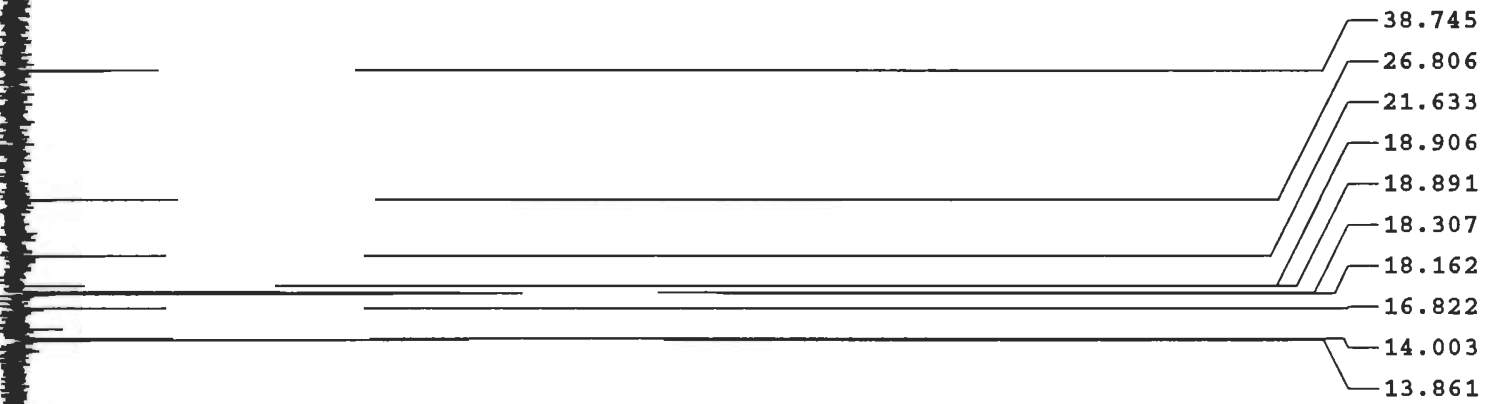

웡ำ

罢点

究

ผด ป

安它

总兽

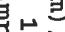

灵占

总

苋

is is

葛

है

○

密的

\#

实的

10

ㅇ..

مै. N

岕:

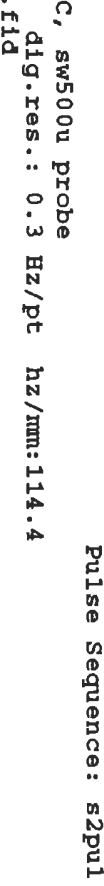

总

0

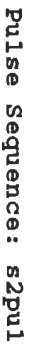




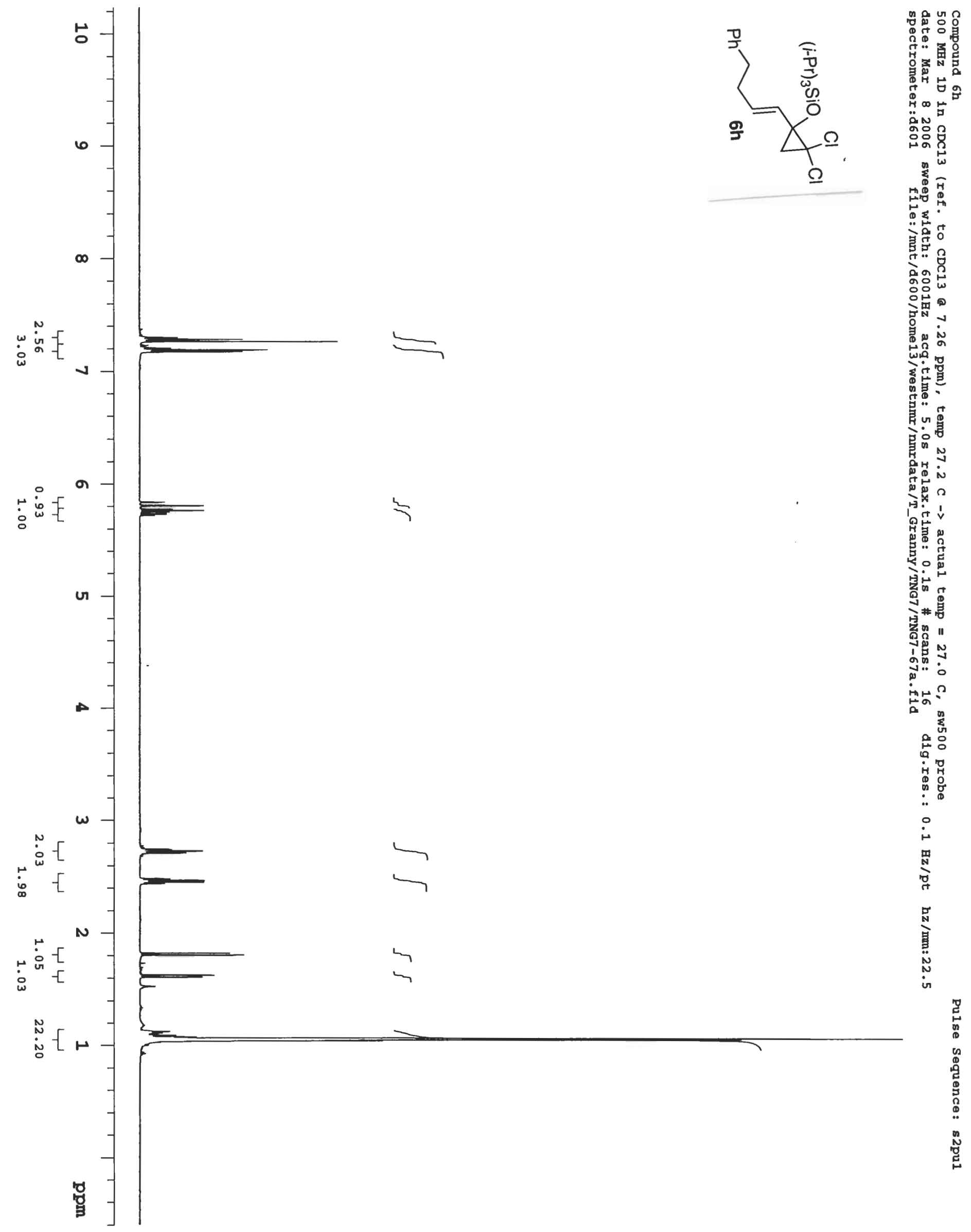




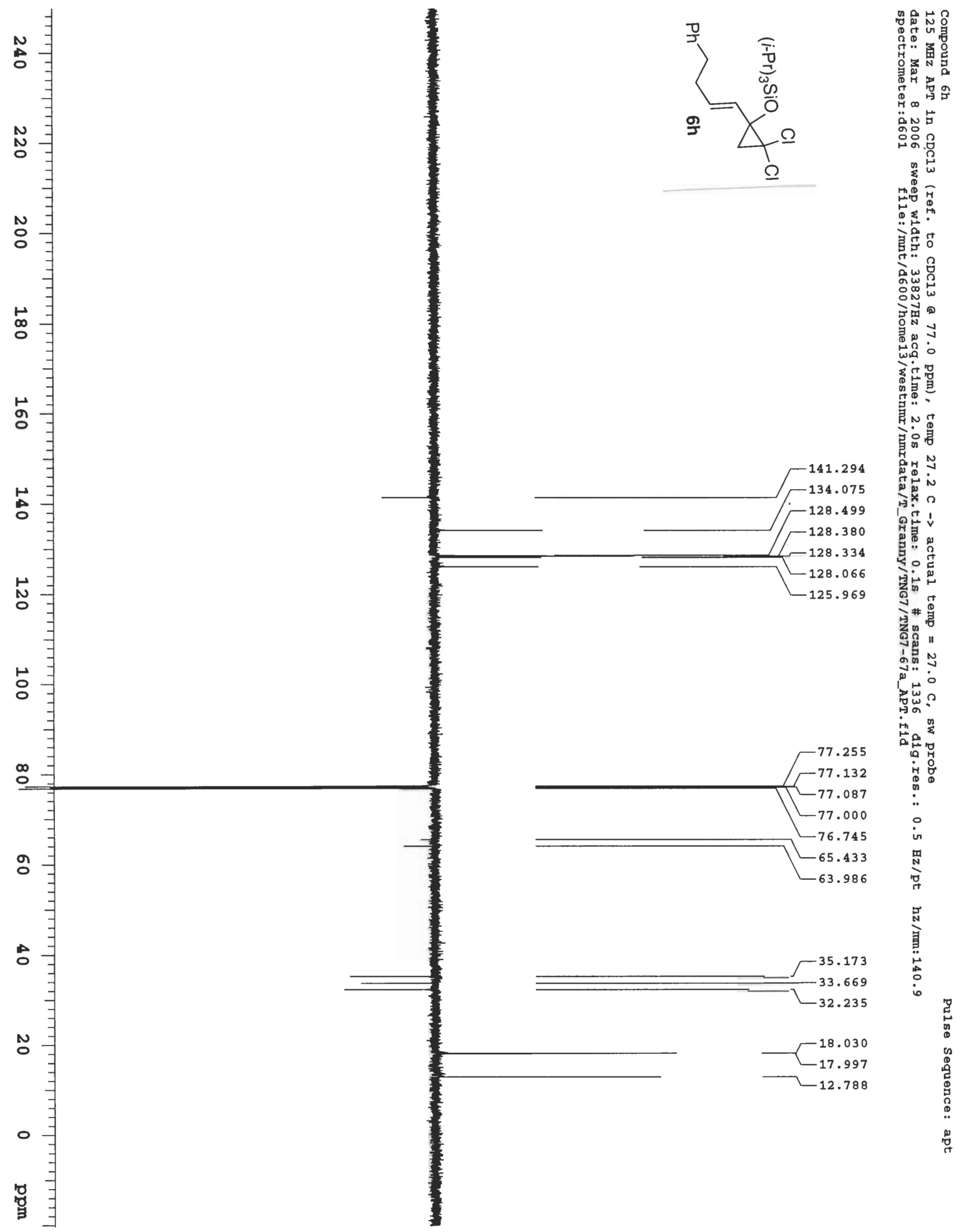



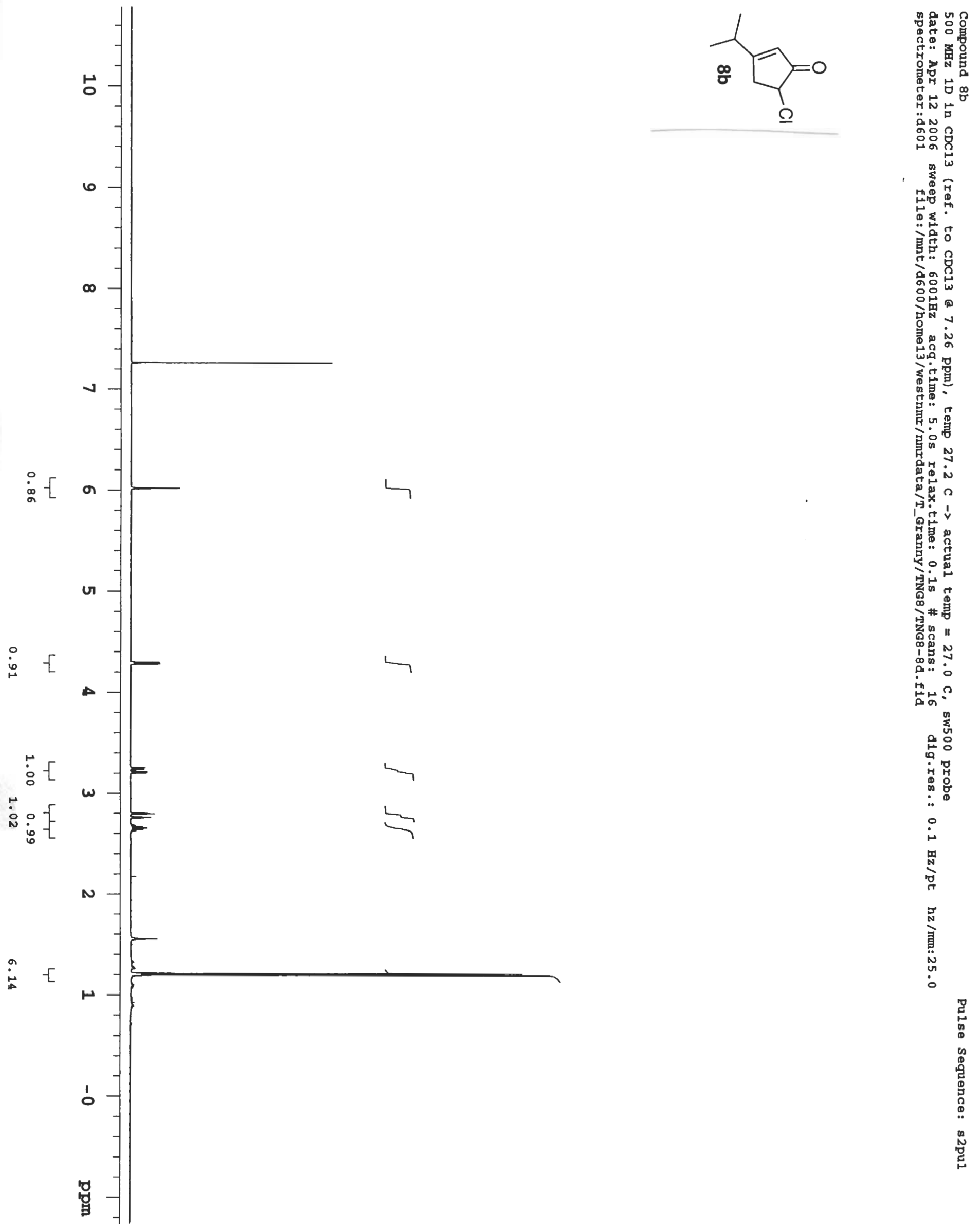

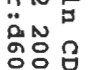

ด

o

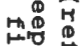

कह

实哭

‥

ㅇํㅇ을

踾

‥

足。

的它

on

䡒 u

定品

군

年

点

定。

安的

\#总

ด้ "

i

कo

蛅?

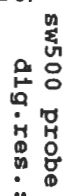

.

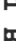

윰

章 


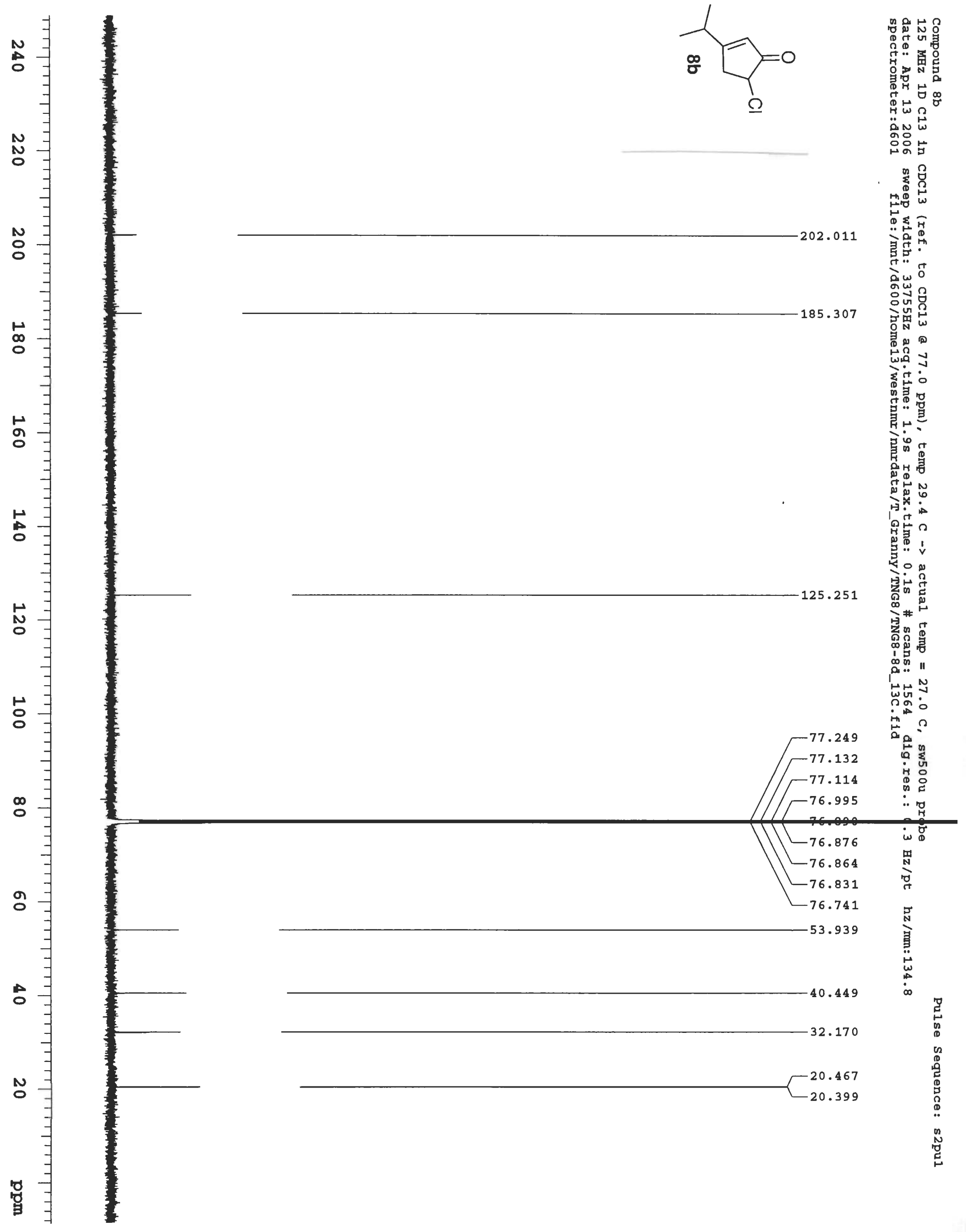




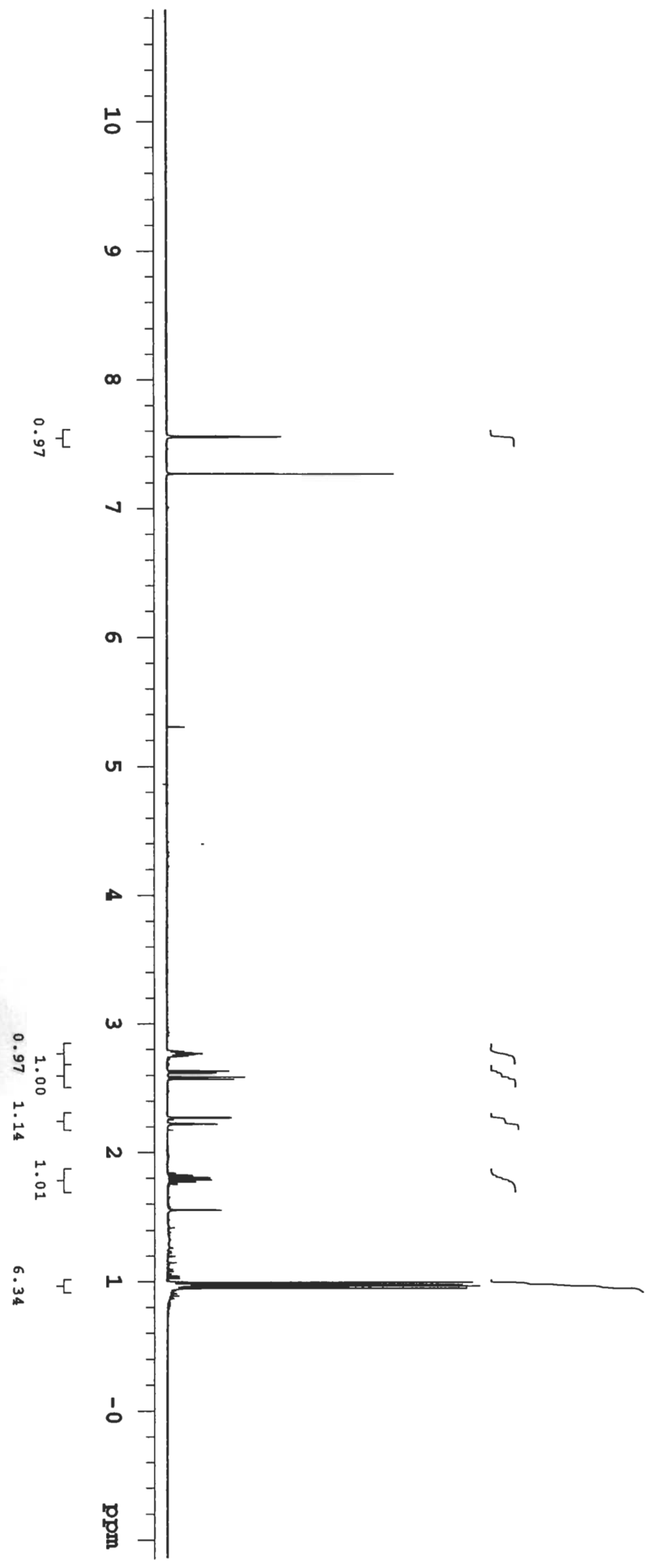

엥요

年要 兽要

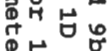

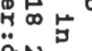
옹용 คด 离点 要 क 氙

品 an مิ 욤 पू 最哭会 w

它包 政 吸 安星 察 W 造 A क 星 定。 感 离 药 政 A. 蛅? 


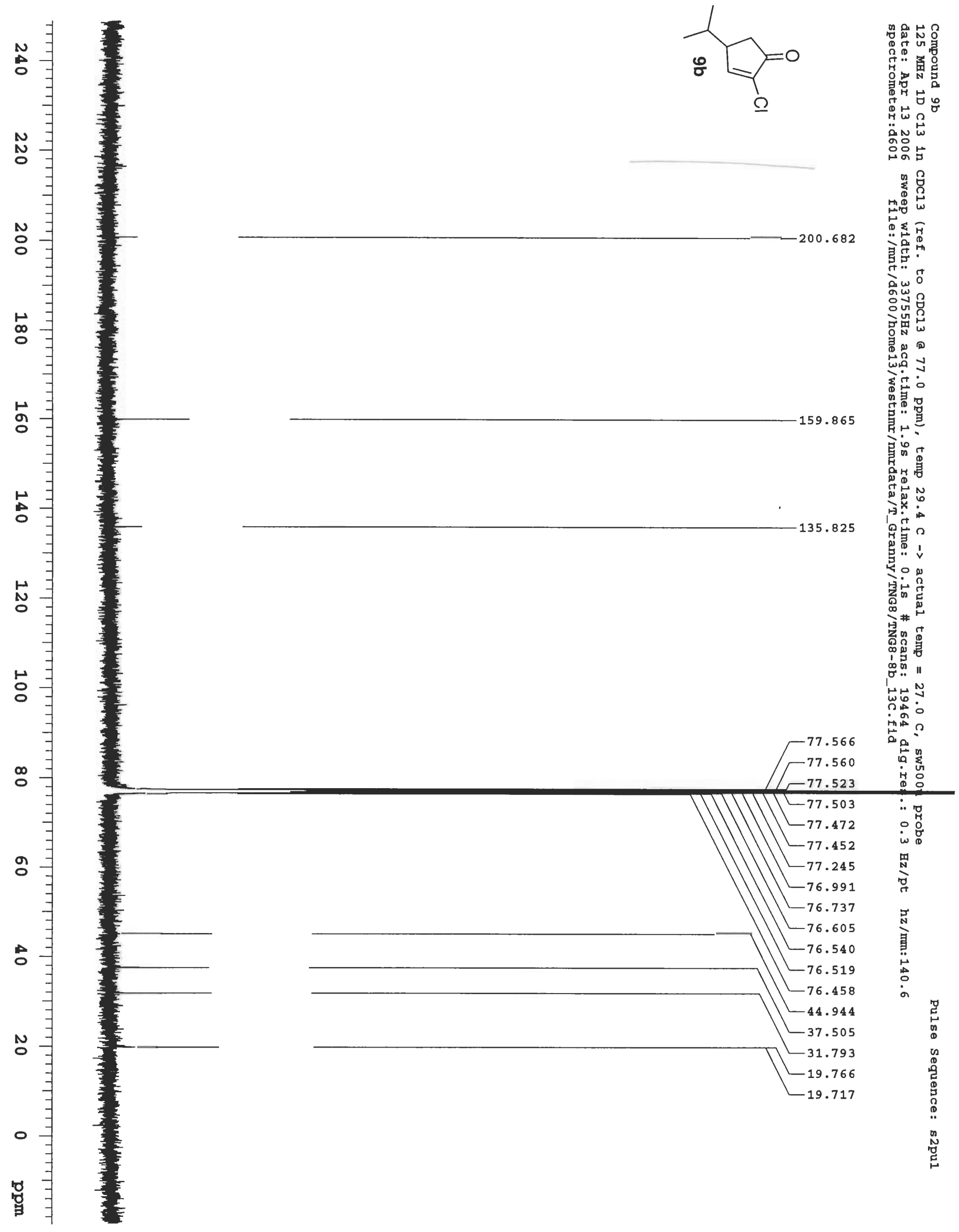



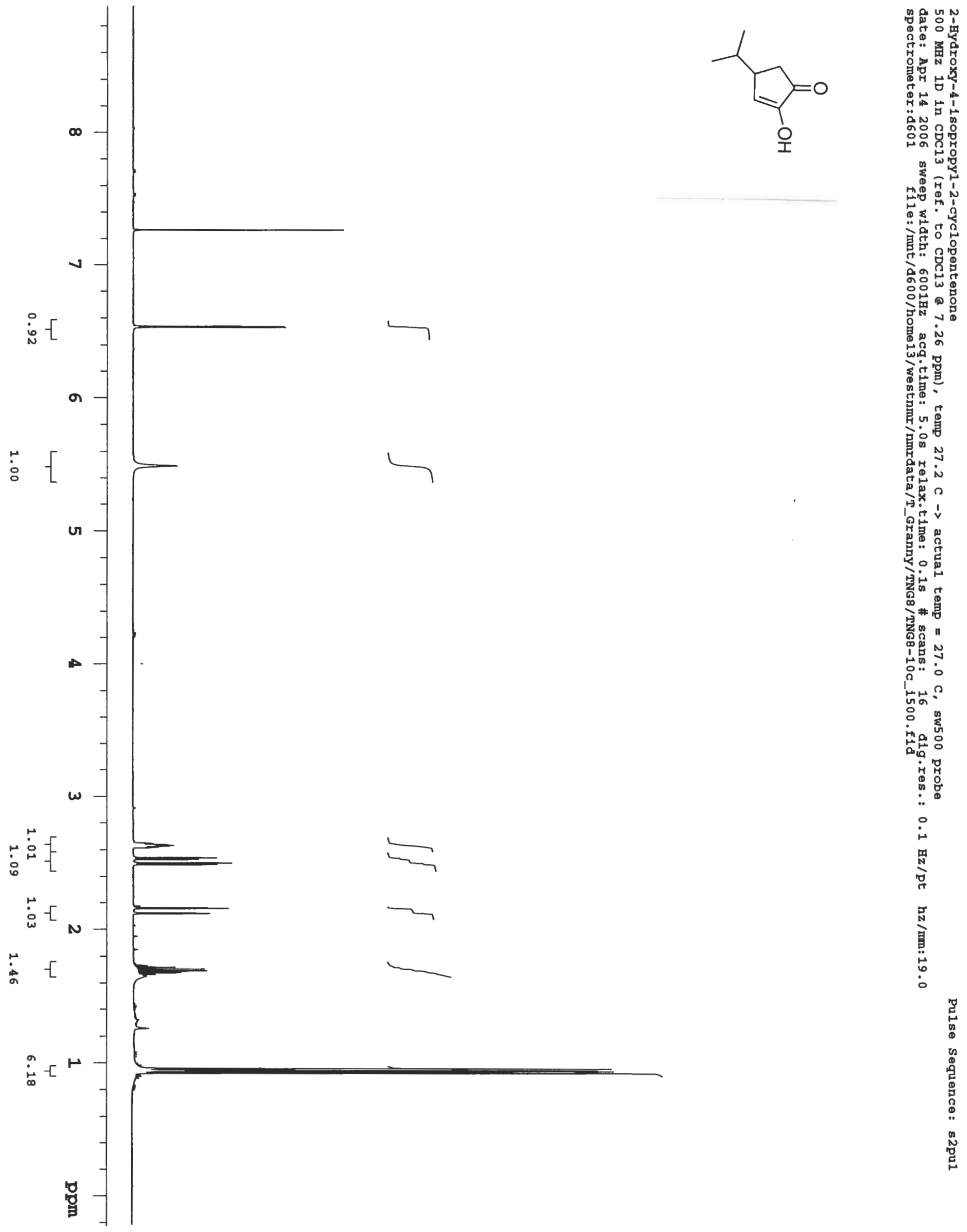


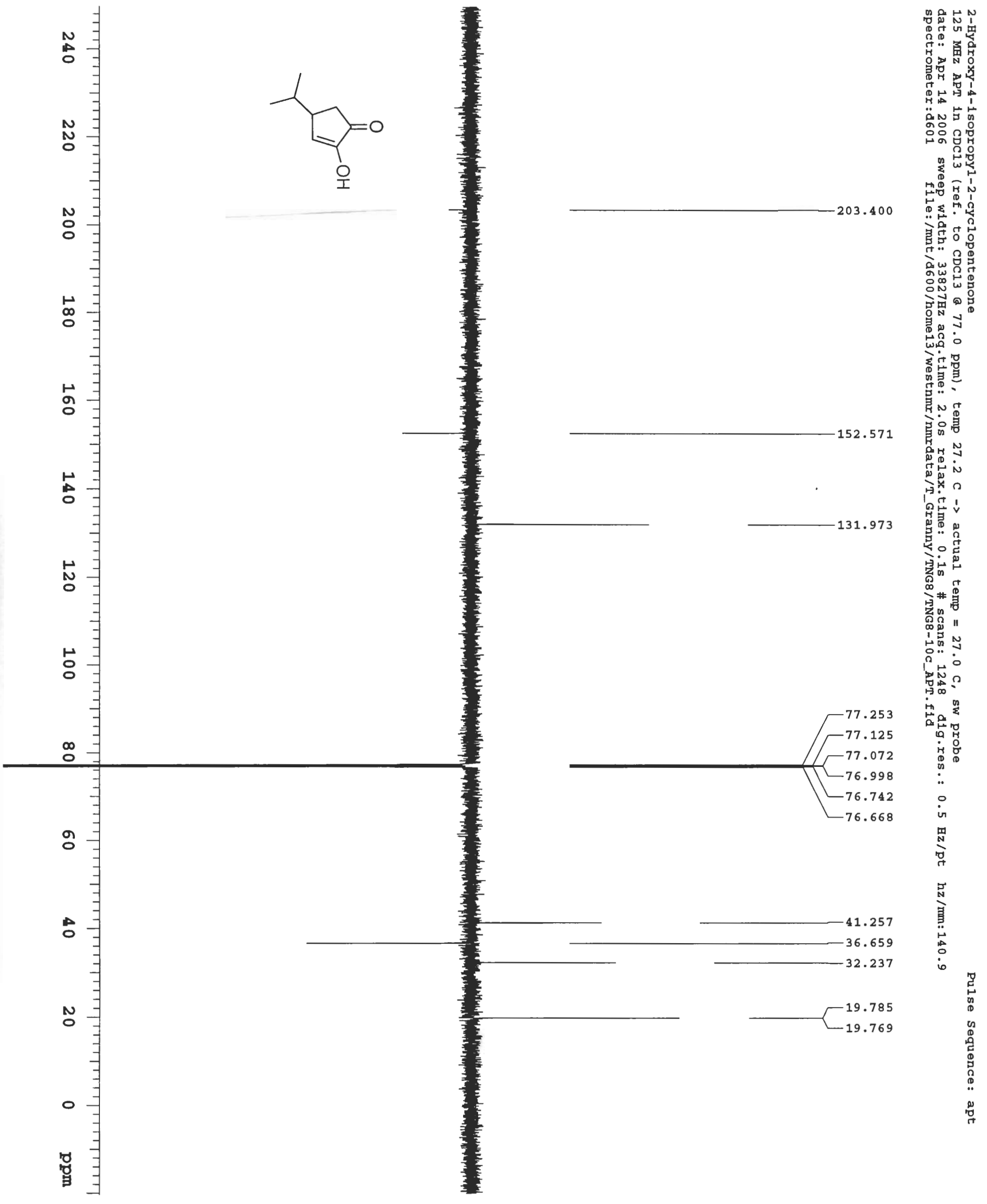



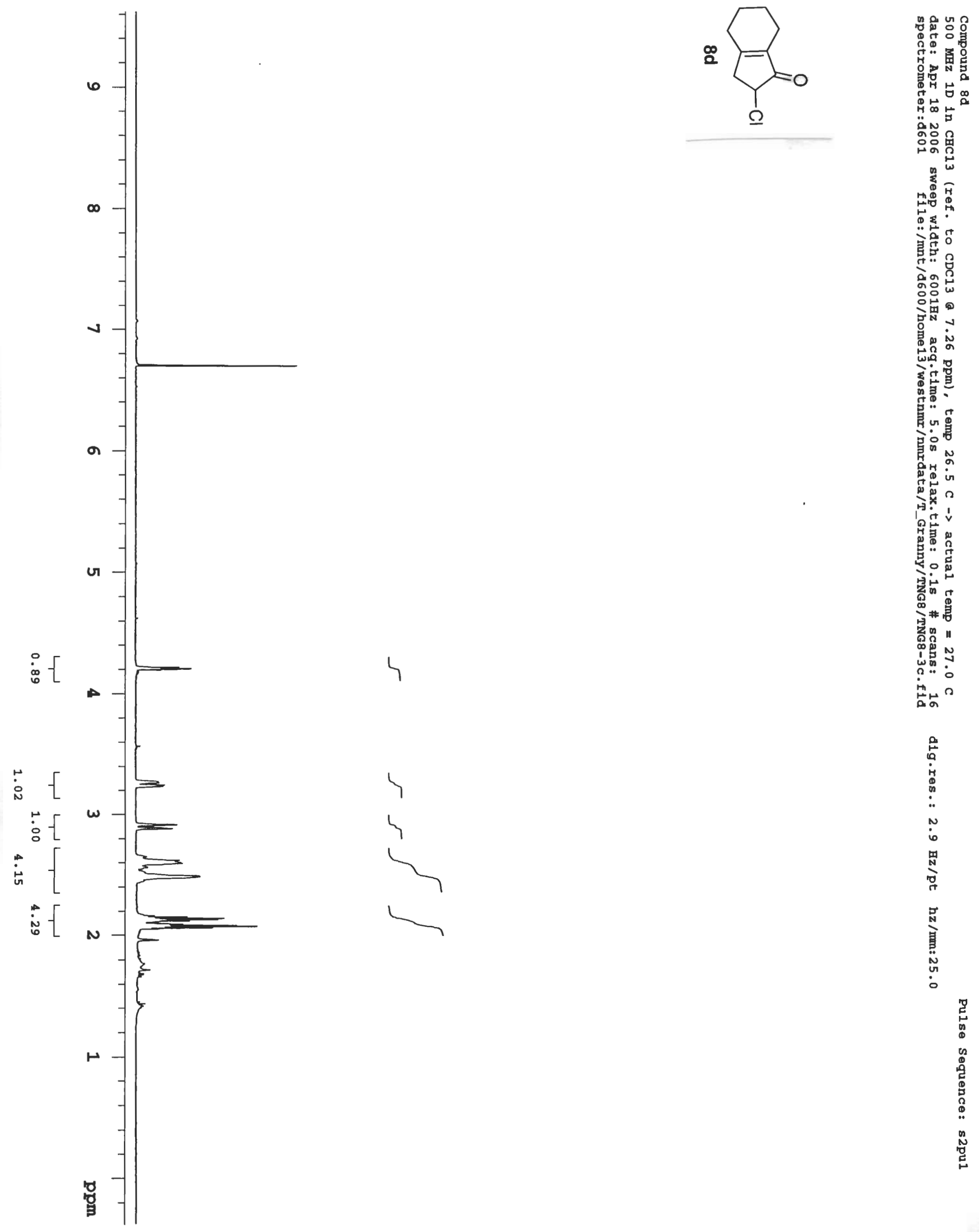

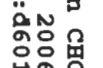

点

品密

" ?

最

令官。

เै.

ㅇํㅇํํ

它

最祭

证

o

当“行

这

हू

要点 in

कै

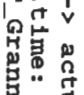

昰。范

荌占

音

苔骂"

政

م.

密台

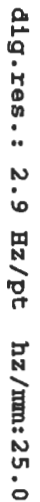

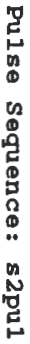




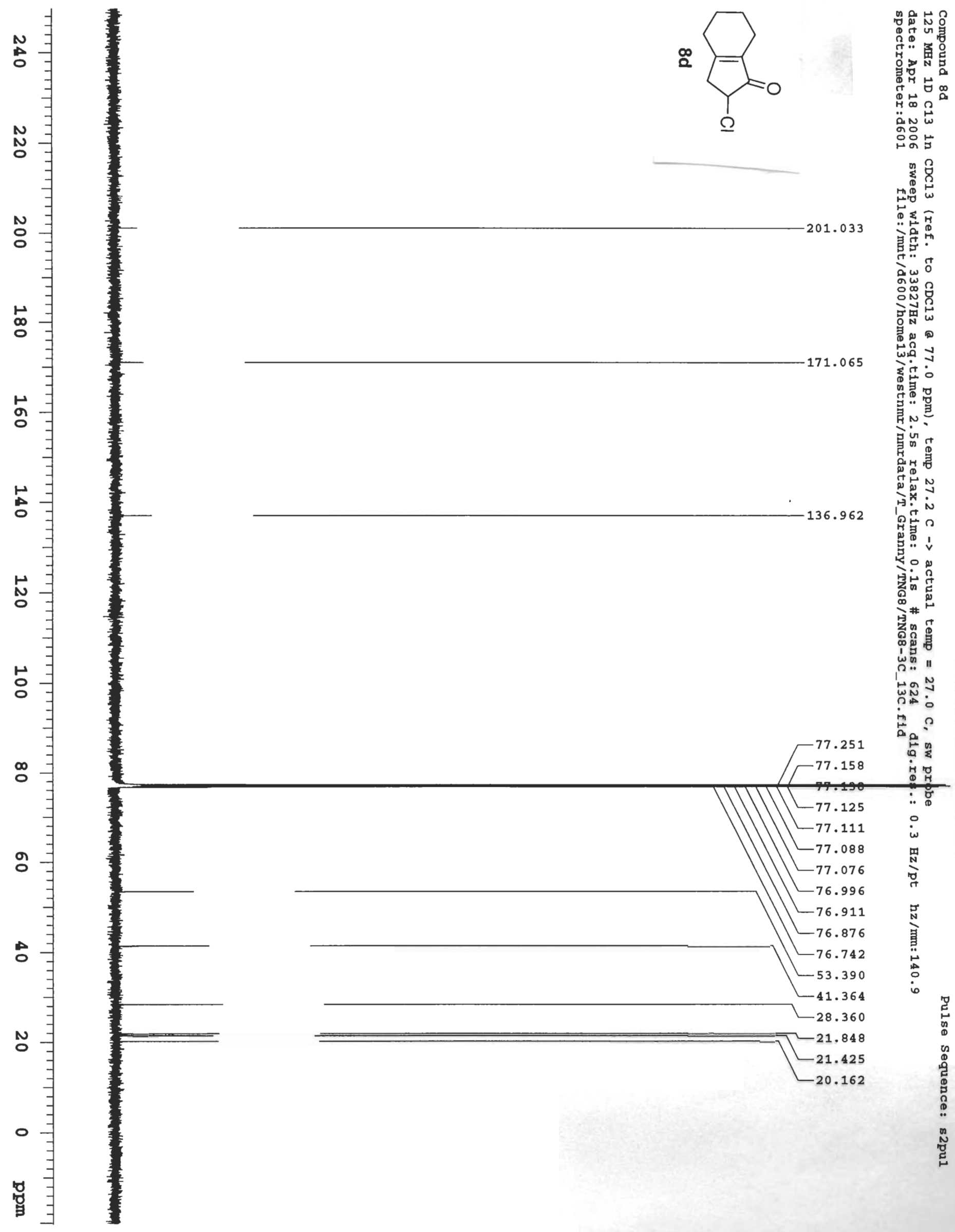




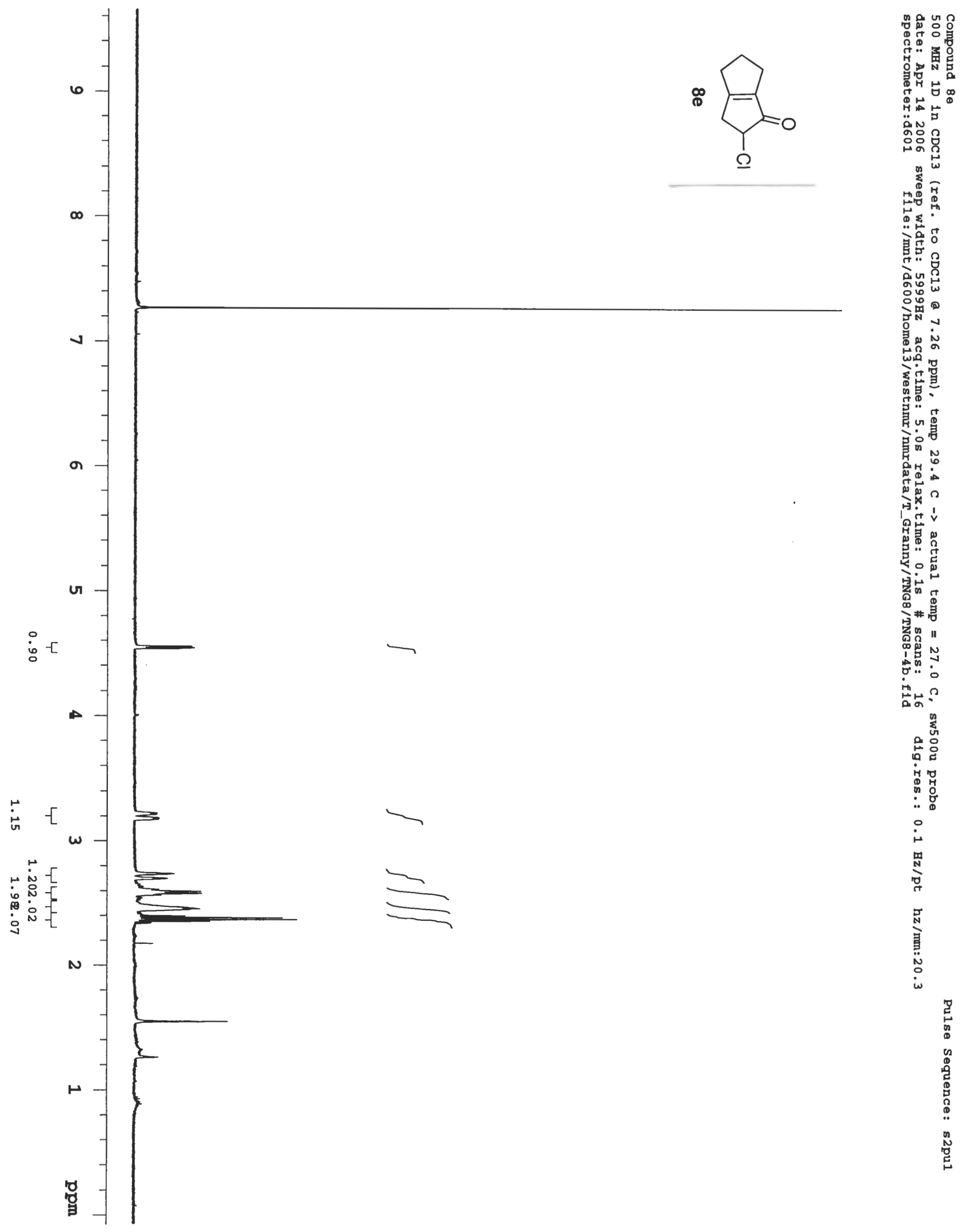




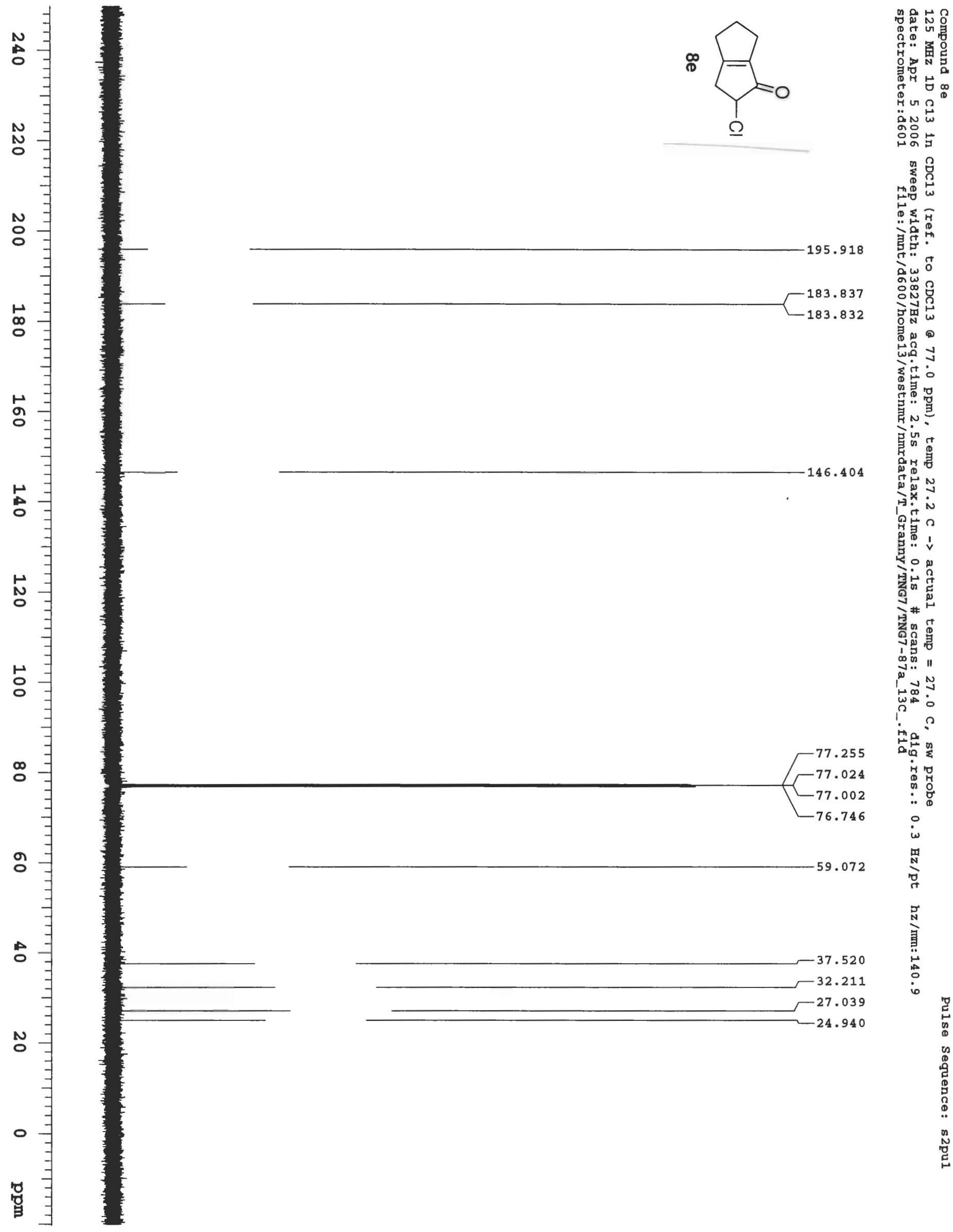




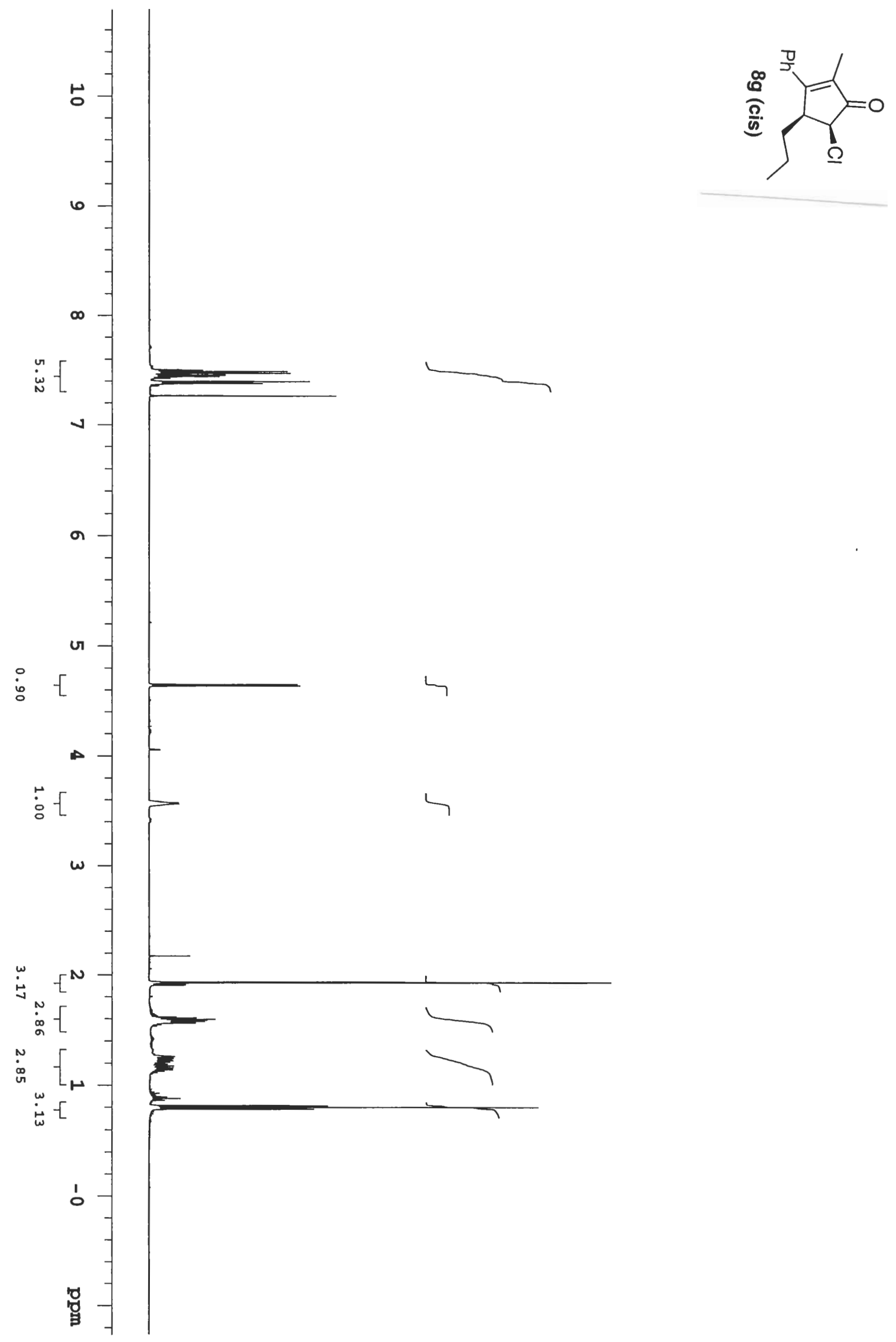

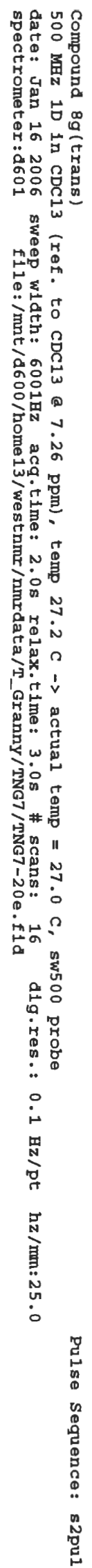




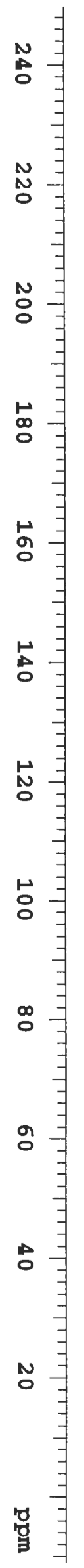

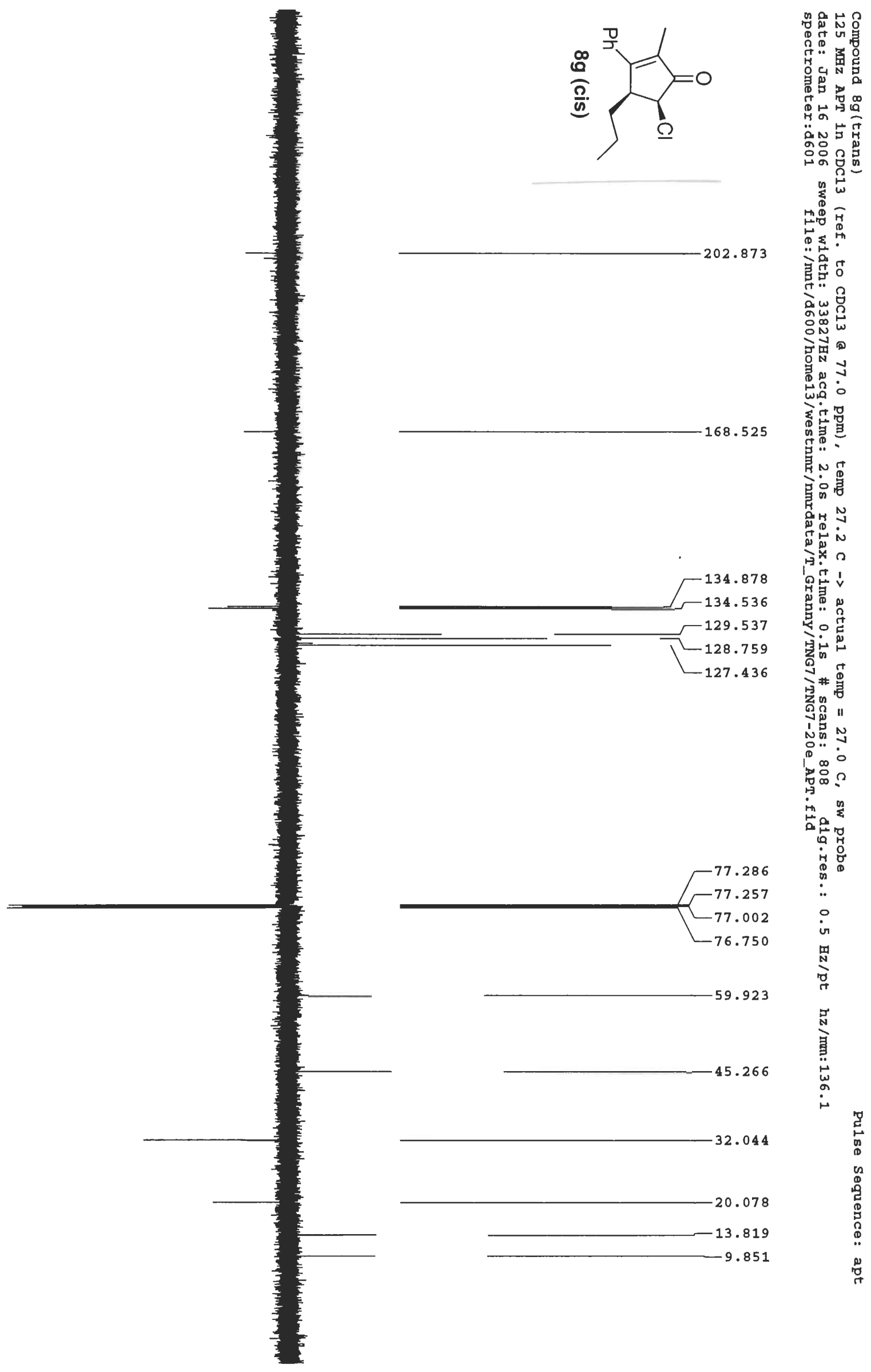



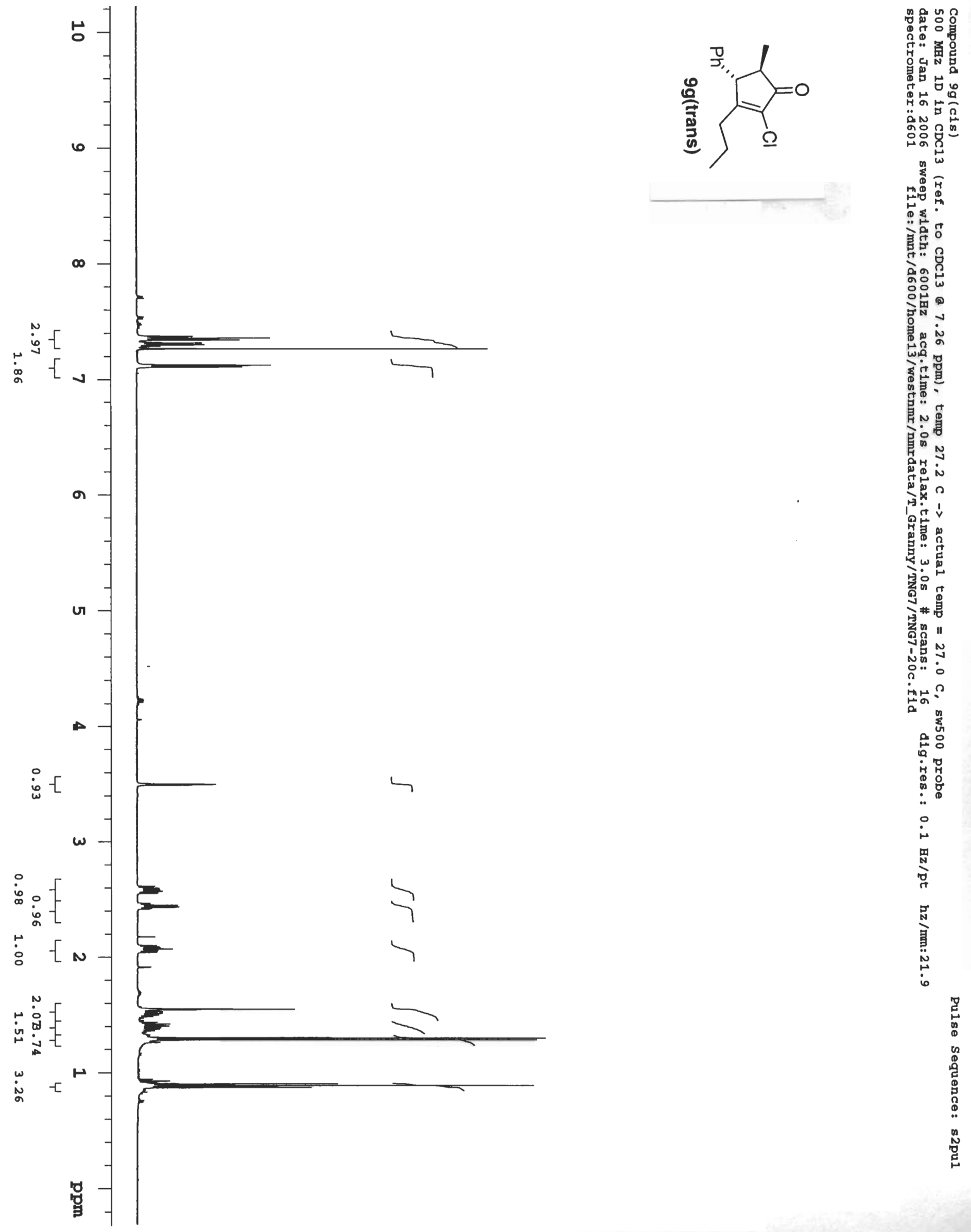

웅

突要

ํํㄹ

品

宽 ه

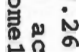

के

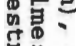

鼠”

思

然出

po

is

㟧

w

宓告

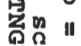

일

ㅇ..잉

int?

它

운응

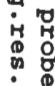

$\therefore$

i

突

兽 

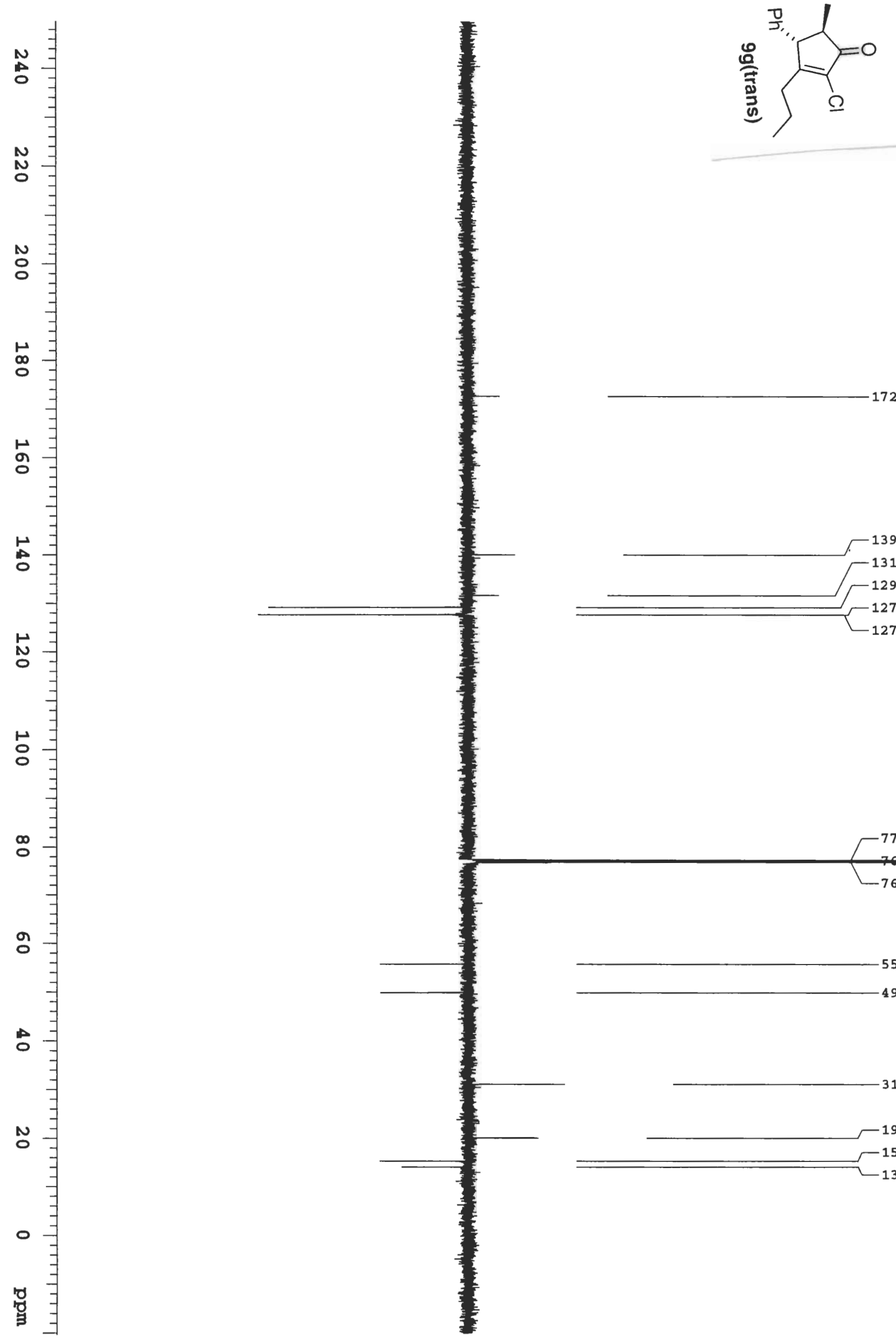

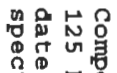

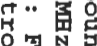

寻嵒。

苗的曷

品合

S요

员

ํㅜㄹ

궁

DE?

官营哭

त.

응

思

곰

思

空

$-172.687$

的

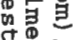

灵心

它总

管

\%

赵

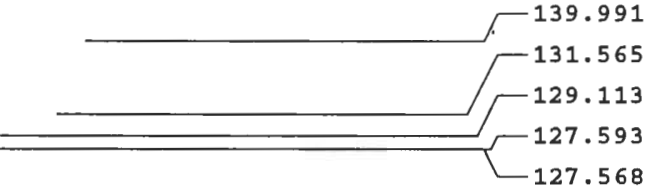

角。

官它

照昜

㐘。哭

晏占

宇\#苋

ดู่

占㫛 N

مि

管?

空。

in

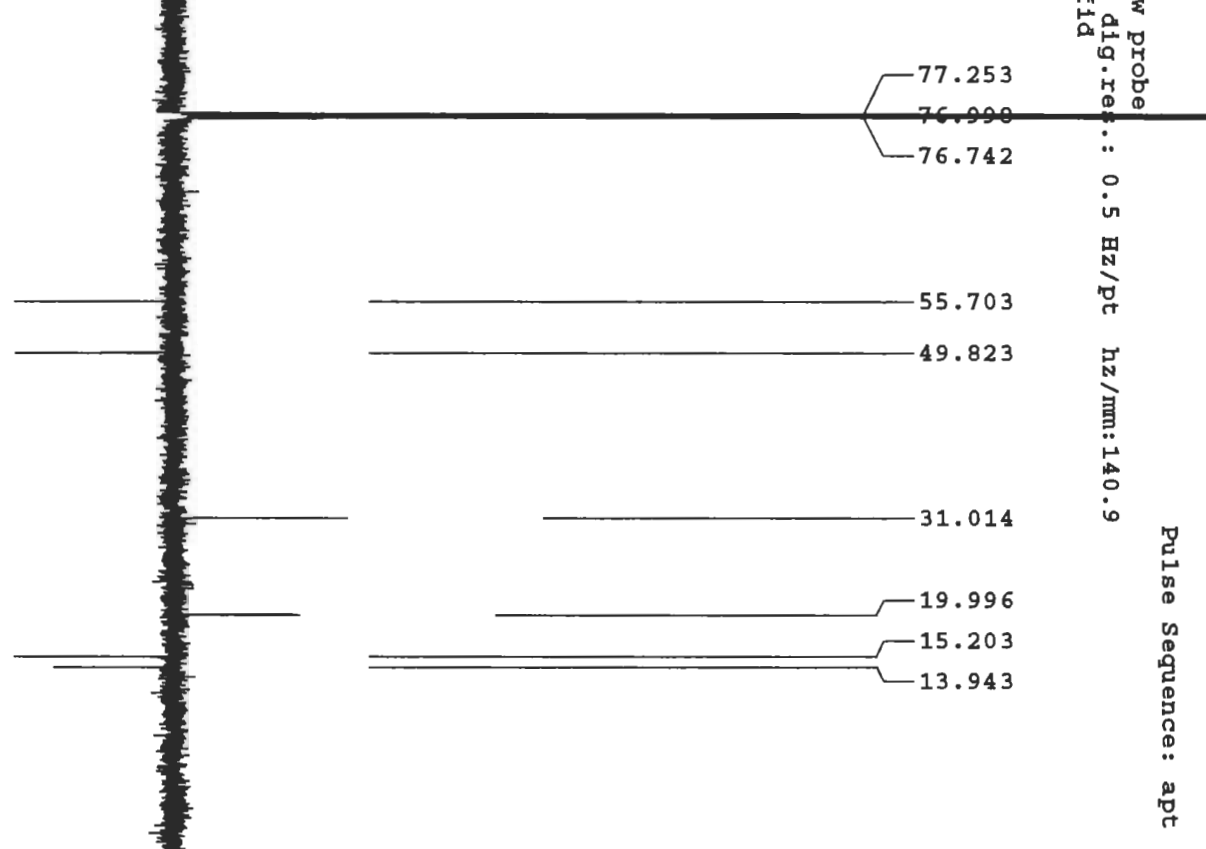




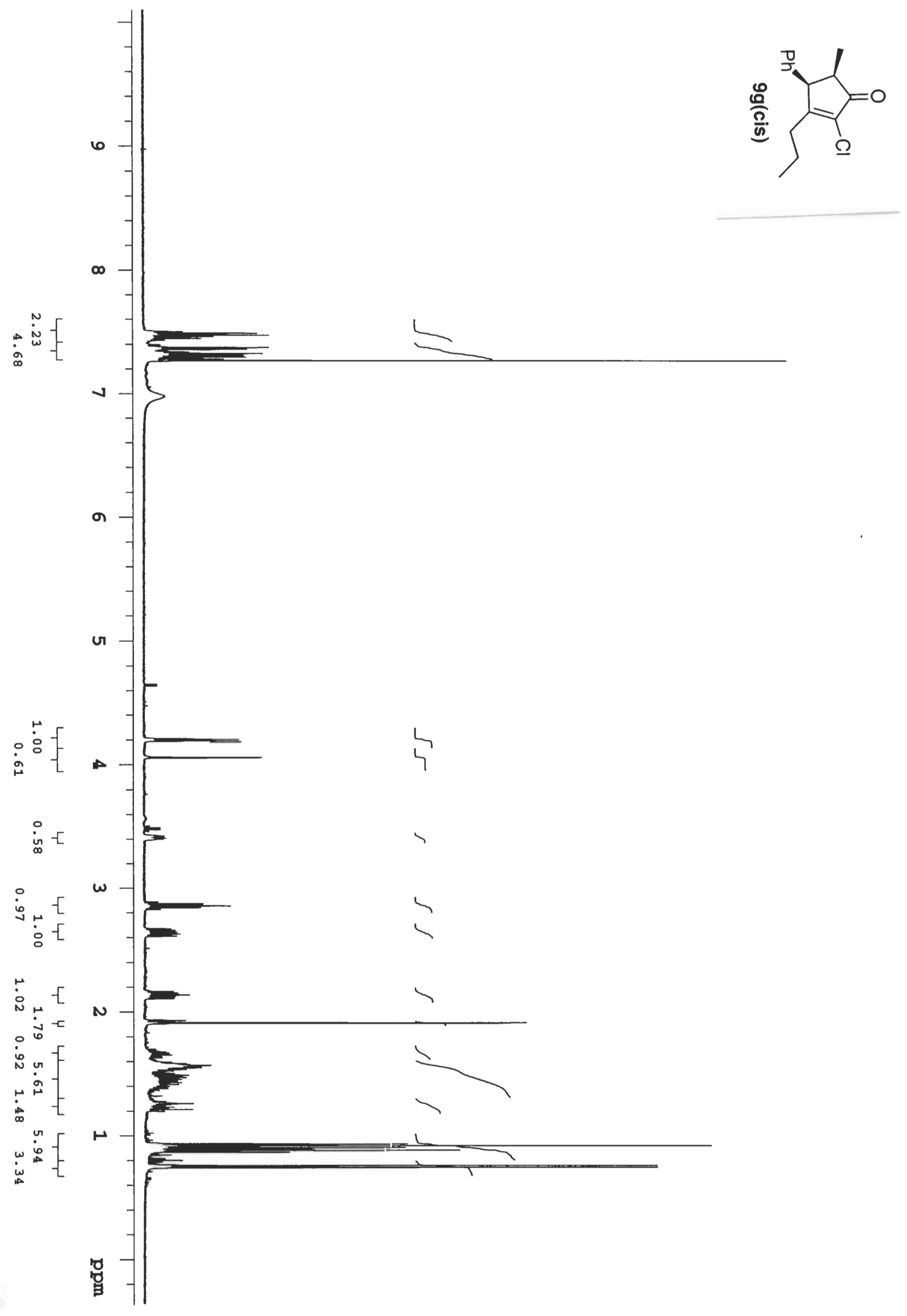




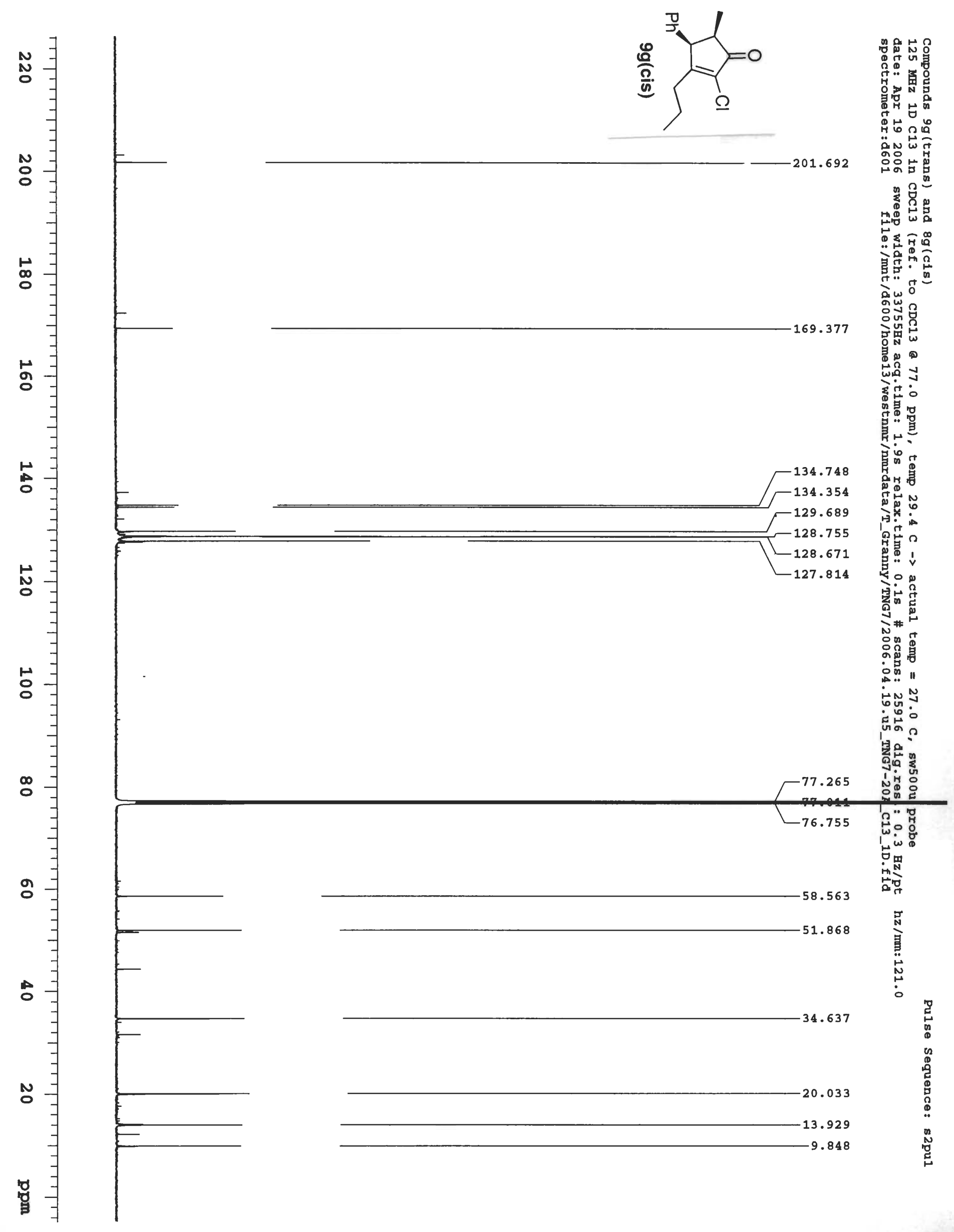

